\title{
MESURES D'INDÉPENDANCE LINÉAIRE DE LOGARITHMES DANS UN GROUPE ALGÉBRIQUE COMMUTATIF
}

\author{
ÉRIC GAUDRON
}

\begin{abstract}
RÉSUMÉ. This work falls within the theory of linear forms in logarithms over a connected and commutative algebraic group, defined over the field of algebraic numbers $\overline{\mathbb{Q}}$. Let $G$ be such a group. Let $W$ be a hyperplane of the tangent space at the origin of $G$, defined over $\overline{\mathbb{Q}}$, and $u$ be a complex point of this tangent space, such that the image of $u$ by the exponential map of the Lie group $G(\mathbb{C})$ is an algebraic point. Then we obtain a lower bound for the distance between $u$ and $W \otimes \mathbb{C}$, which improves the results known before and which is, in particular, the best possible for the height of the hyperplane $W$. The proof rests on Baker's method and Hirata's reduction as well as a new arithmetic argument (Chudnovsky's process of variable change) which enables us to give a precise estimate of the ultrametric norms of some algebraic numbers built during the proof.
\end{abstract}

\section{INTRODUCTION}

L'objet de cet article est de démontrer deux nouveaux résultats, concernant la théorie des formes linéaires de logarithmes, que nous avions présentés (sous une forme très légèrement plus faible) dans [16].

La problématique est la suivante. Étant donné un groupe algébrique commutatif G défini sur $\overline{\mathbb{Q}}$, un hyperplan $W$ de l'espace tangent à l'origine de $\mathbf{G}$, également défini sur $\overline{\mathbb{Q}}$, et $\mathbf{u}$ un point complexe de cet espace tangent, d'exponentielle (de Lie) algébrique, il s'agit d'étudier la distance qui sépare $\mathbf{u}$ de cet hyperplan $W$.

Si u appartient à $W \otimes \mathbb{C}$, un théorème de Wüstholz [34] assure l'existence d'un sous-groupe $\widetilde{\mathbf{G}}$ de $\mathbf{G}$ tel que $\mathbf{u} \in t_{\widetilde{\mathbf{G}}}(\mathbb{C}) \subseteq W \otimes \mathbb{C}$. Nous redémontrons ce théorème en apportant une précision supplémentaire sur le groupe $\widetilde{\mathbf{G}}$ : son degré (relatif à un plongement fixé de $\mathbf{G}$ dans un espace projectif) admet un majorant effectif, indépendant de la hauteur de l'espace vectoriel $W$. Notons que le choix de cette hauteur n'a pas vraiment d'importance ici ; pour fixer les idées, l'on peut considérer la hauteur de $W$ au sens de W.M. Schmidt [27] après avoir sélectionné une base $($ sur $\overline{\mathbb{Q}})$ de l'espace tangent $t_{\mathbf{G}}$. L'information que l'on obtient sur le degré de $\mathbf{G}$ permet de quantifier la relation de dépendance algébrique entre les coordonnées de u. Par exemple, dans le cas particulier où $\mathbf{G}$ est une variété semi-abélienne, une conséquence du théorème que nous obtenons est le résultat suivant :

Théorème 1.1. Soit $\mathbf{G}$ une variété semi-abélienne définie sur $\overline{\mathbb{Q}}$, munie d'un plongement $\Phi$ dans un espace projectif. Notons exp l'exponentielle du groupe de Lie complexe $\mathbf{G}(\mathbb{C})$. Soient $W$ un hyperplan de l'espace tangent $t_{\mathbf{G}}$, défini sur $\overline{\mathbb{Q}}$, et $\mathbf{u} \in W \otimes \mathbb{C}$ tel que $\mathbf{p}:=\exp \mathbf{u} \in \mathbf{G}(\overline{\mathbb{Q}})$. Soient $h(\mathbf{p})$ la hauteur logarithmique absolue (cf. (1)) du point (projectif) $\Phi(\mathbf{p})$ et $\|$.$\| une norme hermitienne sur t_{\mathbf{G}} \otimes \mathbb{C}$. Soit $D$ un majorant du degré d'un corps de définition de $\mathbf{G}, W$ et $\mathbf{p}$. Soit $E$ un

\footnotetext{
${ }^{0}$ Institut Fourier. UMR 5582, BP 74, 38402 Saint-Martin-d'Hères Cedex France Eric.Gaudron@ujf-grenoble.fr

${ }^{0} \mathrm{MSC} 2000: 11 \mathrm{~J} 86,11 \mathrm{~J} 20(14 \mathrm{~L} 10)$
} 
nombre réel quelconque $\geq e$. Enfin, considérons $a \in \mathbb{R}$ tel que

$$
\log a \geq \max \left\{1, h(\mathbf{p}), \frac{(E\|\mathbf{u}\|)^{2}}{D}\right\} .
$$

Alors $i l$ existe une constante $c_{1}=c_{1}(\mathbf{G}, \Phi,\|$.$\| ) (en particulier indépendante des$ hauteurs de $\mathbf{p}$ et de $W$ ) et il existe un sous-groupe algébrique connexe $\widetilde{\mathbf{G}}$ de $\mathbf{G}$, de dimension $\widetilde{d}$, vérifiant les conditions suivantes :

(1) $\mathbf{u} \in t_{\widetilde{\mathbf{G}}}(\mathbb{C}) \subseteq W \otimes \mathbb{C}$.

(2) Le degré $\operatorname{deg}_{\Phi} \widetilde{\mathbf{G}}$ de $\widetilde{\mathbf{G}}$, relatif au plongement $\Phi$, est majoré par

$$
c_{1} D \max \left\{1, \frac{D \log a}{\log E}\right\}^{\widetilde{d}} \times \max \left\{1, \frac{D}{\log E} \log ^{+}\left(\frac{D \log a}{\log E}\right)\right\}^{\widetilde{d}+1} .
$$

Ce type d'énoncé est rarement mis en relief en dépit de la (relative) simplicité avec laquelle il se déduit de la démonstration d'une mesure d'indépendance linéaire de logarithmes. Dans [12], p. 10, S. DAVID fournit une majoration du degré de $\widetilde{\mathbf{G}}$, dans le cas particulier où $\mathbf{G}$ est un produit de courbes elliptiques, avec une constante $c_{1}$ explicite (mais sa borne dépend de la hauteur de $W$ ).

Lorsque $\mathbf{u} \notin W \otimes \mathbb{C}$, nous obtenons une minoration de la distance $\mathrm{d}(\mathbf{u}, W \otimes \mathbb{C})$, optimale en la hauteur $h(W)$ de l'espace $W$ :

Théorème 1.2. Soit $\mathbf{G}$ un groupe algébrique commutatif, défini sur $\overline{\mathbb{Q}}$. Soit $W$ un hyperplan de l'espace tangent à l'origine $t_{\mathbf{G}}$, défini sur un corps de nombres de degré $D$, et soit $\mathbf{u} \in t_{\mathbf{G}}(\mathbb{C})$ tel que $\exp \mathbf{u} \in \mathbf{G}(\overline{\mathbb{Q}})$. Munissons $t_{\mathbf{G}}(\mathbb{C})$ d'une distance hermitienne $\mathrm{d}$. Il existe une constante $c_{2}=c_{2}(\mathbf{G}, \mathrm{d}, \mathbf{u}, D)$ (indépendante de la hauteur de $W$ ) telle que, si $\mathbf{u} \notin W \otimes \mathbb{C}$, alors

$$
\log \mathrm{d}(\mathbf{u}, W \otimes \mathbb{C}) \geq-c_{2} \max \{1, h(W)\} .
$$

Ce théorème améliore la dépendance en $h(W)$ du résultat antérieur de N. HiRATAKoHno [20], qui comportait une puissance de $\log h(W)$ supplémentaire. De ce point de vue, nous généralisons également les travaux récents de M. ABLY [1], S. DAVID $\&$ N. Hirata-Kohno [13, qui ne traitaient que le cas particulier (ou un sous-cas) du produit d'un groupe affine $\mathbb{G}_{\mathrm{a}}$ par des courbes elliptiques. Pour un historique détaillé des progrès réalisés sur cette question, nous renvoyons le lecteur à 113. Signalons que ces deux derniers auteurs ont obtenu récemment un résultat de ce type, toujours dans le cas particulier ci-dessus, lorsque $W$ est de codimension quelconque $\geq 1$ dans $t_{\mathbf{G}}$.

Comme me l'a fait remarquer le rapporteur, il est important de noter que ces deux théorèmes se complètent mutuellement en fournissant une version plus fine du premier. En effet, il est possible de les lire d'une manière légèrement différente, à l'instar du théorème 2.1 de S. DAVID dans [12]. Pour aboutir à la conclusion du théorème 1.1 ci-dessus, nous avons supposé que le logarithme de $\mathbf{u}$ appartenait à l'espace $W \otimes \mathbb{C}$. En réalité, il suffit, pour obtenir cette même conclusion, de supposer que la distance de $\mathbf{u}$ à $W \otimes \mathbb{C}$ est ii petite $i \dot{\iota}$, la quantification précise de cette petitesse étant fournie par le théorème 1.2, puisque, par contraposée, si $\log \mathrm{d}(\mathbf{u}, W \otimes \mathbb{C})<-c_{2} \max \{1, h(W)\}$ alors $u \in W \otimes \mathbb{C}$ et le théorème 1.1 s'applique. Cette observation vaut également pour le théorème 3.1 qui va suivre.

Dans le cadre général d'un groupe algébrique commutatif, toutes les mesures d'indépendance linéaire de logarithmes, obtenues jusqu'à maintenant, ont leur schéma de démonstration fondé sur l'article original de P. PHILIPpon \& M. WALDsCHMidT [25], avec en toile de fond la méthode de Baker. En 1991, N. HiRATAKoнno introduisit une nouvelle idée qui permit d'améliorer considérablement la dépendance de la mesure en le paramètre ii hauteur de l'hyperplan i.i. Cette idée consiste à modifier légèrement le groupe algébrique et l'hyperplan de départ, puis à se servir du facteur $\mathbb{G}_{\mathrm{a}}$ introduit pour juguler l'influence des dérivations. Le nouvel 
argument qui optimise la mesure pour ce paramètre (théorème 1.2 ci-dessus) est inspiré des travaux de D.V. \& G.V. Chudnovsky [9, 10, 11], et il a été mis en ouvre pour la première fois dans ce contexte par S. David \& N. Hirata-Kohno [13, 14]. Il repose sur un changement de variable qui donne lieu, pour la partie arithmétique de la preuve, à un raisonnement direct avec les paramètres locaux du groupe algébrique et non, comme auparavant, avec ceux de l'espace tangent du groupe. Cela revient à utiliser le logarithme (formel) en lieu et place de l'exponentielle (ce qui, au fond, pour obtenir des formes linéaires de logarithmes peut sembler être une idée bien naturelle) pour les estimations des normes ultramétriques des nombres algébriques qui apparaissent au cours de notre démonstration. C'est donc la méthode générale de [25], précédée d'un conditionnement des données (groupe algébrique, hyperplan) et à laquelle s'ajoute le choix pertinent des variables locales (selon la nature des estimations à effectuer), qui permet d'obtenir les résultats du paragraphe 3.1 et d'en déduire en particulier les théorèmes 1.1 et 1.2 .

Notations et conventions. Si $x$ est un nombre réel, $[x]\left(\right.$ resp. $\left.x^{+}\right)$désigne la partie entière de $x$ (resp. le maximum de $x$ et 0 ). De même, card désigne le cardinal d'un ensemble et Vol le volume d'un objet. Dans ce texte, $\ln =\log$ est le logarithme népérien (éventuellement étendu à un ouvert simplement connexe de $\mathbb{C} \backslash\{0\}$ ). Et $\log ^{+}$désigne la fonction d'une variable réelle $x \mapsto \log \max \{1, x\}$.

Soit $\mathbf{K}$ un corps de nombres, d'anneau des entiers $\mathcal{O}_{\mathbf{K}}$. Nous désignons par $M_{\mathbf{K}}$ l'ensemble des places de $\mathbf{K}$. Pour $v \in M_{\mathbf{K}}$, la valeur absolue $|\cdot|_{v}$ sur le complété $\mathbf{K}_{v}$ est normalisée de la manière suivante :

$$
\begin{cases}|1|_{v}=1 & \text { si } v \text { est archimédienne, } \\ |p|_{v}=\frac{1}{p} & \text { si } v \text { est ultramétrique, au-dessus du nombre premier } p .\end{cases}
$$

Si $\mathbf{x}=\left(x_{0}: \cdots: x_{g}\right) \in \mathbb{P}^{g}(\mathbf{K})$, le réel $h(\mathbf{x})$ est la hauteur de Weil logarithmique absolue de $\mathbf{x}$ (voir par exemple [33], p. 77) :

$$
h(\mathbf{x}):=\frac{1}{[\mathbf{K}: \mathbb{Q}]} \sum_{v \in M_{\mathbf{K}}}\left[\mathbf{K}_{v}: \mathbb{Q}_{v}\right] \log \max \left\{\left|x_{0}\right|_{v}, \ldots,\left|x_{g}\right|_{v}\right\} .
$$

Lorsque $a_{1}, \ldots, a_{h} \in \overline{\mathbb{Q}}$ sont les coefficients d'un polynôme $P$, nous noterons $h(P)$ la hauteur du point projectif $\left(1: a_{1}: \cdots: a_{h}\right)$. Si $\alpha \in \overline{\mathbb{Q}}$, l'écriture $|\bar{\alpha}|$ désigne le maximum des valeurs absolues des conjugués de $\alpha$.

Si $\mathbf{G}$ est un groupe algébrique complexe, $\exp _{\mathbf{G}}: t_{\mathbf{G}} \rightarrow \mathbf{G}$ désigne l'application exponentielle de $\mathbf{G}$ et $\Omega_{\mathbf{G}}$ le noyau de cette application (sous-groupe des périodes). Nous aurons à considérer l'exponentielle normalisée au sens de [28], ce que nous détaillerons plus loin au $\S$ 5.

Si $V=V_{1} \times \cdots \times V_{n}$ est une variété sur un corps $\mathbf{K}$, on désigne par $\mathscr{H}\left(V ; X_{1}, \ldots, X_{n}\right)$ la partie homogène de plus haut degré du polynôme de Hilbert-Samuel multihomogène de $V$, multipliée par $(\operatorname{dim} V)$ ! (voir 23 ).

$\mathrm{Au}$ cours de la démonstration des théorèmes du $\S 3.1$ apparaissent, en nombre fini, des constantes $c$, indexées par $\mathbb{N}$, qui ne dépendent que du groupe algébrique $\mathbf{G}$, d'un éventuel plongement de ce groupe dans un espace projectif, d'une base algébrique de l'espace tangent à $\mathbf{G}$ et de la distance choisie sur $t_{\mathbf{G}}(\mathbb{C})$. Par convention et par commodité, ces constantes seront positives et choisies de manière croissante en fonction de leur indice $\left(c_{1} \leq c_{2} \leq \cdots\right)$. Elles seront toutes plus petites que le nombre réel $C_{0}$ introduit lors du choix des paramètres, au $\S 6.2$.

\section{DonnÉES}

2.1. Le groupe algébrique. Soient $n$ un entier naturel non nul et $\mathbf{K}$ un corps de nombres plongé dans $\mathbb{C}$ (nous noterons parfois $\sigma_{0}: \mathbf{K} \rightarrow \mathbb{C}$ le plongement choisi de 
$\mathbf{K}$ dans $\mathbb{C}$ ). Considérons $\mathbf{G}_{1}, \ldots, \mathbf{G}_{n}$ des groupes algébriques commutatifs, définis sur K. Notons

$$
\mathbf{G}_{0}:=\mathbb{G}_{\mathrm{a}}, \quad \mathbf{G}:=\mathbf{G}_{0} \times \mathbf{G}_{1} \times \cdots \times \mathbf{G}_{n}
$$

le groupe algébrique produit, $\delta_{\ell}$ la dimension de $\mathbf{G}_{\ell}$ et $d+1:=\delta_{0}+\delta_{1}+\ldots+\delta_{n}$ celle de $\mathbf{G}$. Soit $\mathbf{e}:=\left(e_{m}\right)_{0 \leq m \leq d}$ une base du $\mathbf{K}$-espace tangent (à l'origine) $t_{\mathbf{G}}$ telle que

$$
\mathbf{e}_{i}=\left(e_{\delta_{0}+\cdots+\delta_{i-1}}, \ldots, e_{\delta_{0}+\cdots+\delta_{i}-1}\right)
$$

forme une base de $t_{\mathbf{G}_{i}}$ pour $i \in\{0, \ldots, n\}$ (par convention d'écriture, $\delta_{-1}:=-1$ ). Nous choisissons un plongement admissible ${ }^{1} \Phi$ du groupe $\mathbf{G}$ dans un espace multiprojectif $\mathbb{P}:=\mathbb{P}^{N_{0}} \times \cdots \times \mathbb{P}^{N_{n}}$, défini sur $\mathbf{K}$, et nous noterons $\rho_{j}$ un majorant $\geq 1$ de l'ordre ${ }^{2}$ d'une représentation de l'exponentielle du groupe de Lie $\mathbf{G}_{j}(\mathbb{C})$ dans ce plongement. Dans la pratique, nous prendrons $\rho_{j}=1$ si $\mathbf{G}_{j}$ est un groupe linéaire (commutatif) et $\rho_{j}=2$ si $\mathbf{G}_{j}$ a une partie abélienne non triviale. Ces objets possèdent quelques propriétés supplémentaires (en particulier d'intégralité) que nous développons au cours de la démonstration du lemme 6.33.

2.2. Données arithmétiques. Soient $\mathbf{L}$ un corps de nombres contenant $\mathbf{K}, D$ l'entier naturel $[\mathbf{L}: \mathbb{Q}]$ et $\mathbf{p} \in \mathbf{G}(\mathbf{L})$. Soit $\xi_{1} \ldots, \xi_{D}$ une base quelconque du $\mathbb{Q}$ espace vectoriel $\mathbf{L}$. Le réel $h\left(\xi_{1}: \cdots: \xi_{D}\right)$ est la hauteur logarithmique absolue de $\left(\xi_{1}: \cdots: \xi_{D}\right) \in \mathbb{P}^{D-1}(\mathbf{L})$. Nous supposerons que $\mathbf{L}$ est plongé dans $\mathbb{C}$ via un prolongement de $\sigma_{0}$ (que nous noterons encore $\sigma_{0}$ ).

Considérons $\mathbf{u}=u_{0} e_{0}+\cdots+u_{d} e_{d} \in t_{\mathbf{G}}(\mathbb{C})$ un logarithme de $\mathbf{p}$, i.e. $\exp _{\mathbf{G}(\mathbb{C})}(\mathbf{u})=$ $\mathbf{p}$ (en particulier $u_{0} \in \mathbf{L}$ ). Soit $\mathbf{p}_{j}$ l'image de $\mathbf{p}$ dans $\mathbf{G}_{j}$ et $\mathbf{u}_{j}$ un logarithme de $\mathbf{p}_{j}$ tel que $\mathbf{u}=\left(\mathbf{u}_{0}, \ldots, \mathbf{u}_{n}\right)$. Le réel $h\left(\mathbf{p}_{j}\right)$ est la hauteur (logarithmique absolue) du point algébrique $\Phi\left(\mathbf{p}_{j}\right)$ dans l'espace projectif $\mathbb{P}^{N_{j}}$. Lorsque $n$ est un entier naturel, nous notons

$$
\Gamma_{\mathbf{p}}(n):=\{0, \mathbf{p}, \ldots, n \mathbf{p}\} .
$$

Enfin soit $W$ un hyperplan de $t_{\mathbf{G}} \otimes_{\mathbf{K}} \mathbf{L}$ d'équation

$$
z_{0}=\beta_{1} z_{1}+\cdots+\beta_{d} z_{d}
$$

dans la base e, où $\beta_{1}, \ldots, \beta_{d}$ sont des éléments de $\mathbf{L}$. Ici la hauteur $h(W)$ de $W$ est la hauteur logarithmique absolue de $\left(1: \beta_{1}: \cdots: \beta_{d}\right)$. Ainsi définie, cette hauteur dépend du choix de la base e. À une fonction bornée près, c'est la hauteur définie par W.M. Schmidt [27] et aussi celle définie par J.-B. Bost dans [7], p. 133.

Posons également $\Lambda:=u_{0}-\beta_{1} u_{1}-\cdots-\beta_{d} u_{d}$. Le théorème porte sur une minoration de la distance de $\mathbf{u}$ à $W$ lorsque cette quantité n'est pas nulle. Pour énoncer le théorème du paragraphe suivant, nous fixons une norme hermitienne $\|$. sur $t_{\mathbf{G}}(\mathbb{C})$ et nous notons d la distance associée à cette norme. Par exemple, si nous choisissons la norme qui rend la base e orthonormée, un calcul immédiat (à l'aide des déterminants de Gram par exemple) fournit l'égalité

$$
\mathrm{d}(\mathbf{u}, W)=\frac{|\Lambda|}{\left(1+\left|\beta_{1}\right|^{2}+\cdots+\left|\beta_{d}\right|^{2}\right)^{1 / 2}} .
$$

Ainsi, il est équivalent de minorer $\mathrm{d}(\mathbf{u}, W)$ et de minorer le module de la forme linéaire $\Lambda$, si l'on ne tient pas compte de la dépendance en la distance d. Pour la démonstration, la norme $\|$.$\| sera l'unique norme qui rend isométrique l'isomorphis-$ me $t_{\mathbf{G}}(\mathbb{C}) \simeq \mathbb{C}^{d+1}$, fixé par la base e (l'espace $\mathbb{C}^{d+1}$ étant muni de son produit

\footnotetext{
${ }^{1} \mathrm{Au}$ sens du $\S$ 5. On dit aussi parfois plongement ii à la Serre ¿¿, en référence à l'appendice 28 de 29], écrit par J.-P. SERRE sur ce sujet.

${ }^{2}$ Lorsque $f\left(z_{1}, \ldots, z_{\ell}\right)$ est une fonction analytique à $\ell$ variables, l'ordre de $f$ est par définition$$
\limsup _{R \rightarrow \infty} \frac{\log \log ^{+} \max _{\left|z_{i}\right| \leq R}\left|f\left(z_{1}, \ldots, z_{\ell}\right)\right|}{\log R} .
$$ 
hermitien canonique). Mais pour l'énoncé des résultats au paragraphe suivant, la distance d et la base e seront dissociées et quelconques.

\section{RÉsultats}

3.1. Énoncés. Le résultat principal de cet article est le suivant.

Théorème 3.1. Il existe une constante effective $c_{3}>0$, ne dépendant que du triplet $(\mathbf{G}, \Phi, \mathbf{e}, \mathrm{d})$, ayant la propriété suivante. Soit $E$ un réel $\geq e$. Posons

$$
\mathfrak{a}:=1+\left[\frac{D}{\log E} \log \left(1+\frac{D+\sum_{j=1}^{n}\left(h\left(\mathbf{p}_{j}\right)+\left(E\left\|\mathbf{u}_{j}\right\|\right)^{\rho_{j}}\right)}{\log E}\right)\right]
$$

et

$$
\begin{aligned}
U:= & \frac{\operatorname{card}\left(\Gamma_{\mathbf{p}}(\mathfrak{a})\right)}{(\mathfrak{a} \log E)^{d}} \times\left(D h(W)+D h\left(u_{0}\right)+\mathfrak{a} \log E\right) \\
& \times \prod_{j=1}^{n}\left(\mathfrak{a} \log E+D \max _{0 \leq s \leq c_{3} \mathfrak{a}}\left\{h\left(s \mathbf{p}_{j}\right)\right\}+\left(E \mathfrak{a}\left\|\mathbf{u}_{j}\right\|\right)^{\rho_{j}}\right)^{\delta_{j}} .
\end{aligned}
$$

- $S i \mathbf{u} \in W_{\mathbb{C}}$, alors il existe un sous-groupe algébrique connexe $\widetilde{\mathbf{G}}$ de $\mathbf{G}_{\mathbf{L}}$, de dimension $\widetilde{d}$, tel que

(a) L'espace tangent à l'origine $t_{\widetilde{\mathbf{G}}}$ est inclus dans l'hyperplan $W$.

(b) Le point $\mathbf{u}$ appartient à $t_{\widetilde{\mathbf{G}}}(\mathbb{C})$.

(c) Si $\pi: \mathbf{G} \longrightarrow \prod_{j=1}^{n} \mathbf{G}_{j}$ désigne la projection canonique, il existe un entier $m \geq 1$ et des entiers distincts $1 \leq j_{1}<\cdots<j_{m} \leq n$ tels que le degré de $\pi(\widetilde{\mathbf{G}})$ (relatif au plongement choisi de $\mathbf{G}$ dans un espace projectif) est majoré par

$$
\begin{aligned}
& c_{3} \frac{\operatorname{card}\left(\Gamma_{\mathbf{p}}(\mathfrak{a})\right)}{(\mathfrak{a} \log E)^{\tilde{d}}} \times \prod_{\ell=1}^{m-1}\left(\mathfrak{a} \log E+D \max _{0 \leq s \leq c_{3} \mathfrak{a}}\left\{h\left(s \mathbf{p}_{j_{\ell}}\right)\right\}+\left(E \mathfrak{a}\left\|\mathbf{u}_{j_{\ell}}\right\|\right)^{\rho_{j_{\ell}}}\right)^{\delta_{j_{\ell}}} \\
& \times\left(\mathfrak{a} \log E+D \max _{0 \leq s \leq c_{3} \mathfrak{a}}\left\{h\left(s \mathbf{p}_{j_{m}}\right)\right\}+\left(E \mathfrak{a}\left\|\mathbf{u}_{j_{m}}\right\|\right)^{\rho_{j_{m}}}\right)^{\widetilde{d}-\left(\delta_{j_{1}}+\cdots+\delta_{j_{m-1}}\right)} \\
& \times \max \left\{1, \frac{D h\left(\xi_{1}: \cdots: \xi_{D}\right)+D \log (D)}{U}\right\} .
\end{aligned}
$$

- Si $\mathbf{u} \notin W_{\mathbb{C}}$, alors :

$$
\log \mathrm{d}(\mathbf{u}, W) \geq-c_{3} \max \left\{U, D h\left(\xi_{1}: \cdots: \xi_{D}\right)+D \log (D)\right\} .
$$

Dans le cas particulier où la forme linéaire est non-homogène, nous avons le résultat suivant plus précis.

Théorème 3.2. Avec les notations du théorème 3.1, supposons que le nombre complexe $u_{0}$ est non nul et que le groupe algébrique $\prod_{j=1}^{n} \mathbf{G}_{j}$ est une variété semiabélienne. Alors u n'appartient pas à $W$ et il existe une constante $c_{4}>0$, ne dépendant que du triplet $(\mathbf{G}, \Phi, \mathbf{e}, \mathrm{d})$, telle que, si

$$
\begin{aligned}
U^{\prime}:= & \frac{\mathfrak{a}}{(\mathfrak{a} \log E)^{d}} \times\left(D h(W)+D \max _{0 \leq s \leq c_{4} \mathfrak{a}}\left\{h\left(s u_{0}\right)\right\}+\log E\right) \\
& \times \prod_{j=1}^{n}\left(\mathfrak{a} \log E+D \max _{0 \leq s \leq c_{4} \mathfrak{a}}\left\{h\left(s \mathbf{p}_{j}\right)\right\}+\left(E \mathfrak{a}\left\|\mathbf{u}_{j}\right\|\right)^{\rho_{j}}\right)^{\delta_{j}}
\end{aligned}
$$

alors

$$
\log \mathrm{d}(\mathbf{u}, W) \geq-c_{4} \max \left\{U^{\prime}, D h\left(\xi_{1}: \cdots: \xi_{D}\right)+D \log (D)\right\} .
$$

La différence entre $U$ et $U^{\prime}$ réside dans le remplacement de la quantité $D h\left(u_{0}\right)+$ $\mathfrak{a} \log E$ par la quantité (plus petite) $D \max _{0 \leq s \leq c_{4} \mathfrak{a}}\left\{h\left(s u_{0}\right)\right\}+\log E$ (qui est de l'ordre de $\left.D h\left(u_{0}\right)+D \log \mathfrak{a}+\log E\right)$ de $U^{\prime}$. 
3.2. Commentaires et conséquences. Ce théorème contient les mesures d'indépendance linéaires de logarithmes obtenues par N. Hirata-KoHno [19] et S. DAVID [12], à l'exception, dans ce dernier cas, de la constante $c_{4}$ qui ici n'est pas totalement explicite. Il généralise également le résultat de S. DAVID \& N. HIRATAKoHno présenté dans 13. Toutefois, mentionnons que dans le cas particulier du produit d'une puissance du groupe affine $\mathbb{G}_{\mathrm{a}}$ par des courbes elliptiques, ces auteurs ont démontré une version ii simultanée ¿¿i où l'espace $W$ est de codimension quelconque $\geq 1$ [14. L'amélioration essentielle qu'apporte le théorème 3.1 par rapport à ces résultats est la disparition de termes parasites en $\log h(W)$, pour garder un minorant de $\log \mathrm{d}(\mathbf{u}, W)$ linéaire en $h(W)$ (la quantité $D h\left(\xi_{1}: \cdots \xi_{D}\right)$ pouvant être majorée par une fonction linéaire de $h(W)$, voir ci-dessous). Comme annoncée dans le théorème 1.2, la majoration $\log |\Lambda| \geq-c_{5} \max \{1, h(W)\}$ est alors optimale. En effet, supposons qu'il existe une fonction $b: \mathbb{R}^{+} \rightarrow \mathbb{R}^{+}$telle que $b(x) / x \underset{x \rightarrow \infty}{\longrightarrow} 0$ et telle que

$$
\log |\Lambda| \geq-b\left(h\left(1: u_{0}: \beta_{1}: \cdots: \beta_{d}\right)\right) .
$$

Alors le passage de $\left(u_{0}, \beta_{1}, \ldots, \beta_{d}\right)$ à $\left(\frac{u_{0}}{N}, \frac{\beta_{1}}{N}, \ldots, \frac{\beta_{d}}{N}\right)$ (où $N$ est un entier naturel $>0$, premier à l'idéal fractionnaire $\left(u_{0}\right)+\left(\beta_{1}\right)+\cdots+\left(\beta_{d}\right)$ de $\left.\mathcal{O}_{\mathbf{L}}\right)$ ne modifie pas le degré du corps de nombres $\mathbf{L}$ (ni, non plus, aucune des autres données) et transforme $\log |\Lambda|$ en $\log |\Lambda|-\log N$ ainsi que $h\left(1: u_{0}: \beta_{1}: \cdots: \beta_{d}\right)$ en $h(1:$ $\left.u_{0}: \beta_{1}: \cdots: \beta_{d}\right)+\log N\left(\operatorname{pour} N>\max \left\{\left|\overline{u_{0}}\right|, \ldots,\left|\overline{\beta_{d}}\right|\right\}\right)$. La contradiction arrive lorsque $N \rightarrow \infty$. Le majorant (雨) $\operatorname{de} \operatorname{deg} \pi(\widetilde{\mathbf{G}})$ est, quant à lui — et c'est nouveau — indépendant de $h(W)$.

Par ailleurs, avec des arguments ad hoc, il est possible de démontrer des majorations plus précises du degré de $\pi(\widetilde{\mathbf{G}})$ lorsque $\mathbf{G}$ est un groupe linéaire commutatif (voir $\S 3.3$ ci-après). Cependant, dans le cadre général d'un groupe algébrique commutatif quelconque, seule la méthode de Baker (que nous employons) permet, à l'heure actuelle, d'obtenir de tels renseignements. Par ailleurs, si nous nous sommes contentés de majorer le degré total de $\pi(\widetilde{\mathbf{G}})$, une adaptation triviale de la proposition 6.6 permet de majorer individuellement chaque coefficient de degré maximal du polynôme de Hilbert-Samuel multihomogène de $\pi(\widetilde{\mathbf{G}})$ (i.e. le degré des projections de $\pi(\widetilde{\mathbf{G}})$ sur ses ii $n$-faces d'indice $\left(i_{1}, \ldots, i_{n}\right)$ ¿¿i de $\left.\mathbf{G}_{1} \times \cdots \times \mathbf{G}_{n}\right)$, à l'instar du théorème 2 de [4].

D'autre part, il existe une constante $c_{6}$, ne dépendant que de $(\mathbf{G}, \Phi, \mathbf{e})$, telle que le terme $\max _{0 \leq s \leq \mathfrak{a}}\left\{h\left(s \mathbf{p}_{j}\right)\right\}$ soit majoré par

$$
c_{6} \mathfrak{a}^{\rho_{j}}\left(h\left(\mathbf{p}_{j}\right)+1\right) .
$$

Il était d'usage de remplacer le maximum par son majorant ${ }^{3}$. Mais cette majoration devient très maladroite lorsque le groupe $\mathbf{G}_{j}$ est un tore ou une variété abélienne. Dans le premier cas, par définition de la hauteur $h$, on a

$$
\max _{0 \leq s \leq \mathfrak{a}}\left\{h\left(s \mathbf{p}_{j}\right)\right\}=\max _{0 \leq s \leq \mathfrak{a}}\left\{h\left(\mathbf{p}_{j}^{s}\right)\right\}=\max _{0 \leq s \leq \mathfrak{a}}\left\{|s| h\left(\mathbf{p}_{j}\right)\right\}=\mathfrak{a} h\left(\mathbf{p}_{j}\right)
$$

et, dans le second cas, la hauteur $h$ est comparable à la hauteur de Néron-Tate $\widehat{h}$ (relative à une polarisation) de la variété abélienne $(i . e . h-\widehat{h}=O(1))$ et on a donc

$$
\max _{0 \leq s \leq \mathfrak{a}}\left\{h\left(s \mathbf{p}_{j}\right)\right\}=\mathfrak{a}^{2} \widehat{h}\left(\mathbf{p}_{j}\right)+O(1) .
$$

\footnotetext{
${ }^{3}$ Ce qui est source d'une erreur extrêmement fréquente dans la littérature 19, 20, 25], erreur déjà constatée par G. DiAz [15], qui consistait à majorer $h\left(\mathbf{p}_{j}\right)+1$ par une quantité $\log V_{j}$, définie comme un majorant de $\max \left\{\frac{1}{D}, h\left(\mathbf{p}_{j}\right), \frac{\left\|\mathbf{u}_{j}\right\|^{\rho_{j}}}{D}\right\}$ au lieu de $\max \left\{1, h\left(\mathbf{p}_{j}\right), \frac{\left\|\mathbf{u}_{j}\right\|^{\rho_{j}}}{D}\right\}$.
} 
Dans ces deux cas, nous évitons un terme $\mathfrak{a}^{\rho_{j}}$ supplémentaire et cette absence peut se révéler vraiment importante pour la qualité de la mesure lorsque (par exemple) $\mathbf{p}_{j}$ est un point de torsion du groupe $\mathbf{G}_{j}$.

En outre, en l'absence d'informations particulières sur le point $\mathbf{p}$ de $\mathbf{G}(\overline{\mathbb{Q}})$, il convient de remplacer, dans les inégalités (舟) et (5), le terme card $\left(\Gamma_{\mathbf{p}}(\mathfrak{a})\right)$ par $\mathfrak{a}+1$. Cependant, là encore, lorsque $\mathbf{p}$ est un point de torsion de $\mathbf{G}(\overline{\mathbb{Q}})$ (ou, encore mieux, si $\mathbf{p}=0$, i.e. le logarithme $\mathbf{u}$ appartient au sous-groupe des périodes de $\mathbf{G}(\mathbb{C})$ ), le cardinal de $\Gamma_{\mathbf{p}}(\mathfrak{a})$ est, a priori, très inférieur à $\mathfrak{a}+1$ et, concurremment avec la remarque précédente, les inégalités (4) et (5) deviennent beaucoup plus précises. Si $\mathbf{u} \in \Omega_{\mathbf{G}(\mathbb{C})}$, il n'est pas exclu qu'une modification de la construction de la fonction auxiliaire ou qu'un lemme de Schwarz approprié améliore fortement ces inégalités.

Quant au paramètre $E$, nous ne lui imposons aucune majoration, contrairement à l'usage établi (voir par exemple 12] et 19]). C'est en grande partie une question de présentation (voir remarque 2.9 de 19]), mais nous pensons que cela clarifie le lien entre les données et les paramètres. L'origine du paramètre $E$ est analytique. Il représente un quotient de rayons dans le lemme de Schwarz (approché).

Le calcul explicite des constantes qui apparaissent dans les théorèmes 3.1 et 3.2 nécessite la connaissance des données suivantes :

a) Pour chaque plongement $\sigma: \mathbf{K} \hookrightarrow \mathbb{C}$, les constantes $c_{\sigma}^{-}$et $c_{\sigma}^{+}$, définies par les inégalités (12) et (13), qui permettent de mesurer la ii taille analytique $i$ i du plongement $\Phi$ par rapport à la norme choisie sur $t_{\mathbf{G}_{\sigma}}(\mathbb{C})$.

b) Les constantes liées à l'écriture explicite des équations définissant le groupe $\mathbf{G}$ au voisinage de l'origine et aux formules d'additions et de dérivations dans le groupe $\mathbf{G}$ (p. 13).

c) La constante de la proposition 6.5. Cette constante peut être calculée pour un choix raisonnable des normes sur l'espace tangent et de $\Phi$ en reprenant les calculs de [5] et en s'appuyant en particulier sur la remarque 3 de cet article.

d) La hauteur d'un élément primitif $\alpha$ de $\mathbf{K}$ (i.e. tel que $\mathbf{K}=\mathbb{Q}(\alpha)$ ).

e) La constante qui apparaît dans la majoration du rang du système linéaire (lemme 6.18). Cependant, un plongement ii à la Serre ¿i, comme le nôtre, permet de prendre $8^{d}$ pour cette constante (voir le lemme 6.7 de 25]).

On notera que les constantes les plus difficiles à appréhender sont celles liées au plongement $\Phi$. Le mieux est donc d'éviter de plonger le groupe dans un espace projectif ! Le lecteur intéressé par cette question pourra consulter 18 (ainsi que la seconde partie de [17]) où l'utilisation de la méthode des pentes de J.-B. Bost permet de calculer cette constante dans le cas d'une variété abélienne.

Le terme $D h\left(\xi_{1}: \cdots: \xi_{D}\right)$, qui apparaît dans les inégalités (4), (5) et (5), résulte de la construction du polynôme auxiliaire avec une variante du lemme de Siegel. En réalité, ce terme est récurrent dans la littérature (voir, par exemple, le paragraphe 5 de [31] (condition (5.5)), le début de la démonstration de la proposition 3.8 de 30 (p. 275), et bien sûr [1, 12, 19, 20, 25, 26]) mais il ne représentait pas une contrainte réelle car, la plupart du temps sauf pour certaines mesures d'approximations simultanées, voir à ce sujet [20, 26]), il était inclus dans un terme général plus grand. Dans les cas favorables où la méthode des pentes (ou des déterminants d'interpolation) s'applique, cette quantité est supprimée. Lorsque le corps de nombres $\mathbf{L}$ est choisi ii minimal i¿ (comme corps de définition de $\mathbf{G}, W$ et $\mathbf{p}$ ), il existe une base $\xi_{1}, \ldots, \xi_{D}$ de $\mathbf{L}$ telle que la quantité $D h\left(\xi_{1}: \cdots: \xi_{D}\right)$ soit contrôlée. En effet, notons $\left(\beta_{0}^{(\ell)}: \cdots: \beta_{N_{\ell}}^{(\ell)}\right)$ l'image du point $\mathbf{p}_{\ell}$ par $\Phi$ dans $\mathbb{P}^{N_{\ell}}(\mathbf{L})(0 \leq \ell \leq n)$ et $\mathbf{K}_{\ell}$ le corps engendré sur $\mathbf{K}$ par les éléments $\beta_{j}^{(\ell)}, 0 \leq j \leq N_{\ell}$. Soit $\alpha$ un élément 
primitif de $\mathbf{K}_{\mid \mathbb{Q}}$. L'ensemble

$$
\left\{\alpha^{h} \beta_{1}^{i_{1}} \cdots \beta_{d}^{i_{d}} \prod_{\ell=0}^{n} \prod_{j=0}^{N_{\ell}}\left(\beta_{j}^{(\ell)}\right)^{m_{\ell, j}}\right\}
$$

où $i_{1}+\cdots+i_{d} \leq\left[\mathbf{K}\left(\beta_{1}, \ldots, \beta_{d}\right): \mathbf{K}\right], \sum_{j=0}^{N_{\ell}} m_{\ell, j} \leq\left[\mathbf{K}_{\ell}: \mathbf{K}\right]$ et $h \leq[\mathbf{K}: \mathbb{Q}]$, est un ensemble de générateurs du $\mathbb{Q}$-espace vectoriel $\mathbf{L}$. En particulier, $D$ étant le degré $[\mathbf{L}: \mathbb{Q}]$, on peut choisir une base $\xi_{1}=1, \xi_{2}, \ldots, \xi_{D}$ parmi ces générateurs. Cette base vérifie :

$$
\begin{aligned}
h\left(\xi_{1}: \cdots: \xi_{D}\right) \leq & {\left[\mathbf{K}\left(\beta_{1}, \ldots, \beta_{d}\right): \mathbf{K}\right] h\left(1: \beta_{1}: \cdots: \beta_{d}\right) } \\
& +\sum_{\ell=0}^{n}\left[\mathbf{K}_{\ell}: \mathbf{K}\right] h\left(\mathbf{p}_{\ell}\right)+[\mathbf{K}: \mathbb{Q}] h(\alpha) \\
\leq & D\left(h(W)+h\left(u_{0}\right)+h\left(\mathbf{p}_{1}\right)+\cdots+h\left(\mathbf{p}_{n}\right)\right) \\
& +[\mathbf{K}: \mathbb{Q}] h(\alpha) .
\end{aligned}
$$

Si cette majoration permet d'avoir une idée de la taille maximale de $D h\left(\xi_{1}: \cdots\right.$ : $\xi_{D}$ ), il serait maladroit de la substituer systématiquement à ce terme (par exemple si $\mathbf{L}=\mathbb{Q}$ ). L'utilisation du lemme de Bombieri-Vaaler [6] au lieu du lemme de Thue-Siegel doit permettre de remplacer la quantité $D h\left(\xi_{1}: \cdots: \xi_{D}\right)$ par le logarithme de la valeur absolue du discriminant (absolu) de L. Dans le cas particulier d'un produit de courbes elliptiques, S. DAVID \& N. HiRATA-Kohno ont montré qu'il était possible d'enlever ce terme si on utilisait pour la construction du polynôme auxiliaire un ii lemme de Siegel absolu i.i (voir 14]). Malheureusement, l'argument est fondé sur l'équivalence entre deux systèmes linéaires, et ce fait n'est plus nécessairement assuré avec un produit de groupes algébriques de dimension $>1$ lorsque nous sommes dans le ii cas périodique i.i. (voir définition 6.3).

Signalons également une légère modification du choix de $\mathfrak{a}$ dans le théorème 3.1 comparativement au théorème 0.1 de [16]. Cela ne modifie pas la démonstration mais la conséquence est que l'on n'a plus nécessairement $U$ supérieur à $D \log (D)$, terme qui apparaît conjointement avec $D h\left(\xi_{1}: \cdots \xi_{D}\right)$ lors de l'estimation de la hauteur du polynôme auxiliaire (voir inégalité (37), p. 27). C'est pourquoi nous l'avons rajouté dans les estimations (4), (5) et (6). De plus, on peut montrer que les théorèmes 3.1 et 3.2 restent valides en remplaçant $U$ (resp. a) par un majorant,

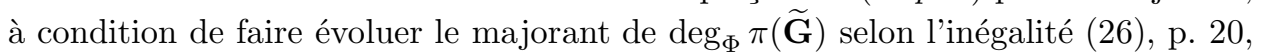
ce qui permet de retrouver ainsi le théorème 0.1 de op.cit.

Enfin, il est très probable que ce résultat correctement adapté (à l'instar de 14, 20]) reste valide lorsque $W$ est un sous-espace vectoriel de codimension $>1$ de $t_{\mathbf{G}}(\overline{\mathbb{Q}})$.

Afin de mieux percevoir la portée de la mesure (5) du théorème 3.1, nous allons mentionner plusieurs conséquences (simples) de ce théorème, lorsque le groupe $\mathbf{G}$ est réduit à un unique groupe algébrique $(n=1)$, en isolant chacune des quantités $h(\mathbf{p}), D,\|\mathbf{u}\|$. Les données sont celles du $\S$ 2, sans hypothèse particulière sur la nature du groupe algébrique $\mathbf{G}$.

Conséquence (Hauteur du point $\mathbf{p}$ ). Il existe une constante

$$
c_{7}=c_{7}\left(\mathbf{G}, \Phi, \mathbf{e}, \mathrm{d},\|\mathbf{u}\|, u_{0}, W, D\right),
$$

indépendante de $h(\mathbf{p})$, telle que si $\mathbf{u} \notin W$ alors

$$
\log \left|u_{0}-\beta_{1} u_{1}-\cdots-\beta_{d} u_{d}\right| \geq-c_{7} \frac{\max \{1, h(\mathbf{p})\}^{d}}{(\log \max \{e, h(\mathbf{p})\})^{d-1}} .
$$

Conséquence (Degré du corps de nombres L). Il existe une constante

$$
c_{8}=c_{8}\left(\mathbf{G}, \Phi, \mathbf{e}, \mathrm{d},\|\mathbf{u}\|, u_{0}, W, h(\mathbf{p})\right),
$$


indépendante de $D$, telle que si $\mathbf{u} \notin W$ alors

$$
\log \left|u_{0}-\beta_{1} u_{1}-\cdots-\beta_{d} u_{d}\right| \geq-c_{8} \frac{D^{2 d+2}}{(\log \max \{e, D\})^{d-1}} .
$$

Pour la conséquence suivante, nous prendrons $u_{0}=0$.

Conséquence (Norme du logarithme u). Il existe une constante

$$
c_{9}=c_{9}(\mathbf{G}, \Phi, \mathbf{e}, \mathrm{d}, W, D, h(\mathbf{p}))
$$

indépendante de $\|\mathbf{u}\|$, telle que si $\mathbf{u} \notin W$ alors

$$
\log \left|\beta_{1} u_{1}+\cdots+\beta_{d} u_{d}\right| \geq-c_{9} \max \{1,\|\mathbf{u}\|\}^{2 d}(\log \max \{e,\|\mathbf{u}\|\})^{d+2} .
$$

Les conséquences et améliorent, dans le cas général, les (meilleures) minorations (que nous avons déduites) de [19]:

et

$$
\log \left|u_{0}-\beta_{1} u_{1}-\cdots-\beta_{d} u_{d}\right| \geq-c_{10} \frac{\max \{1, h(\mathbf{p})\}^{d+1}}{(\log \max \{e, h(\mathbf{p})\})^{d}}
$$

$$
\log \left|\beta_{1} u_{1}+\cdots+\beta_{d} u_{d}\right| \geq-c_{11} \max \{1,\|\mathbf{u}\|\}^{2 d+2}(\log \max \{e, \| \mathbf{u} \mid\})^{d+1} .
$$

Ainsi, outre une mesure optimale en la hauteur de l'hyperplan, nous avons également amélioré la dépendance en la hauteur du point $\mathbf{p}$ et en la norme de son logarithme. La raison est que, au cours de la démonstration, nous avons remplacé la quantité $T \log T$ par $T \log \min \left\{D_{0}, T\right\}$ (T est un paramètre de dérivation et $D_{0}$ un degré partiel de polynôme). Comme le paramètre $T$ ne dépendait pas exclusivement de $h(W)$ (je pense, ici, à [12, 19, 25]), mais était aussi fonction de $h(\mathbf{p})$ et $\|\mathbf{u}\|$, la substitution du terme $T \log T$ au terme $T \log D_{0}$ apporte des modifications (en l'occurrence des améliorations) de la mesure. Ce n'est pas seulement $\log h(W)$ qui a disparu mais tout $\log \log B$ (avec les notations des articles cités auparavant). La dépendance en le degré du corps de nombres n'évolue pas. Le lecteur qui voudrait mieux comprendre ces points techniques est invité à se reporter au paragraphe 6.2 (ainsi qu'à l'annexe A de [17).

Avant de détailler le cas d'un groupe linéaire, montrons comment les théorèmes 1.1 et 1.2 ainsi que les conséquences, et se déduisent du théorème 3.1 .

Pour le théorème 1.1, choisissons $n=1$ et $\mathbf{G}_{1}$ la variété semi-abélienne en question. D'après les hypothèses, nous sommes dans le premier cas du théorème 3.1, et, compte tenu du fait que l'on peut remplacer $U$ par un majorant (voir remarque plus haut), on obtient

$$
\begin{aligned}
\operatorname{deg}_{\Phi} \pi(\widetilde{\mathbf{G}}) \leq \mathfrak{a} & \left(1+\frac{D \max _{s}\{h(s \mathbf{p})\}+(E\|\mathbf{u}\|)^{2}}{\log E}\right)^{\widetilde{d}} \\
& \times \max \left\{1, \frac{D h\left(\xi_{1}: \cdots: \xi_{D}\right)+D \log (D)}{U}\right\}
\end{aligned}
$$

où

et

$$
\mathfrak{a}=1+\left[\frac{D}{\log E} \log \left(1+\frac{D+h(\mathbf{p})+(E\|\mathbf{u}\|)^{2}}{\log E}\right)\right]
$$

$$
U=\mathfrak{a}(D h(W)+\mathfrak{a} \log E)\left(1+\frac{D \max _{s}\{h(s \mathbf{p})\}+(E\|\mathbf{u}\|)^{2}}{\log E}\right)^{d} .
$$

En utilisant la majoration $(7)$, on a $h\left(\xi_{1}: \cdots: \xi_{D}\right)+\log (D) \leq U$ puis l'estimation générale $\max _{s \leq \mathfrak{a}}\{h(s \mathbf{p})\} \leq c_{12} \mathfrak{a}^{2}(h(\mathbf{p})+1)$, on obtient le théorème 1.1. Quant au théorème 1.2, il vient de ce que $U$ et $h\left(\xi_{1}: \cdots: \xi_{D}\right)$ peuvent être majorés par une fonction linéaire de la hauteur de $W$. La conséquence provient de l'inégalité (5) en choisissant $E=\sqrt{\max \{e, h(\mathbf{p})\}}$. De même la conséquence (resp. ) vient en choisissant $E=\sqrt{D}$ (resp. $E=c_{13}$ où $c_{13}$ est une constante qui ne dépend pas de $\|\mathbf{u}\|)$. 
3.3. Cas d'un groupe linéaire commutatif. Dans ce paragraphe, nous détaillons le cas (classique) où $\mathbf{G}$ est le produit du groupe additif $\mathbb{G}_{\mathrm{a}}$ par un tore.

Corollaire 3.3. Pour tout entier $k \geq 1$, il existe une constante $c(k)>0$ ayant la propriété suivante. Soient $u_{1}, \ldots, u_{k}$ des nombres complexes tels que chaque $\alpha_{\ell}:=e^{u_{\ell}}$ appartienne $\grave{a} \overline{\mathbb{Q}}$. Soient $u_{0}, \beta_{1}, \ldots, \beta_{k}$ des nombres algébriques non tous nuls. Soient $D$ le degré du corps de nombres $\mathbf{M}:=\mathbb{Q}\left(\alpha_{1}, \ldots, \alpha_{k}, u_{0}, \beta_{1}, \ldots, \beta_{k}\right)$ et $\xi_{1}, \ldots, \xi_{D}$ une base du $\mathbb{Q}$-espace vectoriel $\mathbf{M}$. Soit $E$ un réel $\geq$ e. Posons $\Lambda:=$ $u_{0}+\beta_{1} u_{1}+\cdots+\beta_{k} u_{k}$,

$$
\mathfrak{a}^{\prime}:=1+\left[\frac{D}{\log E} \log \left(1+\frac{\left.D+h\left(\alpha_{1}\right)+\cdots+h\left(\alpha_{k}\right)+E\left|u_{1}\right|+\cdots+E\left|u_{k}\right|\right)}{\log E}\right)\right],
$$

et

$$
\begin{aligned}
V:= & \operatorname{card}\left(\Gamma_{\mathbf{p}}\left(\mathfrak{a}^{\prime}\right)\right) \times\left(D h\left(1: u_{0}: \beta_{1}: \cdots: \beta_{k}\right)+\mathfrak{a}^{\prime} \log E\right) \\
& \times \prod_{j=1}^{k}\left(1+\frac{D h\left(\alpha_{j}\right)+E\left|u_{j}\right|}{\log E}\right) .
\end{aligned}
$$

Premier cas : $\Lambda=0$.

Alors $u_{0}=0$ et il existe une $\mathbb{Z}$-base $e_{1}, \ldots, e_{k}$ de $\mathbb{Z}^{k}$, un entier naturel $0<q<k$ et des entiers $1 \leq j_{1}<\cdots<j_{k-q} \leq k$, tels que

a) Le $k$-uplet $\left(\beta_{1}, \ldots, \beta_{k}\right)$ appartient au $\mathbf{M}$-espace vectoriel engendré par $e_{1}, \ldots, e_{q}$.

b) $S i e_{\ell, 1}, \ldots, e_{\ell, k}$ désignent les coordonnées de $e_{\ell}$ dans la base canonique de $\mathbb{Z}^{k}$, alors, pour tout $\ell \in\{1, \ldots, q\}$, on a $e_{\ell, 1} u_{1}+\cdots+e_{\ell, k} u_{k}=0$.

c) Si $\mathcal{M}$ désigne la matrice $\left(e_{\ell, i}\right)_{\substack{1 \leq \ell \leq q \\ 1 \leq i \leq k}} \in \operatorname{Mat}_{q, k}(\mathbb{Z})$ alors

$$
\begin{aligned}
\left(\operatorname{det} \mathcal{M}^{\mathrm{t}} \mathcal{M}\right)^{1 / 2} \leq & c(k) \operatorname{card}\left(\Gamma_{\mathbf{p}}\left(\mathfrak{a}^{\prime}\right)\right)\left\{\prod_{\ell=1}^{k-q}\left(1+\frac{D h\left(\alpha_{j_{\ell}}\right)+E\left|u_{j_{\ell}}\right|}{\log E}\right)\right\} \\
& \times \max \left\{1, \frac{D h\left(\xi_{1}: \cdots: \xi_{D}\right)+D \log (D)}{V}\right\}
\end{aligned}
$$

où ${ }^{\mathrm{t}} \mathcal{M}$ est la matrice transposée de $\mathcal{M}$.

Second cas : $\Lambda \neq 0$.

Alors on a

$$
\log |\Lambda| \geq-c(k) \max \left\{V, D h\left(\xi_{1}: \cdots: \xi_{D}\right)+D \log (D)\right\} .
$$

De plus, si $u_{0} \neq 0$ et

$$
\begin{aligned}
V^{\prime}:= & \mathfrak{a}^{\prime}\left(D h\left(1: \beta_{1}: \cdots: \beta_{k}\right)+\max _{0 \leq s \leq c(k) \mathfrak{a}^{\prime}}\left\{D h\left(s u_{0}\right)\right\}+\log E\right) \\
& \times \prod_{j=1}^{k}\left(1+\frac{D h\left(\alpha_{j}\right)+E\left|u_{j}\right|}{\log E}\right),
\end{aligned}
$$

alors

$$
\log |\Lambda| \geq-c(k) \max \left\{V^{\prime}, D h\left(\xi_{1}: \cdots: \xi_{D}\right)+D \log (D)\right\} .
$$

Démonstration. La démonstration de ce corollaire à partir des théorèmes 3.1 et 3.2 repose essentiellement sur la structure des sous-groupes algébriques d'un groupe linéaire commutatif (voir, par exemple, la proposition 5.6 de [33], p. 157).

- Si $\Lambda=0$, d'après le théorème 3.1 appliqué à $\mathbf{G}=\mathbb{G}_{\mathrm{a}} \times \mathbb{G}_{\mathrm{m}}^{k}$, il existe un sous-groupe algébrique connexe $\widetilde{\mathbf{G}}$ de $\mathbf{G}$ vérifiant les points suivants :

a) Il existe un sous-groupe $\widetilde{Z}$ de $\mathbb{Z}^{k}$ tel que $\widetilde{\mathbf{G}}=\{0\} \times \mathbf{T}_{\widetilde{Z}}$, où, si $A$ est un anneau (commutatif), $\mathbf{T}_{\widetilde{Z}}(A)=\left\{\underline{x} \in A^{k} ; \underline{x} \underline{\underline{m}}=1\right.$ pour tout $\left.\underline{m} \in \widetilde{Z}\right\}$. Notons $q$ le rang de $\widetilde{Z}$. Soient $e_{1}, \ldots, e_{k}$ une base (adaptée à $\widetilde{Z}$ ) de $\mathbb{Z}^{k}$ et $a_{1}, \ldots, a_{q}$ des entiers naturels non nuls tels que $\widetilde{Z}:=\mathbb{Z} . a_{1} e_{1} \oplus \cdots \oplus \mathbb{Z} . a_{q} e_{q}$. 
Comme $\widetilde{\mathbf{G}}$ est connexe, le $\mathbb{Z}$-module $\widetilde{Z}$ est facteur direct dans $\mathbb{Z}^{k}(\widetilde{Z}$ est un sous-groupe primitif de $\left.\mathbb{Z}^{k}\right)$, i.e. $\mathbb{Z}^{k} / \widetilde{Z}$ est sans torsion. Par conséquent, les entiers $a_{i}$ sont égaux à 1 . Notons que la partie additive de $\widetilde{\mathbf{G}}$ est nulle car sinon on aurait $t_{\mathbb{G}_{\mathrm{a}}} \subseteq\left\{z_{0}=\beta_{1} z_{1}+\cdots+\beta_{k} z_{k}\right\}$. De plus, l'espace tangent $t_{\widetilde{\mathbf{G}}}=t_{\mathbf{T}_{\tilde{Z}}}$ s'identifie à l'ensemble

$\widetilde{Z}^{\perp}=\left\{\left(x_{1}, \ldots, x_{k}\right) \in \mathbf{M}^{k} ; x_{1} m_{1}+\cdots+x_{k} m_{k}=0, \forall\left(m_{1}, \ldots, m_{k}\right) \in \widetilde{Z}\right\}$

(voir Lemma 8.13 de [33]). L'inclusion de $t_{\mathbf{T}_{\tilde{Z}}}$ dans l'hyperplan $W:=$ $\left\{\beta_{1} z_{1}+\cdots+\beta_{k} z_{k}=0\right\}$ se traduit alors par

$$
W^{\perp}=\mathbf{M} \cdot\left(\beta_{1}, \ldots, \beta_{k}\right) \subseteq \widetilde{Z} \otimes \mathbf{M} .
$$

b) L'appartenance de $\left(u_{0}, u_{1}, \ldots, u_{k}\right)$ à $t_{\widetilde{\mathbf{G}}}(\mathbb{C})$ signifie précisément que $u_{0}=0$ et $e_{\ell, 1} u_{1}+\cdots+e_{\ell, k} u_{k}=0$, pour tout $\ell \in\{1, \ldots, q\}$.

c) Le degré du groupe $\pi(\widetilde{\mathbf{G}})=\mathbf{T}_{\widetilde{Z}}$, relatif au plongement usuel de $\mathbf{G}$ dans $\left(\mathbb{P}^{1}\right)^{k+1}$, est, à une constante près ne dépendant que de $k$, égal à la somme des valeurs absolues des mineurs maximaux de la matrice $\mathcal{M}$ et est donc équivalent à $\sqrt{\operatorname{det}\left(\mathcal{M}^{\mathrm{t}} \mathcal{M}\right)}$, en vertu de la formule de Cauchy-Binet (pour plus de détails, on peut se reporter à la démonstration de la proposition 4 de (5). L'inégalité (8) résulte alors de la majoration (4) de $\operatorname{deg} \pi(\widetilde{\mathbf{G}})$.

- Si $\Lambda \neq 0$, les minorations de $\log |\Lambda|$ sont les conséquences directes des inégalités (5) et $(6)$.

Ce résultat entraîne le théorème célèbre de BAKER :

Théorème . Si $\alpha_{1}, \ldots, \alpha_{k}$ sont des nombres algébriques dont les logarithmes sont linéairement indépendants sur $\mathbb{Q}$ alors $1, \log \alpha_{1}, \ldots$,

$\log \alpha_{k}$ sont linéairement indépendants sur $\overline{\mathbb{Q}}$.

La majoration du déterminant de la première partie du corollaire 3.3 s'inscrit dans la lignée des travaux sur les relations de dépendance linéaire entre les logarithmes de nombres algébriques. Elle est une conséquence de la démonstration de la mesure d'indépendance linéaire. Pour obtenir des ii petites i.i relations de dépendance linéaire, il est parfois plus efficace d'attaquer le problème directement (donc sans fonction auxiliaire, extrapolation, ...) à l'aide, par exemple, de la géométrie des nombres (voir lemme 7.19. de [33], p. 222). Ce problème est lié aux relations de dépendance multiplicative entre nombres algébriques, étudié par D. MAsser dans [22], et à l'étude du covolume du sous-groupe de $\mathbb{Z}^{k}$ :

$$
\left\{\left(b_{1}, \ldots, b_{k}\right) \in \mathbb{Z}^{k} ; \alpha_{1}^{b_{1}} \cdots \alpha_{k}^{b_{k}}=1\right\} .
$$

Un majorant du covolume est de la forme

$$
c(k, \mathbf{L}) \times \max _{1 \leq j_{1}<\cdots<j_{k-q} \leq k}\left\{\prod_{\ell=1}^{k-q} h\left(\alpha_{j_{\ell}}\right)\right\} .
$$

Un théorème plus précis se trouve p. 205 de [4] (voir également [3]).

Par ailleurs, le corollaire 3.3 ne contient pas les résultats quantitatifs déjà connus pour les sous-groupes linéaires commutatifs (comme, par exemple, le théorème 9.1 de [33]), ne serait-ce que parce que, contrairement à nous, les auteurs précisent la constante $c(k)$. Dans le cas non-homogène $\left(u_{0} \neq 0\right)$, le terme

$$
\mathfrak{a}^{\prime} \times\left(D h\left(1: \beta_{1}: \cdots: \beta_{k}\right)+\max _{s}\left\{D h\left(s u_{0}\right)\right\}+\log E\right)
$$

devrait plutôt être remplacé par

$$
\operatorname{Dh}\left(1: u_{0}: \beta_{1}: \cdots: \beta_{k}\right)+\mathfrak{a}^{\prime}+\log E
$$

(theorem 9.1 de [33], p. 251). L'explication de cette anomalie provient d'une utilisation (encore) trop ii grossière i. du lemme de zéros de P. PHILIPPon, car, contrairement à [33], nous n'avons pas supposé que les logarithmes $u_{1}, \ldots, u_{k}$ étaient 
linéairement indépendants ${ }^{4}$ sur $\mathbb{Q}$. A fortiori, avec la mesure d'indépendance (9), nous ne retrouvons pas les mesures fines relatives au ii cas rationnel homogène $i, \dot{i}$, qui concerne les minorations de $\left|b_{1} \log \alpha_{1}+\cdots+b_{k} \log \alpha_{k}\right|$ avec $b_{i} \in \mathbb{Q}$ et $\alpha_{j} \in \overline{\mathbb{Q}}$ (le groupe algébrique sous-jacent étant $\mathbb{G}_{\mathrm{m}}^{k}$ ou $\mathbb{G}_{\mathrm{a}}^{k-1} \times \mathbb{G}_{\mathrm{m}}$ selon que l'on utilise la méthode de Baker ou de Schneider, voir [33, chapitre 14.4), ces mesures étant obtenues à partir de méthodes et d'outils spécifiques comme les polynômes de Fel'dman.

\section{Organisation Du TEXTE}

La démonstration des théorèmes 3.1 et 3.2 fait intervenir l'existence de plongements des groupes $\mathbf{G}_{i}$ dans des espaces projectifs, plongements qui possèdent des propriétés supplémentaires ii à la Serre i.i. Ces outils sont détaillés dans le paragraphe suivant. Ensuite vient la preuve des théorèmes 3.1 et 3.2. Tout d'abord (§ 6.1), nous ii justifions i.i le choix des données de départ (en particulier l'hyperplan $W$, transversal aux espaces tangents $t_{\mathbf{G}_{0}}$ et $\left.t_{\mathbf{G}_{1}} \oplus \cdots \oplus t_{\mathbf{G}_{n}}\right)$. Nous choisissons alors des paramètres de manière à ce que la section ${ }^{5}$ d'un fibré ample sur $\mathbf{G}$, qui sera construite avec ces paramètres, ne puisse s'annuler le long de l'hyperplan avec un ordre de multiplicité élevé en les premiers multiples d'un point $\mathbf{p}\left(:=\exp _{\mathbf{G}(\mathbb{C})}(\mathbf{u})\right)$ sans être identiquement nulle $(\S 6.2)$. Le choix de ces paramètres est guidé par le souci de prendre en compte d'emblée le ii sous-groupe obstructeur ¿i. fourni par le lemme de zéros de P. PhiLipPon 23. La contrainte liée à ce type de méthode est que, dans le reste de la démonstration, nous devons garder (jusqu'à l'extrapolation analytique) un sous-groupe obstructeur particulier qui conditionne le type d'extrapolation effectué au $\S 6.10$, selon qu'un multiple de $\mathbf{p}$ appartienne ou non à ce sous-groupe (distinction entre cas périodique et cas non-périodique, selon la terminologie introduite par P. Philippon \& M. WALDschmidt [25], fin du paragraphe 6.2]). Dans le cas périodique, nous donnons quelques informations complémentaires sur le sousgroupe obstructeur, qui seront à l'origine de la majoration (41) du théorème 3.1 (§ 6.4). Après quelques énoncés techniques concernant le choix de bases pour l'hyperplan ( $\$ 6.3$ et 6.6) et les modifications des dérivées liées au passage d'une base à une autre ( $\$ 6.7)$, nous entrons dans le vif de la démonstration avec la construction de la fonction auxiliaire $(\S 6.8$ et 6.9 ) puis nous extrapolons ii à la Baker i.j. (dans le cas non-périodique seulement, le cas périodique subissant un traitement différent). Un paramétrage local directement lié au groupe algébrique et non plus à son espace tangent nous permet d'apporter une amélioration essentielle dans l'estimation arithmétique de la première dérivée non-nulle de la fonction auxiliaire. Le résultat du paragraphe 6.11 est au centre de la démonstration des théorèmes 3.1 et 3.2, qui découle alors d'un raisonnement par l'absurde.

\section{Plongements des groupes algÉbriques}

Pour effectuer les calculs analytiques, nous aurons besoin d'une représentation de l'application exponentielle avec des fonctions holomorphes et d'un plongement de $\mathbf{G}$ dans un espace projectif. Nous présentons ici les propriétés que nous pouvons attendre d'un tel plongement, en particulier vis-à-vis de la représentation de la loi d'addition du groupe. Ce procédé a l'inconvénient d'introduire des constantes liées à ce plongement, théoriquement effectives, mais difficile à calculer explicitement (voir $\S 3.2$ ).

Résultats généraux. Soit $\mathbf{H}$ un groupe algébrique commutatif, défini sur un

\footnotetext{
${ }^{4}$ Cette hypothèse supplémentaire permet, par exemple, de calculer le cardinal des quotients $\left(\Gamma_{\mathbf{p}}(S)+\mathbf{G}^{\prime}(\mathbf{L})\right) / \mathbf{G}^{\prime}(\mathbf{L})$ qui apparaissent si on utilise le lemme de zéros [23.

${ }^{5} \mathrm{Ce}$ vocabulaire géométrique n'est pas repris au cours de la démonstration.
} 
corps de nombres $\mathbf{K}$, de dimension $d$. Soit $\iota: \mathbf{H} \hookrightarrow \mathbb{P}_{\mathbf{K}}^{N}$ un plongement (quasiprojectif). Si $X_{0}, \ldots, X_{N}$ désignent les coordonnées sur l'espace projectif $\mathbb{P}_{\mathbf{K}}^{N}$, on note $s_{\ell}=\iota^{*}\left(X_{\ell}\right)$. Ainsi, pour $x \in \mathbf{G}$, on a $\iota(x)=\left(s_{0}(x): \cdots: s_{N}(x)\right)$. D'après [28], il est possible de choisir $\iota$ de telle façon que chacun des groupes de Lie complexes $\mathbf{H}_{\sigma}(\mathbb{C})$, pour $\sigma: \mathbf{K} \hookrightarrow \mathbb{C}$ plongement archimédien de $\mathbb{K}$ dans $\mathbb{C}$, admettent une représentation de leur exponentielle $\exp _{\sigma}$ par les fonctions holomorphes ${ }^{6}$ $\varphi_{\ell}: t_{\mathbf{H}_{\sigma}}(\mathbb{C}) \rightarrow s_{\ell}\left(\exp _{\sigma}(z)\right)$, avec les propriétés suivantes $(c f$. [32, en particulier les propriétés 4.4 et 4.6, p. 76$)$ :

Normalisations.

- Les fonctions $\varphi_{j}$ sont des fonctions analytiques d'ordre $\rho$ inférieur ou égal à 2 et sans zéro commun dans $t_{\mathbf{H}_{\sigma}}(\mathbb{C})$.

- $\left(\varphi_{0}(0), \cdots, \varphi_{N}(0)\right)=(1,0, \ldots, 0)$

- Soient $\mathbf{e}_{\mathbf{H}_{\sigma}}$ une base de $t_{\mathbf{H}_{\sigma}}(\mathbb{C})$ et $z_{1}, \ldots, z_{d}$ les coordonnées locales liées à cette base. À l'origine, la matrice jacobienne

$$
\left(\frac{\partial\left(\varphi_{\ell} / \varphi_{0}\right)}{\partial z_{i}}(0)\right)_{\substack{1 \leq \ell \leq d \\ 1 \leq i \leq d}}
$$

est la matrice identité (cette propriété n'est rien d'autre que la lissité du groupe G à l'origine).

Croissance analytique.

- Soit $\|\cdot\|_{\sigma}$ une norme hermitienne sur $t_{\mathbf{H}_{\sigma}}(\mathbb{C})$. Il existe deux constantes positives $c_{\sigma}^{-}$et $c_{\sigma}^{+}$telles que, pour tout $z \in t_{\mathbf{H}_{\sigma}}(\mathbb{C})$, on ait

$$
\exp \left\{-c_{\sigma}^{-}\left(1+\|z\|_{\sigma}\right)^{\rho}\right\} \leq \max \left\{\left|\varphi_{0}(z)\right|, \ldots,\left|\varphi_{N}(z)\right|\right\}
$$

et

$$
\max \left\{\left|\varphi_{0}(z)\right|, \ldots,\left|\varphi_{N}(z)\right|\right\} \leq \exp \left\{c_{\sigma}^{+}\left(1+\|z\|_{\sigma}\right)^{\rho}\right\}
$$

Loi d'addition.

- Il existe une constante $c_{\mathbf{H}_{\sigma}}^{(1)}$ vérifiant : pour tout $x \in t_{\mathbf{H}_{\sigma}}(\mathbb{C})$, il existe un voisinage $\mathcal{V}_{x}$ de 0 dans $t_{\mathbf{H}_{\sigma}}(\mathbb{C})$ et $N+1$ polynômes $A_{0}, \ldots, A_{N}$ de $\mathbf{K}[\mathbf{X}, \mathbf{Y}]$, où $\mathbf{X}=\left(X_{0}, \ldots, X_{N}\right)$ et $\mathbf{Y}=\left(Y_{0}, \ldots, Y_{N}\right)$, bi-homogènes, tous de même bidegré, de degré total $\leq c_{\mathbf{H}_{\sigma}}^{(1)}$, tels que, pour tout $z \in \mathcal{V}_{x}$, les éléments

$$
\left(\varphi_{0}(z+x): \cdots: \varphi_{N}(z+x)\right)
$$

et

$$
\left(A_{0}(\varphi(z), \varphi(x)): \cdots: A_{N}(\varphi(z), \varphi(x))\right)
$$

soient égaux.

Structure différentielle.

- Il existe une constante $c_{\mathbf{H}_{\sigma}}^{(2)}$ vérifiant : pour tout triplet $(k, j, h)$ avec $k \in$ $\{1, \ldots, d\}$ et $j, h \in\{0, \ldots, N\}$, il existe un polynôme $C_{k j h} \in \mathbf{K}[\mathbf{X}]$, de degré total $\leq c_{\mathbf{H}_{\sigma}}^{(2)}$, tel que

$$
\frac{\partial}{\partial z_{k}}\left(\frac{\varphi_{j}}{\varphi_{h}}\right)=C_{k j h}\left(\frac{\varphi_{0}}{\varphi_{h}}, \ldots, \frac{\varphi_{N}}{\varphi_{h}}\right) .
$$

(où les coordonnées $z_{i}$ sont celles considérées plus haut (relativement à la base $\left.\mathbf{e}_{\mathbf{H}_{\sigma}}\right)$ ).

\footnotetext{
${ }^{6}$ Pour ne pas alourdir excessivement les notations, nous omettrons la référence au plongement $\sigma$; dans le contexte, il ne devrait pas y avoir de confusion possible.
} 
Notations pour la démonstration ( $\S \mathbf{6}$ ). Lorsque $\mathbf{H}$ est le groupe produit $\mathbf{G}_{0} \times \cdots \times \mathbf{G}_{n}$, les résultats précédents s'appliquent à chacun des groupes $\mathbf{G}_{i}$. L'exponentielle complexe de $\mathbf{G}_{i, \sigma_{0}}(\mathbb{C})$ sera représentée par $\varphi^{(i)}=\left(\varphi_{0}^{(i)}, \ldots, \varphi_{N_{i}}^{(i)}\right)$ pour $i \in\{0, \ldots, n\}$ (chacun des groupes $\mathbf{G}_{i}$ se plonge dans un espace projectif $\mathbb{P}_{\mathbf{K}}^{N_{i}}$ ). Chacune des fonctions (holomorphes sur $\left.t_{\mathbf{G}_{i}}(\mathbb{C})\right) \varphi_{h}^{(i)}$ est d'ordre $\leq \rho_{i}$. Nous noterons également $A_{h}^{(i)}$ (resp. $C_{k j h}^{(i)}$ ) les polynômes provenant des lois d'additions de $\mathbf{G}_{i}$ (resp. des propriétés différentielles de $\mathbf{G}_{i}$, relativement à la base $\mathbf{e}_{i}$ définie au $\S 2.1)$. Ce sont des polynômes en les variables $\mathbf{X}_{i}:=\left(X_{0}^{(i)}, \ldots, X_{N_{i}}^{(i)}\right)$ et $\mathbf{Y}_{i}:=$ $\left(Y_{0}^{(i)}, \ldots, Y_{N_{i}}^{(i)}\right)$. Enfin, nous désignerons par $\psi_{h}^{(i)}: t_{\mathbf{G}_{i, \sigma}}(\mathbb{C}) \rightarrow \mathbb{C}^{N_{i}+1}$ l'application méromorphe

$$
\left(\frac{\varphi_{0}^{(i)}}{\varphi_{h}^{(i)}}, \ldots, \frac{\varphi_{h-1}^{(i)}}{\varphi_{h}^{(i)}}, 1, \frac{\varphi_{h+1}^{(i)}}{\varphi_{h}^{(i)}}, \ldots, \frac{\varphi_{N_{i}}^{(i)}}{\varphi_{h}^{(i)}}\right) .
$$

Nous noterons $\|\cdot\|_{j, \sigma}$ (ou $\|\cdot\|_{j}$ si $\sigma$ est le plongement $\sigma_{0}$ de $\mathbf{K}$ dans $\mathbb{C}$ choisi au $\S 2.1)$ la norme hermitienne sur $t_{\mathbf{G}_{j, \sigma}}(\mathbb{C})$ obtenue par transfert de la norme hermitienne canonique sur $\mathbb{C}^{\delta_{j}}$ via l'isomorphisme $t_{\mathbf{G}_{j, \sigma}}(\mathbb{C}) \simeq \mathbb{C}^{\delta_{j}}$ fixé par la base $\sigma\left(\mathbf{e}_{j}\right)$ de $t_{\mathbf{G}_{j}} \otimes_{\sigma(\mathbf{K})} \mathbb{C}$. La norme $\|\cdot\|_{\sigma}$ sera la norme induite par somme directe orthonormale sur $t_{\mathbf{G}_{\sigma}}(\mathbb{C})$. Enfin, $\Phi$ désignera l'application analytique de $t_{\mathbf{G}}(\mathbb{C})$ dans $\mathbb{C}^{\left(N_{0}+1\right)+\cdots+\left(N_{n}+1\right)}$ qui à $z$ associe

$$
\left(\left(\varphi_{0}^{(0)}(z), \ldots, \varphi_{N_{0}}^{(0)}(z)\right), \ldots,\left(\varphi_{0}^{(n)}(z), \ldots, \varphi_{N_{n}}^{(n)}(z)\right)\right) .
$$

Cette application représente l'exponentielle de $\mathbf{G}_{\sigma_{0}}(\mathbb{C})$.

Remarque 5.1. Ici $\mathbf{G}_{0}:=\mathbb{G}_{\mathrm{a}}$ est plongé dans $\mathbb{P}^{1}\left(N_{0}=1\right)$ par l'application usuelle $z_{0} \mapsto\left(1: z_{0}\right)$.

\section{DÉmonstration Des théorèmes 3.1 ET 3.2}

6.1. Réductions. N. Hirata-Kohno a remarqué [19 que l'ajout d'un groupe affine $\mathbb{G}_{\mathrm{a}}$ au groupe donné naturellement par le problème (ici $\mathbf{H}:=\mathbf{G}_{1} \times \cdots \mathbf{G}_{n}$ ) a un rôle modérateur sur les dérivations. L'adjonction ii artificielle i¿ de ce facteur $\mathbb{G}_{\mathrm{a}}$ à $\mathbf{G}$ revient à minorer $\left|1.0-\left(\beta_{1} u_{1}+\cdots+\beta_{d} u_{d}\right)\right|$. La présence de ce facteur $\mathbb{G}_{\mathrm{a}}$ contrebalance l'influence négative des dérivations sur les coefficients $\beta_{i}$ de la manière suivante. Dériver, à l'ordre $T$, le long de l'hyperplan $z_{0}=\beta_{1} z_{1}+\cdots+\beta_{d} z_{d}$, la fonction $P \circ \exp _{\left(\mathbb{G}_{\mathrm{a}} \times \mathbf{H}\right)}$ (où $P$ est un polynôme multihomogène), au point 0 , équivaut à dériver $F_{W}(\underline{z}):=P\left(\beta_{1} z_{1}+\cdots+\beta_{d} z_{d}, \exp _{\mathbf{H}}\left(z_{1} e_{1}+\cdots+z_{d} e_{d}\right)\right)$ (la notation ici se veut plus suggestive que rigoureuse) dans toutes les directions $\frac{\partial}{\partial z_{i}}(i=1, \ldots, d)$, et cela fait apparaître, dans les coefficients de Taylor de $F_{W}$ d'ordre $T$, une dépendance polynomiale en les $\beta_{i}$ de degré majoré par $\min \left\{\operatorname{deg}_{X_{0}} P, T\right\}$ (au lieu de $T$ seulement). Dans ce texte, cette astuce a été intégrée dans le choix des données de départ (voir équation (2)). Ce n'est pas une restriction car il suffit de modifier $u_{0}$ (par exemple, $u_{0}=0$ pour une forme homogène) pour recouvrir tous les cas possibles. Cette transformation de la forme linéaire et du logarithme considérés, qu'il est de coutume de désigner sous l'appellation ii réduction de N. Hirata-Kohno ii. (il en existe plusieurs variantes), s'avère cruciale dans la suite. Le lecteur intéressé par plus de détails pourra consulter le chapitre 4 de 12 .

C'est pourquoi, sans perte de généralités, nous supposerons que les deux conditions suivantes sont satisfaites.

i) Aucun des espaces tangents $t_{\mathbf{G}_{i}}$ n'est inclus dans $W$ (i.e. que la famille des $\beta_{j}$ correspondant aux variables de $t_{\mathbf{G}_{i}}$ n'est pas réduite à $\left.\{0\}\right)$.

ii) $\operatorname{Max}\left\{\left|u_{0}\right|,\left|\beta_{m}\right|_{1 \leq m \leq d}\right\}=1$. En effet, si on note $\mathfrak{x}$ un élément de $\left\{u_{0}\right\} \cup$ $\left\{\beta_{m}\right\}_{1 \leq m \leq d}$, de valeur absolue maximale, alors

$$
D h\left(u_{0}\right)+D h(W) \geq \log |\mathfrak{x}| \geq-D h\left(u_{0}\right)-D h(W)
$$


et il est donc équivalent de minorer $\log |\Lambda|$ et

$$
\log \left|\frac{u_{0}}{\mathfrak{x}}-\sum_{m=1}^{d}\left(\frac{\beta_{m}}{\mathfrak{x}}\right) \cdot u_{m}\right| .
$$

La première hypothèse permet d'assurer que les entiers $D_{i}, 1 \leq i \leq n$, construits au paragraphe suivant, ne sont pas nuls (voir détails p. 23). La seconde hypothèse est utile (uniquement) dans le cas périodique pour l'extrapolation analytique.

6.2. Choix des paramètres. Soient $C_{0}$ un réel positif et $E \geq e$. Au cours de la démonstration, nous dirons souvent qu'une inégalité est vraie ii pour $C_{0}$ assez grand i¿. Cela signifie qu'il existe une constante ne dépendant que de $(\mathbf{G}, \Phi, \mathbf{e}, \mathrm{d})$ telle que si $C_{0}$ est supérieur à cette constante alors l'inégalité est vraie. Dans le contexte, la constante en question sera facile à calculer (voir commentaires du $\S 3.1$ ) et, comme la preuve ne fait apparaître qu'un nombre fini de telles constantes, le réel $C_{0}$ peut être choisi comme le maximum de ces constantes.

Définissons

$$
S:=\left[C_{0}^{5} \mathfrak{a}\right], \quad S_{0}:=\left[C_{0}^{3} \mathfrak{a}\right] .
$$

On pourra noter que $D \log S \leq S \log E$ pour $C_{0}$ assez grand. Pour définir les paramètres suivants, nous aurons à distinguer le cas général du cas particulier où $\prod_{j=1}^{n} \mathbf{G}_{j}$ est une variété semi-abélienne et $u_{0} \neq 0$. Rappelons que $\mathbf{p}:=\exp _{\mathbf{G}_{\sigma_{0}}(\mathbb{C})}(\mathbf{u})$ et posons :

$$
\begin{aligned}
U_{0}:=\max \{ & C_{0}^{4 d-1} \frac{\operatorname{card}\left(\Gamma_{\mathbf{p}}(S)\right)}{(S \log E)^{d}}\left(D h(W)+D h\left(u_{0}\right)+S \log E\right) \\
& \times \prod_{j=1}^{n}\left(S \log E+\max _{0 \leq s \leq(d+1) S}\left\{D h\left(s \mathbf{p}_{j}\right)\right\}+\left(E S\left\|\mathbf{u}_{j}\right\|\right)^{\rho_{j}}\right)^{\delta_{j}}, \\
& \left.C_{0} D\left(h\left(\xi_{1}: \cdots: \xi_{D}\right)+\log (D)\right)\right\}
\end{aligned}
$$

resp.

$$
\begin{aligned}
U_{0}^{\prime}:=\max \{ & C_{0}^{4 d-1} \frac{S}{(S \log E)^{d}}\left(D h(W)+\max _{0 \leq s \leq(d+1) S}\left\{D h\left(s u_{0}\right)\right\}+C_{0}^{2} \log E\right) \\
& \times \prod_{j=1}^{n}\left(S \log E+\max _{0 \leq s \leq(d+1) S}\left\{D h\left(s \mathbf{p}_{j}\right)\right\}+\left(E S\left\|\mathbf{u}_{j}\right\|\right)^{\rho_{j}}\right)^{\delta_{j}} \\
& C_{0} D\left(h\left(\xi_{1}: \cdots: \xi_{D}\right)+\log (D)\right) .
\end{aligned}
$$

Le réel $U_{0}\left(\right.$ resp. $\left.U_{0}^{\prime}\right)$ est comparable, à une fonction polynomiale de $C_{0}$ près, à la mesure du théorème 3.1 (resp. théorème 3.2). Pour ne pas alourdir la démonstration, nous ne traiterons que le cas général avec $U_{0}$. Il va de soi que si nous sommes dans le cas particulier où $u_{0} \neq 0$ et $\prod_{j=1}^{n} \mathbf{G}_{j}$ est une variété abélienne alors $U_{0}$ doit être remplacé par $U_{0}^{\prime}$ (et de même pour les paramètres définis ci-après). Nous insistons sur le fait que la légère amélioration de la mesure d'indépendance dans le cas particulier semi-abélien n'a été rendue possible que grâce à la condition (plus faible) exigée par le lemme de zéros (voir démonstration du corollaire 6.19).

Notons alors

$$
\begin{gathered}
\widetilde{T}:=\frac{C_{0}^{4} U_{0}}{S \log E}, \quad\left(\text { resp. } \widetilde{T}:=\frac{C_{0}^{4} U_{0}^{\prime}}{S \log E}\right) \\
\widetilde{T}_{0}:=\frac{\widetilde{T}}{C_{0}^{2}}, \\
\widetilde{D}_{0}:=\frac{U_{0}}{D h(W)+D h\left(u_{0}\right)+S \log E}, \\
\left(r e s p . \quad \widetilde{D}_{0}:=\frac{U_{0}^{\prime}}{D h(W)+\max _{0 \leq s \leq(d+1) S}\left\{D h\left(s u_{0}\right)\right\}+C_{0}^{2} \log E}\right)
\end{gathered}
$$


et, pour $j \in\{1, \ldots, n\}$,

$$
\begin{gathered}
\widetilde{D}_{j}:=\frac{U_{0}}{S \log E+\max _{0 \leq s \leq(d+1) S}\left\{D h\left(s \mathbf{p}_{j}\right)\right\}+\left(E S\left\|\mathbf{u}_{j}\right\|\right)^{\rho_{j}}}, \\
\left(\text { resp. } \widetilde{D}_{j}:=\frac{U_{0}^{\prime}}{S \log E+\max _{0 \leq s \leq(d+1) S}\left\{D h\left(s \mathbf{p}_{j}\right)\right\}+\left(E S\left\|\mathbf{u}_{j}\right\|\right)^{\rho_{j}}}\right) .
\end{gathered}
$$

Afin de simplifier la présentation de la majoration du degré du sous-groupe obstructeur donnée au $\S 6.5$, nous supposerons que les nombres réels $\widetilde{D}_{1}, \ldots, \widetilde{D}_{n}$ sont rangés en ordre décroissant, i.e.

$$
\begin{aligned}
\max _{0 \leq s \leq(d+1) S}\left\{D h\left(s \mathbf{p}_{1}\right)\right\}+\left(E S\left\|\mathbf{u}_{1}\right\|\right)^{\rho_{1}} \\
\geq \max _{\substack{0 \leq s \leq(d+1) S \\
\geq}}\left\{D h\left(s \mathbf{p}_{2}\right)\right\}+\left(E S\left\|\mathbf{u}_{2}\right\|\right)^{\rho_{2}} \\
\geq \cdots
\end{aligned}
$$

On notera que les inégalités suivantes (qui nous seront utiles à chaque application du lemme de zéros) sont vérifiées :

- $\widetilde{T}_{0} \geq C_{0} \max \left\{\widetilde{D}_{1}, \ldots, \widetilde{D}_{n}\right\}$.

- $\widetilde{T}_{0} \geq C_{0} \widetilde{D}_{0}$ dans le cas général.

- $\widetilde{T}_{0} \geq C_{0} \frac{\widetilde{D}_{0}}{S_{0}}$ dans le cas où $u_{0} \neq 0$ et $\prod_{j=1}^{n} \mathbf{G}_{j}$ est une variété semi-abélienne.

Remarque 6.1. Ces paramètres ont été choisis de manière à satisfaire un système de contraintes qui apparaît lorsqu'on effectue la démonstration avec des paramètres libres. Une fois ce système établi, il s'agit de résoudre un problème d'optimisation sous contraintes (non linéaire). Nous pensons que ce choix est le meilleur possible. Nous renvoyons le lecteur à l'annexe A de 17] pour une justification précise de cette affirmation.

Soit $x \in \mathbb{R}^{+}$. Notons également $D_{i}^{\#}:=x \widetilde{D}_{i}, D_{i}:=\left[D_{i}^{\#}\right], T:=[\widetilde{T}]$ et $T_{0}:=\left[\widetilde{T}_{0}\right]$.

La proposition suivante, qui détermine le choix de $x$, s'énonce en fonction de la partie homogène de plus haut degré du polynôme de Hilbert-Samuel d'une sousvariété $V$ de $\mathbb{P}^{N_{0}} \times \cdots \times \mathbb{P}^{N_{n}}$, polynôme que nous notons $\mathscr{H}$ (voir la fin du $\S$ 囵). Nous n'aurons besoin que de propriétés immédiates de $\mathscr{H}$ (liées à sa structure de polynôme homogène) sauf au $\S$ 6.5, où l'interprétation géométrique (en terme de degrés partiels de $V$ ) de ses coefficients nous sera utile. Ce type de proposition, qui existe sous plusieurs formes légèrement différentes, est devenue classique depuis les travaux de P. Philippon \& M. Waldschmidt (voir proposition 5.12 de [25]).

Proposition 6.2. Il existe un réel $x \in] 0,1]$ tel que les conditions suivantes soient vérifiées.

(i) Pour tout sous-groupe algébrique connexe $\mathbf{G}^{\prime}$ de $\mathbf{G}_{\mathbf{L}}$ de codimension $r^{\prime}$, dont l'espace tangent à l'origine est inclus dans l'hyperplan $W$, on a :

$$
\begin{aligned}
(\widetilde{T})^{r^{\prime}-1} \times & \operatorname{card}\left(\frac{\Gamma_{\mathbf{p}}(S)+\mathbf{G}^{\prime}(\mathbf{L})}{\mathbf{G}^{\prime}(\mathbf{L})}\right) \times \mathscr{H}\left(\mathbf{G}^{\prime} ; D_{0}^{\#}, \ldots, D_{n}^{\#}\right) \\
& \geq C_{0} \mathscr{H}\left(\mathbf{G} ; D_{0}^{\#}, \ldots, D_{n}^{\#}\right) .
\end{aligned}
$$

(ii) Il existe au moins un sous-groupe algébrique algébrique connexe $\widetilde{\mathbf{G}}$ qui réalise l'égalité dans (19).

Fixons $\widetilde{\mathbf{G}}$ et notons $\widetilde{d}$ (resp. $\widetilde{r}$ ) sa dimension (resp. sa codimension dans $\mathbf{G}$ ).

Démonstration. Bien que la démonstration soit classique depuis [25], nous reproduisons la preuve afin de simplifier la vérification des théorèmes. 
Soit $\mathbf{G}^{\prime}$ un sous-groupe algébrique connexe de $\mathbf{G}_{\mathbf{L}}$; définissons

$$
A\left(\mathbf{G}^{\prime}\right):=(\widetilde{T})^{r^{\prime}-1} \operatorname{card}\left(\frac{\Gamma_{\mathbf{p}}(S)+\mathbf{G}^{\prime}(\mathbf{L})}{\mathbf{G}^{\prime}(\mathbf{L})}\right) \frac{\mathscr{H}\left(\mathbf{G}^{\prime} ; \widetilde{D}_{0}, \ldots, \widetilde{D}_{n}\right)}{C_{0} \mathscr{H}\left(\mathbf{G} ; \widetilde{D}_{0}, \ldots, \widetilde{D}_{n}\right)}
$$

et $B\left(\mathbf{G}^{\prime}\right):=A\left(\mathbf{G}^{\prime}\right)^{1 / r^{\prime}} \max \left\{1, A\left(\mathbf{G}^{\prime}\right)\right\}^{\frac{r^{\prime}-1}{r^{\prime}}}$. Comme nous avons

$$
\left(\operatorname{deg}_{\Phi} \mathbf{G}^{\prime}\right) \max _{\substack{0 \leq i_{k} \leq \delta_{k} \\ i_{0}+\cdots+i_{n}=d^{\prime}}}\left\{\widetilde{D}_{0}^{i_{0}} \ldots \widetilde{D}_{n}^{i_{n}}\right\} \geq \mathscr{H}\left(\mathbf{G}^{\prime} ; \widetilde{D}_{0}, \ldots, \widetilde{D}_{n}\right)
$$

et

$$
\mathscr{H}\left(\mathbf{G}^{\prime} ; \widetilde{D}_{0}, \ldots, \widetilde{D}_{n}\right) \geq\left(\operatorname{deg}_{\Phi} \mathbf{G}^{\prime}\right) \min _{\substack{0 \leq i_{k} \leq \delta_{k} \\ i_{0}+\cdots+i_{n}=d^{\prime}}}\left\{\widetilde{D}_{0}^{i_{0}} \cdots \widetilde{D}_{n}^{i_{n}}\right\}
$$

on en déduit

$$
C_{0} \widetilde{T} \times\left(\frac{\max _{0 \leq i \leq n}\left\{\widetilde{D}_{i}\right\}}{\widetilde{T}}\right)^{r^{\prime}} \geq \frac{\operatorname{deg}_{\Phi} \mathbf{G}^{\prime}}{A\left(\mathbf{G}^{\prime}\right)} \geq \frac{C_{0} \widetilde{T}}{S+1} \times\left(\frac{\max _{0 \leq i \leq n}\left\{\widetilde{D}_{i}\right\}}{\widetilde{T}}\right)^{r^{\prime}} .
$$

Ainsi, à degré (de $\mathbf{G}^{\prime}$ ) fixé, la quantité $A\left(\mathbf{G}^{\prime}\right)$ (et donc aussi $B\left(\mathbf{G}^{\prime}\right)$ ) ne prend qu'un nombre fini de valeur et lorsque $\operatorname{deg}_{\Phi} \mathbf{G}^{\prime}$ s'accroît indéfiniment, il en est de même de $A\left(\mathbf{G}^{\prime}\right)$. Ces observations justifient l'existence d'un sous-groupe connexe $\widetilde{\mathbf{G}}$ de $\mathbf{G}_{\mathbf{L}}$ tel que $B(\widetilde{\mathbf{G}})$ soit minimal parmi tous les $\mathbf{G}^{\prime}$ d'espace tangent (à l'origine) inclus dans $W$. Posons alors $x:=B(\widetilde{\mathbf{G}})$ (clairement non nul).

Montrons que $x \leq 1$.

Le choix des paramètres permet de calculer

$$
\begin{aligned}
A(\{0\}) & =\frac{(\widetilde{T})^{d} \operatorname{card}\left(\Gamma_{\mathbf{p}}(S)\right)}{C_{0}\left(\operatorname{deg}_{\Phi} \mathbf{G}\right) \widetilde{D}_{0}^{\delta_{0}} \cdots \widetilde{D}_{n}^{\delta_{n}}} \\
& =\frac{1}{\operatorname{deg}_{\Phi} \mathbf{G}}
\end{aligned}
$$

et, donc, $1 \geq A(\{0\})$, ce qui implique

$$
x=\min _{t_{\mathbf{G}^{\prime}} \subseteq W}\left\{B\left(\mathbf{G}^{\prime}\right)\right\} \leq B(\{0\})=A(\{0\})^{1 /(d+1)} \leq 1 .
$$

Montrons que l'inégalité (19) est vérifiée :

Posons

$$
\aleph_{\mathbf{G}^{\prime}}=(\widetilde{T})^{r^{\prime}-1} \operatorname{card}\left(\frac{\Gamma_{\mathbf{p}}(S)+\mathbf{G}^{\prime}(\mathbf{L})}{\mathbf{G}^{\prime}(\mathbf{L})}\right) \frac{\mathscr{H}\left(\mathbf{G}^{\prime} ; D_{0}^{\#}, \ldots, D_{n}^{\#}\right)}{C_{0} \mathscr{H}\left(\mathbf{G} ; D_{0}^{\#}, \ldots, D_{n}^{\#}\right)} .
$$

On a $A\left(\mathbf{G}^{\prime}\right)=x^{r^{\prime}} \aleph_{\mathbf{G}^{\prime}}$.

- Si $A\left(\mathbf{G}^{\prime}\right) \geq 1$, on a $\aleph_{\mathbf{G}^{\prime}} \geq \frac{1}{x^{r^{\prime}}} \geq 1$.

- Si $A\left(\mathbf{G}^{\prime}\right)<1$, on a $B\left(\mathbf{G}^{\prime}\right)=A\left(\mathbf{G}^{\prime}\right)^{1 / r^{\prime}} \geq x$, donc $\left(\aleph_{\mathbf{G}^{\prime}}\right)^{1 / r^{\prime}} x \geq x$, i.e. $\aleph_{\mathbf{G}^{\prime}} \geq 1$. En remplaçant les inégalités par des égalités $(x=B(\widetilde{\mathbf{G}})$ et nécessairement $A(\widetilde{\mathbf{G}}) \leq 1$ sinon $x=A(\widetilde{\mathbf{G}})$ ce qui contredit $x \leq 1)$, on obtient $\aleph_{\widetilde{\mathbf{G}}}=1$.

Tout au long de la démonstration, nous aurons à tenir compte du sous-groupe $\widetilde{\mathbf{G}}$ introduit dans cette proposition. Nous montrerons plus loin (corollaire 6.19) que le choix de $x$ permet d'assurer qu'aucun des entiers $D_{0}, \ldots, D_{n}$ n'est nul.

Définition 6.3. Nous dirons que nous sommes dans le cas périodique s'il existe $s \in\{1, \ldots, 2(d+1) S\}$ tel que $s \mathbf{u} \in \Omega_{\mathbf{G}(\mathbb{C})}+t_{\widetilde{\mathbf{G}}}(\mathbb{C})$, et dans le cas non-périodique si un tel entier $s$ n'existe pas.

Le cas non-périodique se prêtera à une extrapolation sur les points et nous poserons

$$
\Upsilon:=\left\{(\underline{t}, s) \in \mathbb{N}^{d} \times \mathbb{N} ;|\underline{t}| \leq 2(d+1) T \text { et } 0 \leq s<S_{0}\right\}
$$


Dans le cas périodique, nous extrapolerons sur les dérivées et nous poserons

$$
\Upsilon:=\left\{(\underline{t}, s) \in \mathbb{N}^{d} \times \mathbb{N} ;|\underline{t}| \leq 2(d+1) T, t_{d}<T_{0} \text { et } 0 \leq s<2(d+1) S\right\} .
$$

Le cardinal de $\Upsilon$ résulte du calcul élémentaire suivant.

Lemme 6.4. Si $n, A, B$ sont des entiers naturels non nuls alors le cardinal de l'ensemble

$$
\left\{\left(x_{1}, \ldots, x_{n}\right) \in \mathbb{N}^{n} ; x_{n} \leq A \text { et } x_{1}+\cdots+x_{n} \leq A+B\right\}
$$

est égal ̀̀ $\left(\begin{array}{c}A+B+n \\ n\end{array}\right)-\left(\begin{array}{c}B-1+n \\ n\end{array}\right)$.

Nous en déduisons alors que

$$
\operatorname{card} \Upsilon=\left(\begin{array}{c}
2(d+1) T+d \\
d
\end{array}\right) \times S_{0}
$$

dans le cas non-périodique et

$$
\operatorname{card} \Upsilon=(2(d+1) S-1) \times\left[\left(\begin{array}{c}
2(d+1) T+d \\
d
\end{array}\right)-\left(\begin{array}{c}
2(d+1) T-T_{0}+d+1 \\
d
\end{array}\right)+1\right]
$$

dans le cas périodique. En réalité, nous aurons seulement besoin de la majoration (assez grossière)

$$
\mu:=\operatorname{card} \Upsilon \leq 2(d+1) S(2(d+1) T+1)^{d} \leq c_{14} S T^{d} \leq e^{c_{15} U_{0}} .
$$

Les dérivées que nous considérerons seront toutes dans la direction de l'hyperplan $W$. Cependant, dans le cas périodique, nous choisirons une direction privilégiée (transcendante), selon laquelle nous extrapolerons.

6.3. Choix de bases pour l'hyperplan $W$. Dans ce paragraphe, nous fixons une base orthonormée de l'hyperplan $W$, utile pour l'extrapolation ii selon les dérivées ¿i. dans le cas périodique.

- Par définition, dans la base $\left(e_{0}, \ldots, e_{d}\right)$ de $t_{\mathbf{G}}$, l'hyperplan $W$ a pour équation $z_{0}=\beta_{1} z_{1}+\cdots+\beta_{d} z_{d}$. La famille $\left\{\widetilde{e}_{i}=e_{i}+\beta_{i} e_{0}\right\}_{1 \leq i \leq d}$ constitue donc une base $\widetilde{\mathbf{e}}$ de $W$.

- L'isomorphisme $\left(z_{1}, \ldots, z_{d}\right) \rightarrow z_{1} \widetilde{e}_{1}+\cdots+z_{d} \widetilde{e}_{d}$, entre $\mathbb{C}^{d}$ et $W \otimes \mathbb{C}($ le produit tensoriel, ici, est relatif au plongement initial $\sigma_{0}$ de $\mathbf{L}$ dans $\mathbb{C}$ ), munit $W \otimes \mathbb{C}$ d'une (unique) structure hermitienne, héritée du produit scalaire canonique sur $\mathbb{C}^{d}$. Nous noterons |.| la norme ainsi obtenue ${ }^{7}$ sur $W$, et nous poserons $\mathbf{e}^{\prime}:=\left(e_{1}^{\prime}, \ldots, e_{d}^{\prime}\right)$ une base orthonormée de $W$ pour ce produit scalaire, telle que $\left(e_{1}^{\prime}, \ldots, e_{\widetilde{d}}^{\prime}\right)$ soit également une base orthonormée de $t_{\widetilde{\mathbf{G}}}(\mathbb{C})$. Nous supposerons de plus que $\left(e_{\widetilde{d}+1}^{\prime}, \ldots, e_{d}^{\prime}\right)$ est ordonnée de telle façon que si $\mathbf{w}:=u_{1} \widetilde{e}_{1}+\cdots+u_{d} \widetilde{e}_{d}$ s'écrit $w_{1} e_{1}^{\prime}+\cdots+w_{d} e_{d}^{\prime}$ dans la base $\mathbf{e}^{\prime}$, alors

$$
\left|w_{d}\right|=\max \left\{\left|w_{i}\right| ; \quad \widetilde{d}+1 \leq i \leq d\right\} .
$$

Comme les bases $\widetilde{\mathbf{e}}$ et $\mathbf{e}^{\prime}$ de $W$ sont orthonormées pour le même produit hermitien sur $W \otimes \mathbb{C}$, les matrices de passages entre elles sont unitaires. On notera également que $\mathbf{u}-\mathbf{w}=\Lambda . e_{0}$ et donc $\|\mathbf{u}-\mathbf{w}\|=|\Lambda|$.

6.4. Le cas périodique : compléments. Grâce au résultat principal de [5], nous obtenons la

Proposition 6.5. Il existe une constante $c_{16}$, ne dépendant que de $(\mathbf{G}, \Phi, \mathbf{e})$, telle que, si $\omega \in \Omega_{\mathbf{G}(\mathbb{C})} \backslash \Omega_{\widetilde{\mathbf{G}}(\mathbb{C})}$, on ait

$$
\mathrm{d}\left(\omega, t_{\widetilde{\mathbf{G}}}(\mathbb{C})\right) \geq \frac{1}{c_{16} \operatorname{deg}_{\Phi} \pi(\widetilde{\mathbf{G}})}
$$

où $\pi: \mathbf{G}(\mathbb{C}) \rightarrow \mathbf{G}_{1}(\mathbb{C}) \times \cdots \times \mathbf{G}_{n}(\mathbb{C})$ est la projection canonique.

\footnotetext{
${ }^{7}$ Dans le contexte, il ne devrait pas y avoir de confusion avec une ii simple i. $i$ valeur absolue.
} 
Démonstration. Comme $\Omega_{\mathbb{G}_{a}(\mathbb{C})}=\{0\}$, on a $\omega=\pi^{\prime}(\omega)$ où $\pi^{\prime}$ est la projection de

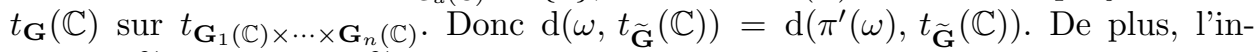
clusion $\widetilde{\mathbf{G}} \subseteq \mathbb{G}_{\mathrm{a}} \times \pi(\widetilde{\mathbf{G}})$ implique $t_{\widetilde{\mathbf{G}}} \subseteq t_{\mathbb{G}_{\mathrm{a}}} \oplus t_{\pi(\widetilde{\mathbf{G}})} \operatorname{donc} \mathrm{d}\left(\pi^{\prime}(\omega), t_{\widetilde{\mathbf{G}}}(\mathbb{C})\right) \geq$ $\mathrm{d}\left(\pi^{\prime}(\omega), t_{\pi(\widetilde{\mathbf{G}})}(\mathbb{C})\right)$. D'après le corollaire 2 de 河, on a

$$
\mathrm{d}\left(\pi^{\prime}(\omega), t_{\pi(\widetilde{\mathbf{G}})}(\mathbb{C})\right) \geq \frac{1}{c_{16} \operatorname{deg}_{\Phi} \pi(\widetilde{\mathbf{G}})},
$$

ce qui conclut la démonstration.

Des propriétés supplémentaires du groupe $\widetilde{\mathbf{G}}$, introduit au $\S 6.2$, seront obtenues à l'aide de cette proposition. Mais auparavant nous voulons majorer le degré de $\pi(\widetilde{\mathbf{G}})$.

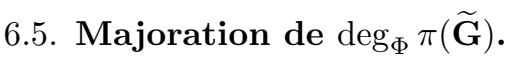

Proposition 6.6. Soit $\mathscr{H}$ le polynôme multi-homogène (de Hilbert-Samuel) de G. Alors on a

$$
\mathscr{H}\left(\widetilde{\mathbf{G}} ; x_{0}, \ldots, x_{n}\right) \geq\left(\operatorname{deg}_{\Phi} \pi(\widetilde{\mathbf{G}})\right) \min \left\{x_{i_{1}} \cdots x_{i_{\tilde{d}}}\right\}
$$

où $\widetilde{d}=\operatorname{dim} \widetilde{\mathbf{G}}$ et le minimum porte sur les $\widetilde{d}$-uplets $\left(i_{1}, \ldots, i_{\widetilde{d}}\right)$ vérifiant $1 \leq i_{1} \leq$ $\cdots \leq i_{\tilde{d}} \leq n$ et chaque $i_{\ell}$ apparaissant au plus $\delta_{i_{\ell}}$ fois.

Remarque 6.7. L'hypothèse $t_{\widetilde{\mathbf{G}}} \subseteq W$ implique $\operatorname{dim} \pi(\widetilde{\mathbf{G}})=\widetilde{d}$ car la surjection $\widetilde{\mathbf{G}} \rightarrow \pi(\widetilde{\mathbf{G}})$ donne $\operatorname{dim} \pi(\widetilde{\mathbf{G}}) \leq \operatorname{dim} \widetilde{\mathbf{G}}$, et si cette inégalité était stricte, l'inclusion $\widetilde{\mathbf{G}} \subseteq \mathbf{G}_{0} \times \pi(\widetilde{\mathbf{G}})$ entrainerait $t_{\widetilde{\mathbf{G}}}=t_{\mathbf{G}_{0} \times \pi(\widetilde{\mathbf{G}})}=t_{\mathbf{G}_{0}} \oplus t_{\pi(\widetilde{\mathbf{G}})}$, et donc $t_{\mathbf{G}_{0}} \subseteq W$ ce qui est faux.

Démonstration. C'est immédiat d'après l'interprétation géométrique des coefficients du polynôme de Hilbert-Samuel (voir [23, 24]).

Lemme 6.8. Soit $m$ le plus grand entier de $\{1, \ldots, n\}$ tel que $\delta_{1}+\cdots+\delta_{m-1} \leq \widetilde{d}$. Posons $\delta_{m}^{\prime}:=\widetilde{d}-\delta_{1}-\cdots-\delta_{m-1}$. Alors, le degré $\operatorname{deg}_{\Phi} \pi(\widetilde{\mathbf{G}})$ est majoré par

$$
\begin{aligned}
& C_{0}^{4 d+2}\left(\operatorname{deg}_{\Phi} \mathbf{G}\right) \frac{\operatorname{card}\left(\Gamma_{\mathbf{p}}(\mathfrak{a})\right)}{(\mathfrak{a} \log E)^{\tilde{d}}} \times \max \left\{1, \frac{D h\left(\xi_{1}: \cdots: \xi_{D}\right)+D \log (D)}{U}\right\} \\
& \times \prod_{\ell=1}^{m-1}\left(\mathfrak{a} \log E+\max _{0 \leq s \leq(d+1) C_{0}^{5 \mathfrak{a}}}\left\{D h\left(s \mathbf{p}_{\ell}\right)\right\}+\left(E \mathfrak{a}\left\|\mathbf{u}_{\ell}\right\|\right)^{\rho_{\ell}}\right)^{\delta_{\ell}} \\
& \times\left(\mathfrak{a} \log E+\max _{0 \leq s \leq(d+1) C_{0}^{5} \mathfrak{a}}\left\{D h\left(s \mathbf{p}_{m}\right)\right\}+\left(E \mathfrak{a}\left\|\mathbf{u}_{m}\right\|\right)^{\rho_{m}}\right)^{\delta_{m}^{\prime}} .
\end{aligned}
$$

\section{Remarques :}

$x$ Cette inégalité précise la majoration (4) du théorème 3.1 en montrant l'origine des entiers $j_{1}, \ldots, j_{m}$ via l'hypothèse (18), p. 16 .

$x$ En réalité, pour la démonstration du théorème 3.1, nous utiliserons plus couramment la majoration plus faible :

$$
\begin{aligned}
& \operatorname{deg}_{\Phi} \pi(\widetilde{\mathbf{G}}) \\
& \leq C_{0}^{20 d}\left(D+D h\left(\mathbf{p}_{1}\right)+\cdots+D h\left(\mathbf{p}_{n}\right)+E\left\|\mathbf{u}_{1}\right\|+\cdots+E\left\|\mathbf{u}_{n}\right\|\right)^{20 d}
\end{aligned}
$$

car c'est le logarithme de $\operatorname{deg}_{\Phi} \pi(\widetilde{\mathbf{G}})$ qui apparaîtra. 
du lemme 6.8. La proposition 6.6 et la définition de $\widetilde{\mathbf{G}}$ entrainent

$$
\begin{aligned}
\frac{C_{0}}{(\widetilde{T})^{\tilde{r}-1} \operatorname{card}\left(\frac{\Gamma_{\mathbf{p}}(S)+\widetilde{\mathbf{G}}(\mathbf{L})}{\mathbf{G}(\mathbf{L})}\right)} & =\frac{\mathscr{H}\left(\widetilde{\mathbf{G}} ; D_{0}^{\#}, \ldots, D_{n}^{\#}\right)}{\mathscr{H}\left(\mathbf{G} ; D_{0}^{\#}, \ldots, D_{n}^{\#}\right)} \\
& \geq \frac{\operatorname{deg}_{\Phi} \pi(\widetilde{\mathbf{G}})}{\operatorname{deg}_{\Phi} \mathbf{G}} \frac{1}{D_{0}^{\#} \max \left\{D_{j_{1}}^{\#} \cdots D_{j_{\tilde{r}-1}}^{\#}\right\}} \\
& =\frac{\operatorname{deg}_{\Phi} \pi(\widetilde{\mathbf{G}})}{\operatorname{deg}_{\Phi} \mathbf{G}} \frac{1}{D_{0}^{\#}\left(D_{n}^{\#}\right)^{\delta_{n}} \cdots\left(D_{\ell+1}^{\#}\right)^{\delta_{m+1}}\left(D_{\ell}^{\#}\right)^{\delta_{m}-\delta_{m}^{\prime}}}
\end{aligned}
$$

où $m$ est l'entier défini dans l'énoncé du lemme. Il s'ensuit

$$
\operatorname{deg}_{\Phi} \pi(\widetilde{\mathbf{G}}) \leq C_{0}\left(\operatorname{deg}_{\Phi} \mathbf{G}\right) \widetilde{D}_{0} \frac{\widetilde{D}_{n}^{\delta_{n}} \cdots \widetilde{D}_{m}^{\delta_{m}-\delta_{m}^{\prime}}}{\widetilde{T}^{\tilde{r}-1}},
$$

ce qui, en remplaçant les paramètres par leur valeur, implique le résultat voulu. La seconde remarque qui suit le lemme est claire. Le point important (pour l'inégalité (价) du théorème 3.1 mais, aussi, et surtout, pour le théorème 1.2) est que la majoration de $\operatorname{deg}_{\Phi} \pi(\widetilde{\mathbf{G}})$ soit indépendante de $h(W)$.

Corollaire 6.9. Dans le cas périodique, supposons $\mathbf{u} \notin t_{\widetilde{\mathbf{G}}}(\mathbb{C})$. Alors, pour $C_{0}$ assez grand, la distance $\mathrm{d}\left(\mathbf{u}, t_{\widetilde{\mathbf{G}}}(\mathbb{C})\right)$ est minorée par :

$$
C_{0}^{-21 d}\left(D+D\left(h\left(\mathbf{p}_{1}\right)+\cdots+h\left(\mathbf{p}_{n}\right)\right)+E\left\|\mathbf{u}_{1}\right\|+\cdots+E\left\|\mathbf{u}_{n}\right\|\right)^{-20 d} .
$$

Et, toujours dans le cas périodique, supposons maintenant que

$$
|\Lambda|<\frac{1}{2} \mathrm{~d}\left(\mathbf{u}, t_{\widetilde{\mathbf{G}}}(\mathbb{C})\right) .
$$

Alors $\mathbf{w} \notin t_{\widetilde{\mathbf{G}}}(\mathbb{C})$ et $t_{\widetilde{\mathbf{G}}}(\mathbb{C}) \subsetneq W \otimes \mathbb{C}$. En particulier $w_{d} \neq 0$.

Remarque 6.10. Notons que l'hypothèse (27) implique que $\mathbf{u} \notin t_{\widetilde{\mathbf{G}}}(\mathbb{C})$.

Démonstration. Soit $s_{0} \in\{1, \ldots, 2(d+1) S\}$ minimal tel que $s_{0} \mathbf{u} \in \Omega_{\mathbf{G}(\mathbb{C})}+t_{\widetilde{\mathbf{G}}}(\mathbb{C})$. On a donc $s_{0} \leq 2(d+1) \operatorname{card}\left(\frac{\Gamma_{\mathbf{p}}(S)+\widetilde{\mathbf{G}}(\mathbf{L})}{\widetilde{\mathbf{G}}(\mathbf{L})}\right)$. Comme $s_{0} \mathbf{u} \notin \Omega_{\widetilde{\mathbf{G}}(\mathbb{C})}\left(\operatorname{sinon} \mathbf{u} \in t_{\widetilde{\mathbf{G}}}(\mathbb{C})\right)$, la proposition 6.5 et la majoration (25) impliquent l'inégalité voulue.

Par ailleurs, si w appartenait à $t_{\widetilde{\mathbf{G}}}(\mathbb{C})$, nous aurions $|\Lambda|=\|\mathbf{u}-\mathbf{w}\| \geq \mathrm{d}\left(\mathbf{u}, t_{\widetilde{\mathbf{G}}}(\mathbb{C})\right)$ ce qui contredit l'hypothèse $(27)$. Comme $\mathbf{w} \in(W \otimes \mathbb{C}) \backslash t_{\widetilde{\mathbf{G}}}(\mathbb{C})$, on a $t_{\widetilde{\mathbf{G}}}(\mathbb{C}) \subsetneq W \otimes \mathbb{C}$, et par définition de $w_{d}(\S 6.3)$, on a $w_{d} \neq 0$.

Remarque 6.11. Nous avons supposé $\mathbf{u} \notin t_{\widetilde{\mathbf{G}}}(\mathbb{C})$ alors qu'il serait plus ii naturel ii de se contenter de $\mathbf{u} \notin W \otimes \mathbb{C}$. Mais, si l'hypothèse du corollaire 6.9 est fausse, nous disposons d'une précision supplémentaire sur $\mathbf{u}$, qui constitue la première partie du théorème 3.1 (autrement dit, le théorème du sous-groupe analytique de Wüstholz).

6.6. Nouvelle base pour $W \otimes \mathbb{C}$. Dans le cas périodique, nous posons

$$
\mathbf{f}:=\left(e_{1}^{\prime}, \ldots, e_{d-1}^{\prime}, \mathbf{w}\right)
$$

(es $\mathbf{e}^{\prime}$ est la base orthonormée définie au $\S\left[6.3\right.$ ). C'est une base de $W \otimes \mathbb{C}$ car $w_{d} \neq$ 0 . Pour unifier les notations, nous noterons également $\mathbf{f}:=\mathbf{e}^{\prime}$ dans le cas nonpériodique.

Lemme 6.12 (Lemme 4.16 de $[19]$ ). Pour tout $x \in W$, on $a|x| \leq\|x\| \leq$ $(\sqrt{d+1})|x|$.

Démonstration. Soit $x \in W$. On écrit

$$
\begin{aligned}
x & =x_{1} \widetilde{e}_{1}+\cdots+x_{d} \widetilde{e}_{d} \\
& =\left(\beta_{1} x_{1}+\cdots+\beta_{d} x_{d}\right) e_{0}+x_{1} e_{1}+\cdots+x_{d} e_{d}
\end{aligned}
$$

On a alors $|x|^{2}=\left|x_{1}\right|^{2}+\cdots+\left|x_{d}\right|^{2}$ et $\|x\|^{2}=\left|x_{1}\right|^{2}+\cdots+\left|x_{d}\right|^{2}+\left|\beta_{1} x_{1}+\cdots+\beta_{d} x_{d}\right|^{2}$.

Donc $|x| \leq\|x\|$ résulte des définitions et $\|x\| \leq(\sqrt{d+1})|x|$ vient du fait que les $\beta_{i}$ sont de module $\leq 1$, et de l'inégalité de Cauchy-Schwarz. 
En procédant comme N. HiRATA-Kohno dans [19] (pp. 418 - 419), nous en déduisons alors la

Proposition 6.13 (Variante de la proposition 4.17 de $[19]$ ). Dans le cas périodique, supposons que le nombre $|\Lambda|$ soit strictement inférieur $\grave{a} \frac{1}{2} \mathrm{~d}\left(\mathbf{u}, t_{\widetilde{\mathbf{G}}}(\mathbb{C})\right)$. Alors on a

$$
\left|w_{d}\right| \geq \frac{1}{2(d+1)} \mathrm{d}\left(\mathbf{u}, t_{\widetilde{\mathbf{G}}}(\mathbb{C})\right) .
$$

Démonstration. Posons $x:=w_{1} e_{1}^{\prime}+\cdots+w_{\tilde{d}} e_{\widetilde{d}}^{\prime}$. C'est un élément de $t_{\widetilde{\mathbf{G}}}(\mathbb{C})$ et $\mathbf{w}-x=$ $w_{\widetilde{d}+1} e_{\widetilde{d}+1}^{\prime}+\cdots+w_{d} e_{d}^{\prime}$. Donc $|\mathbf{w}-x| \leq(\sqrt{d-\widetilde{d}})\left|w_{d}\right| \leq(\sqrt{d+1})\left|w_{d}\right|$ car $\left|w_{d}\right|=\max _{\widetilde{d} \leq i \leq d}\left|w_{i}\right|$. Alors $\|\mathbf{w}-x\| \leq(d+1)\left|w_{d}\right|$ en vertu du lemme 6.12, puis $\|\mathbf{u}-x\|-\|\mathbf{w}-\mathbf{u}\| \leq(d+1)\left|w_{d}\right|$, ce qui implique $\mathrm{d}\left(\mathbf{u}, t_{\widetilde{\mathbf{G}}}(\mathbb{C})\right)-|\Lambda| \leq(d+1)\left|w_{d}\right|$. L'hypothèse permet de conclure.

Du corollaire 6.9 et de la proposition 6.13, nous déduisons le

Corollaire 6.14. Sous les hypothèses du corollaire 6.9, la valeur absolue de $w_{d}$ est minorée par

$$
C_{0}^{-22 d}\left(D+D\left(h\left(\mathbf{p}_{1}\right)+\cdots+h\left(\mathbf{p}_{n}\right)\right)+E\left\|\mathbf{u}_{1}\right\|+\cdots+E\left\|\mathbf{u}_{n}\right\|\right)^{-20 d} .
$$

6.7. Changement de bases de dérivations. L'objectif de ce paragraphe est de comparer les dérivées le long de $W$ d'une fonction analytique, lorsqu'on change la base de $W \otimes \mathbb{C}$, et de préciser le résultat dans les cas périodique et non-périodique.

Si $\mathbf{v}=\left(v_{1}, \ldots, v_{n}\right)$ est un élément de $\mathbb{C}^{n}$, nous désignons par $\mathcal{D}_{\mathbf{v}}$ la dérivation

$$
\mathcal{D}_{\mathbf{v}}:=v_{1} \frac{\partial}{\partial z_{1}}+\cdots+v_{n} \frac{\partial}{\partial z_{n}} .
$$

Si nous considérons $\underline{t} \in \mathbb{N}^{g}$ et $\mathbf{e}=\left\{\mathbf{e}_{1}, \ldots, \mathbf{e}_{g}\right\}$ une famille d'éléments de $\mathbb{C}^{n}$, nous notons

$$
\mathcal{D} \frac{t}{\mathbf{e}}:=\mathcal{D}_{\mathbf{e}_{1}}^{t_{1}} \cdots \mathcal{D}_{\mathbf{e}_{g}}^{t_{g}} .
$$

Comme dans les articles [13, 14], nous travaillerons dans la suite avec des dérivées divisées. Nous aurons besoin de la variante suivante du lemme 3.1 de [25, qui ne comportait pas de factorielles.

Lemme 6.15 (Variante du lemme 3.1 de 25]). Soient $\left(\mathrm{a}_{i, j}\right)_{\substack{1 \leq i \leq n \\ 1 \leq j \leq m}}$ des nombres complexes, $\mathrm{f}_{1}, \ldots, \mathrm{f}_{m}, z$ des vecteurs de $\mathbb{C}^{d+1}$, et $\Theta$ une fonction analytique complexe au voisinage de $z$. Posons $\mathrm{e}_{i}=\sum_{j=1}^{m} \mathrm{a}_{i, j} \mathrm{f}_{j}$ et $\mathrm{A}:=\sum_{i, j}\left|\mathrm{a}_{i, j}\right|$. Alors, pour tout entier naturel $T$, on a

$$
\max _{|\underline{\mid}|=T}\left\{\left|\frac{\mathcal{D}_{\mathrm{e}_{1}}^{t_{1}} \cdots \mathcal{D}_{\mathrm{e}_{n}}^{t_{n}}}{\underline{t} !} \Theta(z)\right|\right\} \leq \mathrm{A}^{T} \times \max _{|\underline{h}|=T}\left\{\left|\frac{\mathcal{D}_{\mathrm{f}_{1}}^{h_{1}} \cdots \mathcal{D}_{\mathrm{f}_{m}}^{h_{m}}}{\underline{h} !} \Theta(z)\right|\right\} .
$$

Démonstration. La démonstration consiste à développer $\frac{1}{\underline{t} !} \mathcal{D}_{\mathrm{e}_{1}}^{t_{1}} \cdots \mathcal{D}_{\mathrm{e}_{n}}^{t_{n}}$ en utilisant la formule du multinôme :

$$
\begin{aligned}
& \frac{1}{\underline{t} !} \mathcal{D}_{\mathrm{e}_{1}}^{t_{1}} \cdots \mathcal{D}_{\mathrm{e}_{n}}^{t_{n}} \Theta(z)= \\
& \sum_{\substack{t_{i, 1}+\cdots+t_{i, m}=t_{i} \\
1 \leq i \leq n}}\left(\prod_{i, j} \frac{\left(\mathrm{a}_{i, j}\right)^{t_{i, j}}}{\left(t_{i, j}\right) !}\right)\left\{\mathcal{D}_{\mathrm{f}_{1}}^{t_{1,1}+\cdots+t_{n, 1}} \cdots \mathcal{D}_{\mathrm{f}_{m}}^{t_{1, m}+\cdots+t_{n, m}} \Theta\right\}(z) .
\end{aligned}
$$

Le lemme se déduit alors des majorations

et

$$
\prod_{j=1}^{m}\left(t_{1, j}+\cdots+t_{n, j}\right) ! \leq\left(\sum_{i, j} t_{i, j}\right) !
$$

$$
\sum_{t_{i, 1}+\cdots+t_{i, m}=t_{i}}\left\{\left(\prod_{i, j} \frac{\left|\mathbf{a}_{i, j}\right|^{t_{i, j}}}{\left(t_{i, j}\right) !}\right) \times\left(\sum_{i, j} t_{i, j}\right) !\right\} \leq\left(\sum_{i, j}\left|\mathbf{a}_{i, j}\right|\right)^{|\underline{\underline{t}}|} .
$$


Grâce à ce lemme appliqué aux bases f et $\widetilde{\mathbf{e}}$ de $W$ construites précédemment, nous obtenons la

Proposition 6.16. Soit $\Theta$ une fonction analytique au voisinage d'un point $z \in$ $\mathbb{C}^{d+1}$. Soit $m$ un entier naturel.

- Dans le cas non-périodique:

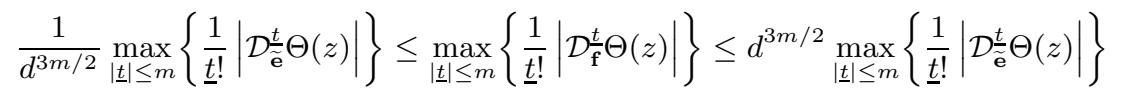

- Dans le cas périodique :

$$
\max _{|\underline{t}| \leq m}\left\{\frac{1}{\underline{t} !}|\mathcal{D} \underline{\tilde{\mathbf{e}}} \Theta(z)|\right\} \leq d^{m / 2}\left(d+\frac{1+\left|w_{1}\right|+\cdots+\left|w_{d-1}\right|}{\left|w_{d}\right|}\right)^{m} \max _{|\underline{t}| \leq m}\left\{\frac{1}{\underline{t} !}\left|\mathcal{D} \frac{t}{\mathbf{f}} \Theta(z)\right|\right\}
$$

$$
\max _{\mid \underline{|\underline{t}| \leq m}}\left\{\frac{1}{\underline{\underline{t}} !}\left|\mathcal{D} \frac{\underline{t}}{\mathbf{f}} \Theta(z)\right|\right\} \leq d^{m / 2}(d+\|\mathbf{u}\|)^{m} \max _{|\underline{t}| \leq m}\left\{\frac{1}{\underline{t} !}|\mathcal{D} \underline{\tilde{\mathbf{e}}} \Theta(z)|\right\} .
$$

Remarque 6.17. Dans le cas périodique, on a :

$$
\begin{aligned}
& \frac{1+\left|w_{1}\right|+\cdots+\left|w_{d-1}\right|}{\left|w_{d}\right|} \\
& \leq 2(d+1)\left(\frac{\sqrt{d}}{2}+\frac{1+d^{1 / 2}\|\mathbf{u}\|}{\mathrm{d}\left(\mathbf{u}, t_{\widetilde{\mathbf{G}}}(\mathbb{C})\right)}\right) \\
& \leq C_{0}^{23 d}\left(D+D h\left(\mathbf{p}_{1}\right)+\cdots+D h\left(\mathbf{p}_{n}\right)+E\left\|\mathbf{u}_{1}\right\|+\cdots+E\left\|\mathbf{u}_{n}\right\|\right)^{20 d}
\end{aligned}
$$

pour $C_{0}$ assez grand. C'est cette inégalité qui requiert un majorant de $\operatorname{deg} \pi(\widetilde{\mathbf{G}})$ indépendant de $h(W)$, sans quoi l'inégalité (29) réintroduirait un terme en

$$
T \times \log (\text { d'une fonction de } h(W)) .
$$

La dernière inégalité résulte directement du corollaire 6.9. Pour la première, on écrit

$$
\begin{aligned}
1+\left|w_{1}\right|+\cdots+\left|w_{d-1}\right| & \leq 1+d^{1 / 2}|\mathbf{w}| \\
& \leq 1+d^{1 / 2}\|\mathbf{w}\| \\
& \leq 1+d^{1 / 2}(\|\mathbf{u}\|+|\Lambda|) \\
& \leq 1+d^{1 / 2}\left(\|\mathbf{u}\|+\frac{1}{2} \mathrm{~d}\left(\mathbf{u}, t_{\widetilde{\mathbf{G}}}(\mathbb{C})\right)\right)
\end{aligned}
$$

et on utilise la minoration $\left|w_{d}\right| \geq \frac{1}{2(d+1)} \mathrm{d}\left(\mathbf{u}, t_{\widetilde{\mathbf{G}}}(\mathbb{C})\right)$ (proposition 6.13).

6.8. Rang du système linéaire. Soit $P$ un polynôme multihomogène de $\mathbb{P}(=$ $\left.\mathbb{P}^{N_{0}} \times \cdots \times \mathbb{P}^{N_{n}}\right)$, à coefficients dans $\mathbf{L}$, de multidegré $\underline{D}:=\left(D_{0}, \ldots, D_{n}\right)$ (les entiers $D_{i}$ sont ceux définis au $\S$ 6.2) et qui ne s'annule pas identiquement sur $\mathbf{G}$. L'application $\Phi=\left(\Phi_{0}, \ldots, \Phi_{n}\right)$, définie p. 14, est une application holomorphe de $t_{\mathbf{G}}(\mathbb{C})$ dans $\mathbb{C}^{\sum_{i=0}^{n}\left(N_{i}+1\right)} ;$ posons

$$
F:=P \circ \Phi .
$$

Nous nous intéressons au rang du système linéaire — où les coordonnées des coefficients de $P$ dans la base de $\mathbf{L}$ sont, ici, vus comme des inconnues — défini par les conditions d'annulation suivantes :

$$
\forall(\underline{t}, s) \in \Upsilon, \quad \mathcal{D} \underline{\underline{t}} F(s \mathbf{u})=0 .
$$

Soit $E_{\mathbb{C}}:=(\mathbb{C}[\mathbb{P}] / \mathcal{I}(\mathbf{G}))_{D}$ l'ensemble des polynômes $P$, comme ci-dessus, à coefficients complexes $(\mathcal{I}(\mathbf{G})$ est l'idéal annulateur de $\mathbf{G}(\mathbb{C})$ dans $\mathbb{C}[\mathbb{P}])$. L'ensemble des classes des monômes $\mathbf{X} \underline{\lambda}$ de $\mathbb{C}[\mathbb{P}]_{\underline{D}}$ (polynômes multihomogènes de multidegrés $\underline{D}$ à coefficients complexes) forme une famille génératrice de $E_{\mathbb{C}}$. Soit $\mathcal{B}$ une famille de tels monômes dont les classes forment une base de $E_{\mathbb{C}}$. Le polynôme $P$ peut s'écrire

$$
\sum_{\underline{\lambda}, i} a_{\underline{\lambda}, i} \xi_{i} \mathbf{X}^{\underline{\lambda}}
$$


où $a_{\underline{\lambda}, i} \in \mathbb{Q}$ et $\mathbf{X}^{\underline{\lambda}} \in \mathcal{B}$. Les inconnues du système (31) seront les $a_{\underline{\lambda}, i}$, qui sont au nombre de $\nu:=D \operatorname{dim} E_{\mathbb{C}}$. On peut noter que $E_{\mathbb{C}} \neq\{0\}$, i.e. $\left(D_{0}, \ldots, D_{n}\right) \neq$ $(0, \ldots, 0)$ comme on le voit aisément à partir de la minoration $x \geq A(\widetilde{\mathbf{G}})$ déduite de la définition de $x$ (p. 17).

Lemme 6.18. Soit $\rho$ le rang du système (31). Il existe une constante $c_{17}$, ne dépendant que de $(\mathbf{G}, \Phi, \mathbf{e})$, telle que

$$
\rho \leq \frac{c_{17}}{C_{0}} \mathscr{H}\left(\mathbf{G} ; D_{0}^{\prime}, \ldots, D_{n}^{\prime}\right) \text { et } \nu \geq c_{17}^{-1} D \mathscr{H}\left(\mathbf{G} ; D_{0}^{\prime}, \ldots, D_{n}^{\prime}\right)
$$

où $D_{i}^{\prime}:=\max \left\{1, D_{i}\right\}$.

Démonstration. C'est le lemme 4.20 de [19], et aussi la scolie 6.13 de [25]. Le choix de $S_{0}\left(\right.$ resp. $\left.T_{0}\right)$ joue un rôle important dans cette preuve pour le cas non-périodique (resp. périodique).

Corollaire 6.19. Les entiers $D_{i}$ sont tous non nuls, i.e. $D_{i}^{\prime}=D_{i}$.

Démonstration. Supposons que, pour un indice $i \in\{0, \ldots, n\}$, l'entier $D_{i}$ soit nul. D'après le lemme 6.18, le nombre d'inconnues $\nu$ du système (31) est strictement supérieur au rang de ce système. Donc il existe un polynôme $P$, non identiquement nul sur $\mathbf{G}$, multihomogène de multidegré $\underline{D}$, solution de (31). En particulier $P$ s'annule à l'ordre $T_{0}$ en les points $s \mathbf{u}\left(0 \leq s \leq S_{0}-1\right)$ le long de $W$. Par ailleurs, comme $t_{\mathbf{G}_{i}} \not \subset W$, considérons $x \in t_{\mathbf{G}_{i}} \backslash W$. L'indépendance de la fonction $F$ vis-àvis des variables relatives à $t_{\mathbf{G}_{i}}$ (c'est l'hypothèse $D_{i}=0$ ) implique que $F$ s'annule à un ordre infini le long de la droite engendrée par $x$. Ainsi $F$ s'annule à l'ordre $T_{0}$ en les points $s \mathbf{u}\left(0 \leq s \leq S_{0}-1\right)$ le long de $(W \otimes \mathbb{C}) \oplus \mathbb{C} \cdot x=t_{\mathbf{G}}(\mathbb{C})$. D'après le lemme de zéros de P. PHiLippon [23], il existe un sous-groupe connexe et propre $\mathbf{G}^{\prime}$ de $\mathbf{G}$ tel que

$$
\begin{aligned}
T_{0}^{r^{\prime}} & \mathscr{H}\left(\mathbf{G}^{\prime} ; D_{0}^{\prime}, \ldots, D_{n}^{\prime}\right) \operatorname{card}\left(\frac{\Gamma_{\mathbf{p}}\left(\left[\frac{S_{0}-1}{d+1}\right]\right)+\mathbf{G}^{\prime}(\mathbf{L})}{\mathbf{G}^{\prime}(\mathbf{L})}\right) \\
& \leq c_{18} \mathscr{H}\left(\mathbf{G} ; D_{0}^{\prime}, \ldots, D_{n}^{\prime}\right) .
\end{aligned}
$$

Dans le cas général, nous en déduisons

$$
T_{0}^{r^{\prime}} \mathscr{H}\left(\mathbf{G}^{\prime} ; D_{0}^{\prime}, \ldots, D_{n}^{\prime}\right) \leq c_{18} \mathscr{H}\left(\mathbf{G} ; D_{0}^{\prime}, \ldots, D_{n}^{\prime}\right),
$$

(l'entier $r^{\prime}=\operatorname{codim}_{\mathbf{G}} \mathbf{G}^{\prime}$ est non nul car $\mathbf{G}^{\prime} \subsetneq \mathbf{G}$ ) ce qui contredit l'inégalité

$$
T_{0} \geq C_{0} \max \left\{1, D_{0}, \ldots, D_{n}\right\} .
$$

Dans le cas particulier où $u_{0} \neq 0$ et $\prod_{j=1}^{n} \mathbf{G}_{j}$ est une variété semi-abélienne, nous pouvons être plus précis. En effet, dans ce cas, le sous-groupe $\mathbf{G}^{\prime}$ s'écrit $\mathbf{G}_{0}^{\prime} \times \mathbf{G}^{\prime \prime}$ où $\mathbf{G}_{0}^{\prime}=\{0\}$ ou $\mathbb{G}_{\mathrm{a}}$, et où $\mathbf{G}^{\prime \prime}$ est un sous-groupe algébrique (connexe) de $\prod_{j=1}^{n} \mathbf{G}_{j}$. Si $\mathbf{G}_{0}^{\prime}=\{0\}$ alors, comme $u_{0} \neq 0$, on a nécessairement

$$
\operatorname{card}\left(\frac{\Gamma_{\mathbf{p}}\left(\left[\frac{S_{0}-1}{d+1}\right]\right)+\mathbf{G}^{\prime}(\mathbf{L})}{\mathbf{G}^{\prime}(\mathbf{L})}\right)=\left[\frac{S_{0}-1}{d+1}\right]+1
$$

et $\mathscr{H}\left(\mathbf{G}^{\prime} ; D_{0}^{\prime}, \ldots, D_{n}^{\prime}\right)=\mathscr{H}\left(\mathbf{G}^{\prime \prime} ; D_{1}^{\prime}, \ldots, D_{n}^{\prime}\right)$. L'inégalité (32) devient alors

$$
T_{0}^{r^{\prime}}\left(\frac{S_{0}-1}{d+1}\right) \leq c_{19} D_{0}^{\prime} \max \left\{D_{1}^{\prime}, \ldots, D_{n}^{\prime}\right\}^{r^{\prime}-1}
$$

ce qui contredit

$$
T_{0} \geq C_{0} \max \left\{1, \frac{D_{0}}{S_{0}}, D_{1}, \ldots, D_{n}\right\}
$$

pour $C_{0}$ assez grand.

Si $\mathbf{G}_{0}^{\prime}=\mathbb{G}_{\mathrm{a}}$ alors

$$
\mathscr{H}\left(\mathbf{G}^{\prime} ; D_{0}^{\prime}, \ldots, D_{n}^{\prime}\right)=D_{0}^{\prime} \mathscr{H}\left(\mathbf{G}^{\prime \prime} ; D_{1}^{\prime}, \ldots, D_{n}^{\prime}\right)
$$


et $\operatorname{dim} \mathbf{G}^{\prime \prime}=\operatorname{dim} \mathbf{G}^{\prime}-1=d-r^{\prime}$ donc l'inégalité (32) implique

$$
T_{0}^{r^{\prime}} \mathscr{H}\left(\mathbf{G}^{\prime \prime} ; D_{1}^{\prime}, \ldots, D_{n}^{\prime}\right) \leq c_{20} \mathscr{H}\left(\prod_{j=1}^{n} \mathbf{G}_{j} ; D_{1}^{\prime}, \ldots, D_{n}^{\prime}\right),
$$

ce qui contredit $T_{0} \geq C_{0} \max \left\{1, D_{1}, \ldots, D_{n}\right\}$.

Remarque 6.20. La non-nullité des entiers $D_{i}$ sera utilisée implicitement au début de la démonstration du lemme 6.31 (p. 30). Cependant, P. Philippon et M. WALDSCHMIDT ont montré dans 25] qu'il n'était pas absolument nécessaire de travailler avec des paramètres $D_{i}$ non nuls (un seul doit être non nul pour que les estimations du rang $\rho$ (lemme 6.18) soient non triviales). Ce n'est véritablement que dans le cas où $W$ est de codimension $>1$ (ii approximations simultanées i.ं) que leur remarque prend toute son importance, puisque, même sous des hypothèses ii raisonnables $i . i$ (du type de celles du $\S 6.1$ ), il n'est pas assuré que tous les $D_{i}$ soient non nuls. Cependant, même dans ce cas de figure, la démonstration peut s'adapter et permettre de conclure (voir [2, 18] pour une mise en situation ii concrète i.i de cette remarque).

6.9. Construction de la fonction auxiliaire. Nous utiliserons le ii classique $i \dot{i}$ :

Lemme 6.21 (de Thue-Siegel). Soit $\left(u_{i, j}\right)_{\substack{1 \leq i \leq \nu \\ 1 \leq j \leq \mu}} \in \mathrm{M}_{\nu, \mu}(\mathbb{C})$, de rang $\leq \rho$. Soient $\delta, m, p$ des réels positifs vérifiant

$$
\left(2 \mu e^{\delta+m+p}+1\right)^{2 \rho} \leq e^{\nu \delta} \text { et } \max _{1 \leq j \leq \mu}\left\{\sum_{i=1}^{\nu}\left|u_{i, j}\right|\right\} \leq e^{m} .
$$

Alors, il existe $\left(a_{1}, \ldots, a_{\nu}\right) \in \mathbb{Z}^{\nu} \backslash\{0\}$ tel que

$$
\max _{1 \leq i \leq \nu}\left\{\left|a_{i}\right|\right\} \leq e^{\delta} \text { et } \max _{1 \leq j \leq \mu}\left\{\left|\sum_{i=1}^{\nu} u_{i, j} a_{i}\right|\right\} \leq e^{-p} .
$$

La démonstration de ce résultat est effectuée dans [25] (pp. $301-303$ ). Les notations $\nu$ et $\mu$ (a priori ii muettes i.¿) ont été choisies de façon cohérente avec celles des paragraphes 6.2 et 6.8 .

Le lemme technique qui suit - majoration d'un coefficient de Taylor — est une conséquence des formules de Cauchy. Le terme ii remarquable i.i du majorant de ce coefficient de Taylor est $\varsigma_{0}^{\min \left(D_{0},|\underline{t}|\right)}$; l'exposant $\min \left(D_{0},|\underline{t}|\right)$ provient simplement du fait que la dérivée $|\underline{t}|^{\text {ème }}$ d'un monôme de degré $D_{0}$ est nulle si $|\underline{t}|>D_{0}$.

Lemme 6.22. Soient $H$ un nombre réel $\geq e, D_{0}, \ldots, D_{n} \in \mathbb{N}$ et $\ell \in \mathbb{N}^{*}$. Soit $\mathbf{x}:=\left(\mathbf{x}_{i}\right)_{i \in\{1, \ldots, \ell\}} \in\left(t_{\mathbf{G}}(\mathbb{C})\right)^{\ell} ;$ les coordonnées de $\mathbf{x}_{i}$ sont $\left(x_{i, j}\right)_{j \in\{0, \ldots, d\}}$ dans la base e de $t_{\mathbf{G}}(\mathbb{C})$. Soient $\underline{t} \in \mathbb{N}^{\ell}$ et $\mathbf{z}=z_{0} e_{0}+\cdots+z_{d} e_{d} \in t_{\mathbf{G}}(\mathbb{C})$. Soit $P$ un polynôme multihomogène de multidegré $\left(D_{0}, \ldots, D_{n}\right)$ dont la somme des modules des coefficients est $\leq H$. Rappelons que $F$ désigne la fonction $P \circ \Phi$. Considérons $\varsigma_{0}, \varsigma_{1}$ vérifiant :

$$
\varsigma_{0} \geq \max \left\{1,\left|x_{1,0}\right|, \ldots,\left|x_{\ell, 0}\right|\right\} \quad \text { et } \varsigma_{1} \geq \max _{\substack{1 \leq i \leq \ell \\ 1 \leq j \leq d}}\left\{1,\left|x_{i, j}\right|\right\}
$$

Il existe une constante $c_{21}$, ne dépendant que de $(\mathbf{G}, \Phi, \mathbf{e})$, telle que :

$$
\begin{aligned}
\left|\underline{\frac{1}{t} \underline{\mathcal{D}}} \mathcal{D} \mathbf{x} F(\mathbf{z})\right| \leq & \varsigma_{0}^{\min \left\{D_{0},|\underline{t}|\right\}}\left(\varsigma_{1} \ell(d+1)\right)^{|\underline{t}|} H\left(1+\left|z_{0}\right|\right)^{D_{0}} \\
& \times \exp \left\{c_{21} \sum_{j=1}^{n} D_{j}\left(1+\left\|p_{j}^{\prime}(\mathbf{z})\right\|\right)^{\rho_{j}}\right\}
\end{aligned}
$$

où $p_{j}^{\prime}$ désigne la projection $t_{\mathbf{G}}(\mathbb{C}) \rightarrow t_{\mathbf{G}_{j}}(\mathbb{C})$. 
Démonstration. On développe :

$$
\begin{aligned}
& \underline{\underline{t} !} \mathcal{D} \frac{t}{\mathbf{x}} F(\mathbf{z}) \\
& =\prod_{i=1}^{\ell} \frac{\left(\sum_{j=0}^{d} x_{i, j} \frac{\partial}{\partial z_{j}}\right)^{t_{i}}}{t_{i} !} F(\mathbf{z}) \\
& =\sum_{\substack{t_{i, 0}+\cdots+t_{i, d}=t_{i} \\
1 \leq i \leq \ell}}\left(\prod_{i, j} \frac{\left(x_{i, j}^{t_{i, j}}\right)}{\left(t_{i, j}\right) !}\right) \prod_{h=0}^{d}\left(\frac{\partial}{\partial z_{h}}\right)^{t_{1, h}+\cdots+t_{\ell, h}} F(\mathbf{z}) \\
& =\sum_{t_{i, 0}+\cdots+t_{i, d}=t_{i}}\left\{\left(\prod_{i, j} x_{i, j}^{t_{i, j}}\right) \times\left(\prod_{h=0}^{d} \frac{\left(t_{1, h}+\cdots+t_{\ell, h}\right) !}{t_{1, h} ! \cdots t_{\ell, h} !}\right) \times \prod_{h=0}^{d} \frac{\left(\frac{\partial}{\partial z_{h}}\right)^{t_{1, h}+\cdots+t_{\ell, h}}}{\left(t_{1, h}+\cdots+t_{\ell, h}\right) !} F(\mathbf{z})\right\} .
\end{aligned}
$$

Dans le membre de droite ci-dessus, nous pouvons restreindre la somme à $t_{1,0}+$ $\cdots+t_{\ell, 0} \leq D_{0}$ car $F$ est polynomiale en $z_{0}$ de degré au plus $D_{0}$. Nous en déduisons la majoration :

$$
\begin{aligned}
& \left.\left|\frac{1}{\underline{t} !} \mathcal{D} \frac{t}{\mathbf{x}} F(\mathbf{z})\right| \leq \varsigma_{0}^{\min \left(D_{0},|\underline{t}|\right)} \times \varsigma_{1}^{\mid \underline{|t|} \times(} \sum_{\substack{t_{i, 0}+\cdots+t_{i, d}=t_{i} \\
1 \leq i \leq \ell}} \prod_{h=0}^{d} \frac{\left(t_{1, h}+\cdots+t_{\ell, h}\right) !}{t_{1, h} ! \cdots t_{\ell, h} !}\right) \\
& \times \max _{\mid \underline{\underline{\tau}|=| \underline{t} \mid}}\left\{\left|\frac{1}{\tau !} \mathcal{D} \mathcal{D} \frac{\tau}{\mathbf{e}} F(\mathbf{z})\right|\right\} .
\end{aligned}
$$

Comme $\prod_{h=0}^{d}\left(t_{1, h}+\cdots+t_{\ell, h}\right) ! \leq\left(\sum_{i, j} t_{i, j}\right) !=(|\underline{t}|) !$, on a

$$
\sum_{\substack{t_{i, 0}+\cdots+t_{i, d}=t_{i} \\ 1 \leq i \leq \ell}} \prod_{h=0}^{d} \frac{\left(t_{1, h}+\cdots+t_{\ell, h}\right) !}{t_{1, h} ! \cdots t_{\ell, h} !} \leq\{\ell(d+1)\}^{|\underline{t}|}
$$

et donc

$$
\left|\frac{1}{\underline{t} !} \mathcal{D} \frac{\underline{t}}{\mathbf{x}} F(\mathbf{z})\right| \leq \varsigma_{0}^{\min \left(D_{0},|\underline{t}|\right)} \times \varsigma_{1}^{|\underline{t}|} \times\{\ell(d+1)\}^{|\underline{t}|} \times \max _{\mid \underline{\underline{I}|=| \underline{t} \mid}}\left\{\left|\frac{1}{\tau \underline{\underline{\tau}}} \mathcal{D} \mathcal{D}_{\mathbf{e}}^{\underline{\tau}} F(\mathbf{z})\right|\right\} .
$$

D'après l'inégalité de Cauchy, si nous posons

$$
\mathbf{z}_{\theta}:=e^{i \theta_{0}} e_{0}+\cdots+e^{i \theta_{d}} e_{d}
$$

on a

$$
\begin{aligned}
\left|\frac{1}{\underline{\tau} !} \mathcal{D} \frac{\tau}{\mathbf{e}} F(\mathbf{z})\right| \leq & \sup _{\theta_{j} \in[0,2 \pi]}\left\{\left|F\left(\mathbf{z}+\mathbf{z}_{\theta}\right)\right|\right\} \\
\leq & H \times\left(1+\left|z_{0}\right|\right)^{D_{0}} \\
& \times \sup _{\theta \in[0,2 \pi]^{d+1}}\left\{\prod_{j=1}^{n} \exp \left\{c_{j}^{+} D_{j}\left(1+\left\|p_{j}^{\prime}\left(\mathbf{z}+\mathbf{z}_{\theta}\right)\right\|_{j}\right)^{\rho_{j}}\right\}\right\}
\end{aligned}
$$

(cf. inégalité (13), p. 13).

Alors, comme $1+\left\|p_{j}\left(\mathbf{z}+\mathbf{z}_{\theta}\right)\right\|_{j} \leq 1+\| p_{j}\left(\mathbf{z} \|_{j}+\sqrt{\delta_{j}}\right.$, nous obtenons

$$
\left|\frac{1}{\underline{\tau} !} \mathcal{D} \frac{\tau}{\mathbf{e}} F(\mathbf{z})\right| \leq H\left(1+\left|z_{0}\right|\right)^{D_{0}} \prod_{j=1}^{n} \exp \left\{c_{j}^{+} D_{j}\left(1+\left\|p_{j}(\mathbf{z})\right\|_{j}+\sqrt{\delta_{j}}\right)^{\rho_{j}}\right\} .
$$

Ce qui, via l'inégalité (34), donne le résultat voulu.

Remarque 6.23. Au vu de la démonstration, il apparaît clairement que l'on peut améliorer substantiellement cette majoration en considérant le vecteur $r_{0} e^{i \theta_{0}} w_{0}+$ $\cdots+r_{d} e^{i \theta_{d}} w_{d}$ au lieu de $\mathbf{z}_{\theta}=e^{i \theta_{0}} w_{0}+\cdots+e^{i \theta_{d}} w_{d}$ et en ajustant $\left(r_{0}, \ldots, r_{d}\right)$ pour minimiser le membre de droite de (35). De cette manière, il est possible de faire apparaître (dans le majorant de $\left.\frac{1}{\underline{t} !}\left|\mathcal{D} \frac{t}{\mathbf{x}} F(\mathbf{z})\right|\right)$ un facteur de la forme

$$
\min \left(1, \frac{D_{0}+\cdots+D_{n}}{|\underline{t}|}\right)^{|\underline{t}| / 2} \text {. }
$$


Mais, outre les complications inhérentes à cette optimisation, il est décevant de constater que, dans le cadre de notre étude, ce raffinement est inexploitable et dénué d'intérêt (sauf si on cherche à optimiser la constante dépendant de $\mathbf{G}$ ), car le terme ainsi amélioré sera ii négligeable $i: i$ devant d'autres termes.

Proposition 6.24. Pour $C_{0}$ assez grand, il existe un polynôme multihomogène $P$ de $\mathbf{L}[\mathbb{P}]$, de multidegré $\left(D_{0}, \ldots, D_{n}\right)$, ne s'annulant pas identiquement sur $\mathbf{G}$, tel que :

- Les coefficients de $P$ appartiennent à $\mathbb{Z} . \xi_{1} \oplus \cdots \oplus \mathbb{Z} . \xi_{D}$.

- La hauteur $h(P)$ du polynôme P est majorée par :

$$
C_{0}^{1 / 2} \frac{U_{0}}{D}
$$

- En posant $F=P \circ \Phi$ comme précédemment :

$$
\max _{(\underline{t}, s) \in \Upsilon}\left\{\left|\frac{1}{\underline{t} !} \mathcal{D} \frac{\underline{t}}{\mathbf{f}} F(s \mathbf{u})\right|\right\} \leq e^{-C_{0} U_{0}} .
$$

Démonstration. On écrit, a priori, $P=\sum_{\underline{\lambda}, i} a_{\underline{\lambda}}, i \xi_{i} \mathbf{X} \underline{\underline{\lambda}}$ où $a_{\underline{\lambda}, i} \in \mathbb{Z}$ et $\mathbf{X} \underline{\underline{\lambda}} \in \mathcal{B}$ (cf. $\S$ 6.8). Pour $(\underline{t}, s) \in \Upsilon$, posons

$$
u_{(\underline{\lambda}, i)(\underline{t}, s)}=\xi_{i} \frac{1}{\underline{t} !} \mathcal{D}_{\underline{\mathbf{f}}}^{\underline{\mathbf{f}}}\left(\Phi^{\underline{\lambda}}\right)(s \mathbf{u}) .
$$

On notera $p_{\underline{\lambda}}:=\sum_{i} a_{\underline{\lambda}, i} \xi_{i}$ et $\mu$ le cardinal de $\Upsilon$. La matrice

$$
\left(u_{(\underline{\lambda}, i)(\underline{t}, s)}\right)_{\substack{(\underline{\lambda}, i) \\, s)}} \in \mathrm{M}_{\nu, \mu}(\mathbb{C})
$$

est de rang $\rho$ (par définition de $\rho$ (lemme 6.18)). Considérons

$$
\varsigma_{0}=(d+1)^{1 / 2} \max \left\{1,\left|\beta_{1}\right|, \ldots,\left|\beta_{d}\right|\right\}=(d+1)^{1 / 2}
$$

$\left(\operatorname{car}\left|\beta_{i}\right| \leq 1\right.$, voir p. 14) et

$$
\varsigma_{1}= \begin{cases}1 & \text { dans le cas non-périodique, } \\ 1+\|\mathbf{u}\| & \text { dans le cas périodique. }\end{cases}
$$

Appliquons le lemme 6.22 au monôme $\mathbf{X} \underline{\lambda}$, à la base $\mathbf{x}:=\mathbf{f}$, et au vecteur $\mathbf{z}:=s \mathbf{u}$ :

$$
\begin{aligned}
\left|\underline{\underline{t} !} \mathcal{L} \frac{\underline{t}}{\mathbf{f}}\left(\Phi^{\underline{\lambda}}\right)(s \mathbf{u})\right| \leq & (d+1)^{\min \left\{D_{0},|\underline{t}|\right\} / 2} \times \varsigma_{1}^{|\underline{\mid t}|} \times\{(d+1)(d+2)\}^{|\underline{t}|} \\
& \times\left(1+s\left\|\mathbf{u}_{0}\right\|\right)^{D_{0}} \times \exp \left\{c_{22} \sum_{j=1}^{n} D_{j}\left(1+s\left\|\mathbf{u}_{j}\right\|\right)^{\rho_{j}}\right\} \\
\leq & c_{23}^{T} \times\left(1+S\left\|\mathbf{u}_{0}\right\|\right)^{D_{0}} \times e^{c_{23} \sum_{j=1}^{n} D_{j}\left(1+S\left\|\mathbf{u}_{j}\right\|\right)^{\rho_{j}}} \\
\leq & e^{c_{24} U_{0}} .
\end{aligned}
$$

Ainsi, il existe une constante $c_{25}$, ne dépendant que de $(\mathbf{G}, \Phi, \mathbf{e})$, telle que :

$$
\begin{aligned}
\max _{(\underline{\underline{t}}, s) \in \Upsilon}\left\{\sum_{\underline{\lambda}}\left|\frac{1}{\underline{\underline{t}}} \mathcal{D}^{\underline{\underline{t}}}\left(\Phi^{\underline{\lambda}}\right)(s \mathbf{u})\right|\right\} & \leq \mathscr{H}\left(\mathbf{G} ; D_{0}, \ldots, D_{n}\right) e^{c_{25} U_{0}} \\
& \leq e^{2 c_{25} U_{0}}
\end{aligned}
$$

car $\mathscr{H}\left(\mathbf{G} ; D_{0}, \ldots, D_{n}\right) \leq\left(\operatorname{deg}_{\Phi} \mathbf{G}\right) U_{0}^{n+1} \leq e^{c_{25} U_{0}}$, pour $C_{0}$ assez grand. Comme

$$
\begin{aligned}
\sum_{i=1}^{D}\left|\xi_{i}\right| & \leq D \max \left\{\left|\xi_{1}\right|, \ldots,\left|\xi_{D}\right|\right\} \\
& \leq D \times e^{D h\left(\xi_{1}: \cdots: \xi_{D}\right)} \\
& \leq e^{U_{0}}
\end{aligned}
$$


nous en déduisons une majoration de :

$$
\begin{aligned}
\max _{(\underline{t}, s) \in \Upsilon}\left\{\sum_{(\underline{\lambda}, i)}\left|u_{(\underline{\lambda}, i),(\underline{t}, s)}\right|\right\} & \leq\left(\sum_{i=1}^{D}\left|\xi_{i}\right|\right) \times \max _{(\underline{t}, s) \in \Upsilon}\left\{\sum_{\underline{\lambda}}\left|\frac{1}{\underline{t} !} \mathcal{D}^{\underline{t}}\left(\Phi^{\underline{\lambda}}\right)(s \mathbf{u})\right|\right\} \\
& \leq e^{c_{26} U_{0}} .
\end{aligned}
$$

Par ailleurs, $\nu \geq c_{27} D \mathscr{H}\left(\mathbf{G} ; D_{0}, \ldots, D_{n}\right)$ et

(lemme 6.18 et corollaire 6.19) donc

$$
\frac{1}{\rho} \geq \frac{C_{0}}{c_{28}} \cdot \frac{1}{\mathscr{H}\left(\mathbf{G} ; D_{0}, \ldots, D_{n}\right)}
$$

$$
\frac{\nu}{2 \rho} \geq \frac{C_{0} D}{c_{29}} .
$$

Pour que la condition (du lemme de Thue-Siegel)

$$
\left(2 \mu e^{\delta+m+p}+1\right)^{2 \rho} \leq e^{\nu \delta}
$$

soit vérifiée, nous choisissons $p:=C_{0} U_{0}, m:=c_{30} U_{0}$ et $\delta:=C_{0}^{1 / 4} \frac{U_{0}}{D}$ (voir également la majoration de $\mu$ p. 18). Ainsi, le lemme de Thue-Siegel permet de construire un tel polynôme $P$, de coefficients $p_{\underline{\lambda}}=\sum_{i} a_{\underline{\lambda}, i} \xi_{i}$, avec $a_{\underline{\lambda}, i} \in \mathbb{Z}$ non tous nuls et $\max _{\underline{\lambda}, i}\left\{\left|a_{\underline{\lambda}, i}\right|\right\} \leq e^{\delta}$. Par conséquent :

- Pour une place $\mathfrak{P}$ ultramétrique :

$$
\left|p_{\underline{\lambda}}\right|_{\mathfrak{P}} \leq \max _{1 \leq i \leq D}\left\{\left|\xi_{i}\right|_{\mathfrak{P}}\right\} .
$$

- Pour une place $\sigma$ archimédienne :

$$
\begin{aligned}
\left|p_{\underline{\lambda}}\right|_{\sigma} & \leq D \max _{i}\left\{\left|a_{\underline{\lambda}, i}\right|\right\} \max _{1 \leq i \leq D}\left\{\left|\xi_{i}\right|_{\sigma}\right\} \\
& \leq\left(D e^{\delta}\right) \max _{1 \leq i \leq D}\left\{\left|\xi_{i}\right|_{\sigma}\right\} .
\end{aligned}
$$

Donc

$$
h(P) \leq \log \left(D e^{\delta}\right)+h\left(\xi_{1}: \cdots: \xi_{D}\right) \leq \log D+C_{0}^{1 / 4} \frac{U_{0}}{D}+\frac{U_{0}}{D} \leq C_{0}^{1 / 2} \frac{U_{0}}{D}
$$

pour $C_{0}$ assez grand. Ce qui clôt la démonstration.

Tout le travail préparatoire (élimination des éventuels ii mauvais i¿ sous-groupes et construction du polynôme auxiliaire) étant effectué, le schéma de démonstration est le suivant : considérons $\frac{1}{\underline{t} !} \mathcal{D} \frac{t}{\widetilde{\mathbf{e}}} F(m \mathbf{u})$ le premier ${ }^{8}$ coefficient de Taylor non nul. Un tel coefficient existe car, dans le cas contraire, le lemme de zéros de Philippon entrerait en conflit avec le choix des paramètres (voir lemme 6.31, p. 30). Ce terme est proportionnel à un élément $\Xi \in \mathbf{L}$.

(1) Nous évaluons la norme $\mathfrak{P}$-adique de $\Xi(\S 6.11)$.

(2) Nous évaluons la norme $\sigma$-archimédienne de $\Xi$, pour tout plongement $\sigma$ : $\mathbf{L} \rightarrow \mathbb{C}(\S 6.12)$.

(3) La quantité $\frac{1}{j !} \mathcal{D} \frac{j}{\mathbf{f}} F(s \mathbf{u})$ est ii petite $i . i$ pour $(\underline{j}, s) \in \Upsilon$ (et relativement à la valeur abssolue associée au plongement initial $\sigma_{0}$ de $\mathbf{L}$ dans $\mathbb{C}$ ). Nous commençons alors un raisonnement par l'absurde et nous supposons que la distance de $\mathbf{u}$ à $W$ est non nulle et plus petite qu'une certaine quantité. Par extrapolation, nous montrons alors que le terme $\frac{1}{\underline{j} !} \mathcal{D} \frac{j}{\mathbf{f}} F(s \mathbf{u})$ reste ii petit i.i pour $|\underline{j}| \leq(d+1) T$ et $s \leq(d+1) S$. Nous en déduisons alors, par changement de base, une estimation de $\frac{1}{\underline{t} !} \mathcal{D} \underset{\widetilde{\mathbf{e}}}{\underline{t}} F(m \mathbf{u})$ puis de $\Xi$ (c'est l'objet $\mathrm{du}$ paragraphe suivant).

\footnotetext{
${ }^{8}$ Pour l'ordre lexicographique sur $\mathbb{N} \times \mathbb{N}^{d}$.
} 
(4) Nous aurons alors un nombre algébrique $\Xi$ ii petit ¿i. pour toutes les valeurs absolues associées aux différentes places de $\mathbf{L}$. La formule du produit impliquera la nullité de ce nombre. Ce qui est en contradiction avec la définition du couple $(\underline{t}, m)$. Nous en déduirons alors la minoration idoine de $|\Lambda|$.

6.10. Extrapolation. À partir de maintenant nous commençons un raisonnement par l'absurde et nous supposons que

$$
|\Lambda| \leq e^{-C_{0} U_{0}}
$$

Voici un lemme préliminaire :

Lemme 6.25. Soit $F$ la fonction holomorphe associée au polynôme construit précédemment. Il existe une constante $c_{31}>0$, ne dépendant que de $(\mathbf{G}, \Phi, \mathbf{e})$, telle que, pour tout couple $(\underline{t}, s) \in \mathbb{N}^{d+1}$ avec $|\underline{t}| \leq 2(d+1) T$ et $s \leq 2(d+1) S$, l'inégalité suivante soit vérifiée :

$$
\left|\frac{1}{\underline{t} !} \mathcal{D} \underline{\underline{f}} F(s \mathbf{u})-\frac{1}{t} \mathcal{L} \mathcal{D}_{\mathbf{f}}^{\underline{t}} F(s \mathbf{w})\right| \leq e^{-c_{31} C_{0} U_{0}} .
$$

Démonstration. C'est une conséquence de l'inégalité des accroissements finis. Considérons, pour un couple $(\underline{t}, s)$ et un réel $x$, la fonction

$$
f(x)=\frac{1}{\underline{t} !} \mathcal{D} \frac{t}{\mathbf{f}} F(s \mathbf{u}+x s(\mathbf{w}-\mathbf{u})) .
$$

Cette fonction est dérivable sur $[0,1]$ et donc $|f(0)-f(1)| \leq \max _{x \in[0,1]}\left|f^{\prime}(x)\right|$. Or

$$
f^{\prime}(x)=\sum_{i=0}^{d} s\left(w_{i}-u_{i}\right) \frac{\partial}{\partial z_{i}}\left(\frac{1}{\underline{t} !} \mathcal{D} \frac{t}{\mathbf{f}} F(s \mathbf{u}+x s(\mathbf{w}-\mathbf{u}))\right)
$$

donc

$$
\left|f^{\prime}(x)\right|=s|\Lambda|\left|\frac{\partial}{\partial z_{0}}\left(\frac{1}{\underline{t} !} \mathcal{D} \frac{t}{\mathbf{f}} F(s \mathbf{u}+x s(\mathbf{w}-\mathbf{u}))\right)\right| .
$$

La majoration (39) découle du lemme 6.22 appliqué à $\ell:=d+1, \mathbf{x}:=\left(e_{0}, \mathbf{f}\right)$, $\underline{t}^{\prime}:=(1, \underline{t}), \mathbf{z}:=s \mathbf{u}+x s(\mathbf{w}-\mathbf{u}), H:=e^{C_{0}^{1 / 2} U_{0}}, \varsigma_{0}:=(d+1)^{1 / 2}$ et $\varsigma_{1}:=1+\|\mathbf{u}\|$ (on a pris 1 dans le cas non-périodique), et de l'inégalité $2(d+1) S|\Lambda| \leq 1$ (conséquence de l'hypothèse (38)).

La proposition suivante est le cœur de l'extrapolation (analytique) que nous voulons effectuer. Sa preuve repose sur un lemme de Schwarz approché.

Proposition 6.26. Pour $C_{0}$ assez grand, pour tout réel $E \geq 1$, pour tout $(\underline{t}, s) \in$ $\mathbb{N}^{d+1}$ avec $|\underline{t}| \leq(d+1) T$ et $s \leq(d+1) S$, on a l'inégalité

$$
\left|\frac{1}{\underline{t} !} \mathcal{D} \frac{\underline{t}}{\mathbf{f}} F(s \mathbf{u})\right| \leq \exp \left\{-C_{0}^{3 / 4} U_{0}\right\} .
$$

Démonstration. Par construction de $P$, pour tout $(\underline{t}, s) \in \Upsilon$, on a

$$
\begin{aligned}
\left|\frac{1}{\underline{t} !} \mathcal{D} \frac{t}{\mathbf{f}} F(s \mathbf{w})\right| & \leq\left|\frac{1}{\underline{t} !} \mathcal{D} \frac{\underline{t}}{\mathbf{f}} F(s \mathbf{u})\right|+\left|\frac{1}{\underline{t} !} \mathcal{D} \frac{\underline{t}}{\mathbf{f}} F(s \mathbf{u})-\frac{1}{\underline{t} !} \mathcal{D} \frac{t}{\mathbf{f}} F(s \mathbf{w})\right| \\
& \leq e^{-c_{32} C_{0} U_{0}}
\end{aligned}
$$

pour une certaine constante $c_{32}$, ne dépendant que de $(\mathbf{G}, \Phi, \mathbf{e})$.

- Dans le cas non-périodique, fixons un $d$-uplet $\underline{t}=\left(t_{1}, \ldots, t_{d}\right)$ tel que $t_{1}+\cdots+t_{d} \leq$ $(d+1) T$ et posons

$$
f(z)=\frac{1}{\underline{t} !} \mathcal{D} \frac{t}{\mathbf{f}} F(z \mathbf{w}) .
$$

- Dans le cas périodique, nous fixons également $\underline{t}=\left(t_{1}, \ldots, t_{d-1}, t_{d}\right)$ avec $|\underline{t}| \leq$ $(d+1) T$ mais nous posons

$$
f(z)=\frac{1}{t_{1} ! \cdots t_{d-1} !} \mathcal{D}_{\mathbf{f}}^{\left(t_{1}, \ldots, t_{d-1}, 0\right)} F(z \mathbf{w}) .
$$


La fonction complexe $f$ ainsi définie est analytique, et les dérivées sont

$$
\frac{1}{m !} f^{(m)}(z)=\sum_{\substack{\underline{j} \in \mathbb{N}^{d} \\
j_{1}+\cdots+j_{d}=m}}\left(\begin{array}{c}
\underline{t}+\underline{j} \\
\underline{j}
\end{array}\right) \mathfrak{w}_{1}^{j_{1}} \cdots \mathfrak{w}_{d}^{j_{d}} \frac{1}{(\underline{t}+\underline{j}) !} \mathcal{D}_{\mathbf{f}}^{\underline{t}+\underline{j}}-F(z \mathbf{w})
$$

où $\mathfrak{w}_{1}, \ldots, \mathfrak{w}_{d}$ sont les composantes de $\mathbf{w}$ dans la base $\mathbf{f}$. Le ii lemme de Schwarz approché $i$ i, évoqué en préambule de la proposition, qui est au centre du processus d'extrapolation est le suivant. Notons $|f|_{R}=\sup _{|z| \leq R}\{|f(z)|\}$.

Lemme 6.27 ([30). Soient $f$ une fonction analytique dans le disque de centre 0 et de rayon $R \geq 4, S_{1}$ un entier $\geq 2, r \in\left[S_{1}, R / 2\right]$ et $T_{1}$ un entier naturel. Alors

$$
|f|_{2 r} \leq 2|f|_{R}\left(\frac{4 r}{R}\right)^{T_{1} S_{1}}+5\left(\frac{18 r}{S_{1}}\right)^{T_{1} S_{1}} \times \max _{\substack{0 \leq m<T_{1} \\ 0 \leq h<S_{1}}}\left\{\left|\frac{1}{m !} f^{(m)}(h)\right|\right\} .
$$

(1) Cas non-périodique

Nous allons appliquer le lemme précédent, pour extrapoler sur les points, avec $r:=(d+1) S / 2, R:=2(d+1) S E, T_{1}:=(d+1) T$ et $S_{1}:=S_{0}$. L'égalité 42 permet de majorer

par

$$
\max \left\{\left|\frac{1}{m !} f^{(m)}(h)\right| ; 0 \leq m<(d+1) T \text { et } 0 \leq h<S_{0}\right\}
$$

$$
\left(d+\left|\mathfrak{w}_{1}\right|+\cdots+\left|\mathfrak{w}_{d}\right|\right)^{2(d+1) T} \times \max _{(\underline{h}, s) \in \Upsilon}\left|\frac{1}{h !} \underline{D} \frac{\underline{h}}{\mathbf{f}} F(s \mathbf{w})\right|
$$

puis, via l'inégalité (41), par $e^{-c_{33} C_{0} U_{0}}$. De plus, d'après la proposition 6.16 (changement de bases de dérivation) et comme $2(d+1) S E|\Lambda| \leq 1$ (i.e. $R|\Lambda| \leq 1$, c'est encore une conséquence de l'hypothèse $(38)$ ), on a

$$
|f|_{R} \leq d^{3(d+1) T / 2} \times \max _{\substack{|z|=R \\|\underline{h}| \leq(d+1) T}}\left\{\left|\frac{1}{\underline{h} !} \mathcal{D} \frac{\underline{h}}{\widetilde{\mathbf{e}}} F(z \mathbf{w})\right|\right\} \leq e^{c_{34} U_{0}} .
$$

Le lemme 6.27 permet alors d'obtenir la majoration annoncée du coefficient de Taylor $\left|\frac{1}{\underline{t} !} \mathcal{D} \frac{t}{\mathbf{f}} F(s \mathbf{u})\right|$.

2 Cas périodique

Dans ce cas, par définition de la base $\mathbf{f}$, qui contient le vecteur $\mathbf{w}$, la formule de dérivation (42) se simplifie :

$$
\forall m \in \mathbb{N}, \quad \frac{1}{m !} f^{(m)}(z)=\frac{1}{t_{1} ! \cdots t_{d-1} ! m !} \mathcal{D}_{\mathbf{f}}^{\left(t_{1}, \ldots, t_{d-1}, m\right)} F(z \mathbf{w}) .
$$

Par conséquent, la construction de $P$ et le lemme 6.25 impliquent que le terme

$$
\max _{\substack{0 \leq m \leq T_{0} \\ 0 \leq s<2(d+1) S}}\left\{\left|\frac{1}{m !} f^{(m)}(s)\right|\right\}
$$

est inférieur à $e^{-c_{35} C_{0} U_{0}}$. Nous appliquons le lemme d'interpolation avec $R:=4(d+$ 1) $S E, T_{1}:=T_{0}, r=S_{1}:=(d+1) S$. L'inégalité de Cauchy :

$$
\left|\frac{1}{t_{d} !} f^{\left(t_{d}\right)}(s)\right| \leq|f|_{(d+1) S+1} \leq|f|_{2(d+1) S},
$$

pour $s \in\{0, \ldots,(d+1) S\}$, et, de nouveau, le lemme 6.27 permettent d'obtenir la majoration voulue.

Corollaire 6.28. Pour $C_{0}$ assez grand, pour tout $(\underline{t}, s) \in \mathbb{N}^{d+1}$ avec $|\underline{t}| \leq(d+1) T$ et $s \leq(d+1) S$, la quantité $\left|\frac{1}{\underline{t} !} \mathcal{D} \frac{t}{\widetilde{\mathbf{e}}} F(s \mathbf{u})\right|$ est majorée par $\exp \left\{-C_{0}^{5 / 8} U_{0}\right\}$. 
Démonstration. Nous appliquons la proposition 6.16 de changement de bases de dérivations. Dans le cas non-périodique, le corollaire est immédiat. Dans le cas périodique, avec la remarque qui suit la proposition 6.16 , nous avons

$$
\begin{aligned}
\log & \left|\frac{1}{\underline{t} !} \mathcal{D} \underset{\tilde{\mathbf{e}}}{\underline{t}} F(s \mathbf{u})\right| \\
\leq & c_{36} T \log \left(C_{0}^{23 d}\left(D+\sum_{i=1}^{n}\left(D h\left(\mathbf{p}_{i}\right)+E\left\|\mathbf{u}_{i}\right\|\right)\right)^{20 d}\right) \\
& +\max _{|\underline{t}| \leq(d+1) T} \log \left|\frac{1}{\underline{t} !} \mathcal{D}_{\mathbf{f}}^{\underline{t}} F(s \mathbf{u})\right| \\
\leq & C_{0}^{1 / 4} U_{0}-C_{0}^{3 / 4} U_{0} \leq-C_{0}^{5 / 8} U_{0} \quad \text { pour } C_{0} \text { assez grand }
\end{aligned}
$$

Ce corollaire conclut la partie analytique de la démonstration. Le paragraphe suivant a trait à la partie arithmétique qui est au centre de ce texte.

6.11. Estimations ultramétriques. Notons $M_{\mathbf{L}, f}$ l'ensemble des places finies de L

Définition 6.29. Soient $n$ et $k$ des entiers naturels non nuls. Nous noterons $\delta_{k}(n)$ l'entier :

$$
\operatorname{ppcm}\left\{i_{1} \cdots i_{h} ; i_{j} \in \mathbb{N}^{*}, h \leq n, i_{1}+\cdots+i_{h} \leq k\right\} .
$$

Dans [8], S. Bruiltet a démontré ${ }^{9}$ le

Lemme 6.30. Pour tout $n, k \in \mathbb{N}^{*}$, on a $\log \delta_{k}(n) \leq k \log (4 n)$.

Lemme 6.31. Parmi les nombres $\frac{1}{\underline{t !}} \mathcal{D} \frac{t}{\widetilde{\mathbf{e}}} F(m \mathbf{u})$, avec $0 \leq|\underline{t}| \leq(d+1) T$ et $0 \leq m \leq$ $(d+1) S$, au moins un n'est pas nul.

Démonstration. Si tous ces nombres complexes $\mathcal{D} \underset{\widetilde{\mathbf{e}}}{\underline{t}} F(m \mathbf{u})$ étaient nuls, alors (par définition) $P$ s'annulerait à l'ordre $(d+1) T$ le long de $W$ en tous les points $s \mathbf{u}$, $s \in\{0, \ldots,(d+1) S\}$ et, d'après le lemme de zéros de P. Philippon, il existerait un sous-groupe connexe et propre $\mathbf{G}^{\prime}$ de $\mathbf{G}$ vérifiant l'inégalité

$$
\begin{aligned}
& T^{\operatorname{codim}_{W}\left(W \cap t_{\mathbf{G}^{\prime}}\right)} \operatorname{card}\left(\frac{\Gamma_{\mathbf{p}}(S)+\mathbf{G}^{\prime}(\mathbf{L})}{\mathbf{G}^{\prime}(\mathbf{L})}\right) \mathscr{H}\left(\mathbf{G}^{\prime} ; D_{0}, \ldots, D_{n}\right) \\
& \leq c_{37} \mathscr{H}\left(\mathbf{G} ; D_{0}, \ldots, D_{n}\right) .
\end{aligned}
$$

La proposition 6.2 exclut le cas où $t_{\mathbf{G}^{\prime}} \subseteq W$. Le même argument que celui donné à la fin de la preuve du corollaire 6.19 élimine l'autre cas. Donc $\mathbf{G}^{\prime}$ ne peut pas exister et le lemme est démontré.

Fixons un couple $(m, \underline{t})$ tel que $\mathcal{D} \frac{t}{\widetilde{\mathbf{e}}} F(m \mathbf{u}) \neq 0$ et $\mathcal{D} \frac{h}{\widetilde{\mathbf{e}}} F(m \mathbf{u})=0$ pour tout $d$-uplet $\underline{h}$ de longueur $<|\underline{t}|$. Soit $\left(\tau_{0}, \ldots, \tau_{n}\right) \in\left\{0, \ldots, N_{0}\right\} \times \cdots \times\left\{0, \ldots, N_{n}\right\}$ tel que $\left|\varphi_{\tau_{j}}^{(j)}\left(m \mathbf{u}_{j}\right)\right|$ soit non-nul pour $0 \leq j \leq n$. Si nous regardons $F$ comme une section du fibré $\mathcal{O}_{\mathbf{G}}\left(D_{0}, \ldots, D_{n}\right)$, le choix de $\left(\tau_{0}, \ldots, \tau_{n}\right)$ correspond au choix d'une trivialisation de cette section au voisinage de $m \mathbf{p}$.

Lemme 6.32. Le nombre complexe

$$
\frac{1}{\prod_{j=0}^{n}\left(\varphi_{\tau_{j}}^{(j)}\left(m \mathbf{u}_{j}\right)\right)^{D_{j}}} \frac{1}{\underline{t} !} \mathcal{D} \frac{\underline{\underline{\mathbf{e}}}}{\mathrm{e}} F(m \mathbf{u})
$$

est un élément de $\mathbf{L}$.

\footnotetext{
${ }^{9}$ On dispose aussi (ibid.) de l'estimation asymptotique $\frac{1}{k} \log \delta_{k}(n) \underset{k \rightarrow+\infty}{\longrightarrow} \sum_{i=1}^{n} \frac{1}{i}$.
} 
Démonstration. En vertu de la formule de Leibniz, le nombre complexe (44) est la dérivée (divisée, le long de $W$, à l'ordre $\underline{t}$, au point $m \mathbf{u}$ ) de la fonction

$$
\frac{F(\mathbf{z})}{\prod_{j=0}^{n}\left(\varphi_{\tau_{j}}^{(j)}(\mathbf{z})\right)^{D_{j}}}
$$

qui est un polynôme en $\left(\psi_{\tau_{j}}^{(j)}\right)_{j=0, \ldots, n}$ (voir définition de $\psi_{\tau_{j}}^{(j)}$, p. 14). Les formules de dérivations liées aux groupes $\mathbf{G}_{j}(\mathrm{du}$ type de celle $(14)$, p. 13) permettent alors d'en déduire ce lemme.

Le point clef de la démonstration des théorèmes 3.1 et 3.2 est le

Lemme 6.33. Il existe un ensemble fini $\mathcal{S}$ de places de $\mathbf{L}$, dont la trace sur $\mathbf{K}$ (i.e. $\left.\left\{\mathfrak{P} \cap \mathcal{O}_{\mathbf{K}} ; \mathfrak{P} \in \mathcal{S}\right\}\right)$ ne dépend que de $(\mathbf{G}, \Phi, \mathbf{e})$, il existe des constantes $\left(\mathbf{c}_{j}\right)_{j \in\{0, \ldots, n\}}$ $\left(\mathbf{c}_{0}=1\right)$ ne dépendant que de $(\mathbf{G}, \Phi, \mathbf{e})$, tels que, pour toute place $\mathfrak{P} \notin \mathcal{S}$, l'inégalité suivante soit vérifiée :

$$
\begin{aligned}
& \left|\frac{1}{\prod_{j=0}^{n}\left(\varphi_{\tau_{j}}^{(j)}\left(m \mathbf{u}_{j}\right)\right)^{D_{j}}} \frac{1}{\underline{t} !} \mathcal{D} \underline{\underline{\underline{e}}} F(m \mathbf{u})\right|_{\mathfrak{P}} \\
& \leq \\
& \max \left\{1,\left|\beta_{1}\right|_{\mathfrak{P}}, \ldots,\left|\beta_{d}\right|_{\mathfrak{P}}\right\}^{D_{0}} \max _{\underline{\underline{\lambda}}}\left\{\left|p_{\underline{\lambda}}\right|_{\mathfrak{P}}\right\} \\
& \quad \times \frac{1}{\left|\delta_{|\underline{t}|}\left(D_{0}\right)\right|_{\mathfrak{P}}} \prod_{h=0}^{n} \frac{1}{\left|A_{\tau_{h}}^{(h)}\left(\psi_{0}^{(h)}(0), \psi_{\tau_{h}}^{(h)}\left(m \mathbf{u}_{h}\right)\right)\right|_{\mathfrak{P}}^{D_{h}}} \\
& \quad \times \prod_{j=0}^{n} \max \left\{\left|\frac{\varphi_{0}^{(j)}\left(m \mathbf{u}_{j}\right)}{\varphi_{\tau_{j}}^{(j)}\left(m \mathbf{u}_{j}\right)}\right|_{\mathfrak{P}}, \cdots,\left|\frac{\varphi_{N_{j}}^{(j)}\left(m \mathbf{u}_{j}\right)}{\varphi_{\tau_{j}}^{(j)}\left(m \mathbf{u}_{j}\right)}\right|_{\mathfrak{P}}^{\mathbf{c}_{j} D_{j}}\right.
\end{aligned}
$$

où $|\cdot|_{\mathfrak{P}}$ est la valeur absolue sur $K_{\mathfrak{P}}$ définie $p .3$.

Démonstration. Nous avons pris le parti de n'utiliser ici que des notions très élémentaires de géométrie algébrique. Une preuve plus concise (avec les groupes formels) se trouve dans 16,17 .

Nous allons tout d'abord ${ }^{10}$ utiliser les formules d'additions sur $\mathbf{G}$ (relatives au plongement $\Phi$ ) et effectuer une translation pour nous ramener à $m=0$. La nullité des dérivées d'ordre $<\underline{t}$ de $F$ au point $m \mathbf{u}$ implique, en utilisant la formule de Leibniz,

(46)

$$
\begin{aligned}
& \frac{1}{\prod_{j=0}^{n}\left(\varphi_{\tau_{j}}^{(j)}\left(m \mathbf{u}_{j}\right)\right)^{D_{j}}} \frac{1}{t !} \mathcal{D} \frac{\underline{\underline{\mathbf{e}}}}{\mathrm{e}} F(m \mathbf{u}) \\
& =\frac{1}{\underline{t} !} \mathcal{D} \underline{\underline{\mathbf{e}}}\left(P \circ\left(\psi_{\tau_{0}}^{(0)}, \ldots, \psi_{\tau_{n}}^{(n)}\right)(z+m \mathbf{u})\right)(0) \\
& \left.=\frac{1}{\underline{t !}} \mathcal{D} \underline{\underline{\mathbf{e}}}\left(P \circ\left(\left(\frac{A_{0}^{(h)}}{A_{\tau_{h}}^{(h)}}\left(\varphi^{(h)}(z), \varphi^{(h)}\left(m \mathbf{u}_{h}\right)\right), \ldots, \frac{A_{N_{h}}^{(h)}}{A_{\tau_{h}}^{(h)}}\left(\varphi^{(h)}(z), \varphi^{(h)}\left(m \mathbf{u}_{h}\right)\right)\right)\right)\right){ }_{h}\right)(0) \\
& =\left\{\prod_{h=0}^{n} \frac{1}{A_{\tau_{h}}^{(h)}\left(\psi_{0}^{(h)}(0), \psi_{\tau_{h}}^{(h)}\left(m \mathbf{u}_{h}\right)\right)^{D_{h}}}\right\} \times \frac{1}{\underline{t !}} \mathcal{D} \underline{\underline{\mathbf{e}}}\left(Q\left(\psi_{0}^{(0)}, \ldots, \psi_{0}^{(n)}\right)\right)(0)
\end{aligned}
$$

\footnotetext{
${ }^{10}$ Dans la littérature, cette première étape porte le nom d'ii astuce d'ANDERSON-BAKER-

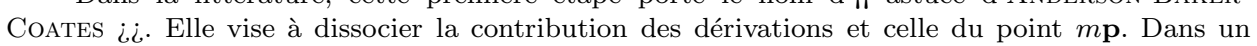
formalisme géométrique plus adéquat ( $c f$. [18]), elle est implicite et n'apparaît plus comme une ii astuce $i$. .
} 
où, si nous posons $\mathbf{X}_{h}:=\left(X_{0}^{(h)}, \ldots, X_{N_{h}}^{(h)}\right)$,

$$
Q\left(\mathbf{X}_{0}, \ldots, \mathbf{X}_{n}\right):=P\left(\left(A_{0}^{(h)}\left(\mathbf{X}_{h}, \psi_{\tau_{h}}^{(h)}\left(m \mathbf{u}_{h}\right)\right), \ldots, A_{N_{h}}^{(h)}\left(\mathbf{X}_{h}, \psi_{\tau_{h}}^{(h)}\left(m \mathbf{u}_{h}\right)\right)\right)_{0 \leq h \leq n}\right) .
$$

Le polynôme $Q$ est homogène en $\mathbf{X}_{h}$ de degré $\mathbf{c}_{h} D_{h}$ où l'on a posé $\mathbf{c}_{h}:=\operatorname{deg}_{\mathbf{X}} A_{0}^{(h)}(\mathbf{X}, \mathbf{Y})$ (cela a un sens par définition des polynômes $A_{0}^{(h)}$, p. 12). Notons $\left(q_{\underline{\lambda}}\right)$ les coefficients du polynôme $Q$.

Soit $\mathcal{F}$ l'ensemble des coefficients de tous les polynômes $A_{j}^{(h)}$ et $C_{k j h}^{(i)}$ (ces polynômes, définis à la fin du $\S$ 司, sont en nombre fini). C'est un sous-ensemble fini de $\mathbf{K}$. Pour toute place ultramétrique $\mathfrak{P}$ de $\mathbf{L}$, le terme $\left|q_{\underline{\lambda}}\right|_{\mathfrak{P}}$ est majoré (uniformément en $\underline{\lambda}$ ) par

(47) $\max _{\underline{\mu}}\left\{\left|p_{\underline{\mu}}\right| \mathfrak{P}\right\} \times \prod_{h=0}^{n} \max \left\{\left|\frac{\varphi_{0}^{(h)}}{\varphi_{\tau_{h}}^{(h)}}\left(m \mathbf{u}_{h}\right)\right|_{\mathfrak{P}}, \ldots,\left|\frac{\varphi_{N_{h}}^{(h)}}{\varphi_{\tau_{h}}^{(h)}}\left(m \mathbf{u}_{h}\right)\right|_{\mathfrak{P}}\right\}^{\mathrm{c}_{h} D_{h}} \times|\mathcal{F}|_{\mathfrak{P}}^{D_{0}+\cdots+D_{n}}$

où $|\mathcal{F}|_{\mathfrak{P}}:=\max _{x \in \mathcal{F}}\left\{1,|x|_{\mathfrak{P}}\right\}$.

Par ailleurs, pour tout $d$-uplet $\underline{h}$ de longueur $<|\underline{t}|$, le terme $\mathcal{D} \frac{h}{\tilde{e}} Q \circ\left(\psi_{0}^{(0)}, \ldots, \psi_{0}^{(n)}\right)(0)$ est nul car cette propriété équivaut, pour tout $\underline{h}$ de longueur $<|\underline{t}|$, à $\mathcal{D} \frac{h}{\tilde{\mathrm{e}}} F(m \mathbf{u})=0$, assertion vraie par définition de $\underline{t}$. Par conséquent, le terme

$$
\underline{\underline{t}} \underline{\underline{1}} \mathcal{D} \underline{\underline{\underline{e}}}\left(Q\left(\psi_{0}^{(0)}, \ldots, \psi_{0}^{(n)}\right)\right)(0)
$$

est le premier (pour l'ordre lexicographique sur $\mathbb{N}^{d+1}$ ) coefficient de Taylor a priori non nul de la fonction holomorphe (au voisinage de 0 )

$$
\begin{aligned}
& Q\left(\psi_{0}^{(0)}, \ldots, \psi_{0}^{(n)}\right)\left(z_{1} \widetilde{e}_{1}+\cdots+z_{d} \widetilde{e}_{d}\right) \\
& =Q\left(1, \beta_{1} z_{1}+\cdots+\beta_{d} z_{d}, \psi_{0}^{(1)}(\mathbf{z}), \ldots, \psi_{0}^{(n)}(\mathbf{z})\right)
\end{aligned}
$$

où $\mathbf{z}=z_{1} e_{1}+\cdots+z_{d} e_{d}$.

Nous allons maintenant effectuer un changement de variables $\left(z_{i}\right)_{i} \rightsquigarrow\left(\mathfrak{t}_{i}\right)_{i}$ et réinterpréter le terme (48) comme le coefficient d'une série en les $\mathfrak{t}_{i}$. Auparavant, nous définissons les paramètres locaux $\mathfrak{t}_{0}, \ldots, \mathfrak{t}_{d}$ de la manière suivante. Posons $\mathfrak{t}_{0}:=z_{0}$ et, pour $i \in\{1, \ldots, n\}$ et $h \in\left\{0, \ldots, \delta_{i}-1\right\}$,

$$
\mathfrak{t}_{\delta_{0}+\cdots+\delta_{i-1}+h}=\frac{\varphi_{h+1}^{(i)}}{\varphi_{0}^{(i)}}
$$

Pour chaque $i \in\{0, \ldots, n\}$, au voisinage de la section nulle de $\mathbf{G}_{i}$, le groupe algébrique $\mathbf{G}_{i}$ est une variété affine et, par conséquent, il existe des polynômes $P_{1}^{(i)}, \ldots, P_{N_{i}^{\prime}}^{(i)}$, à coefficients dans $\mathcal{O}_{\mathbf{K}}$, homogènes en $\mathbf{X}_{i}$ tels que $P_{j}^{(i)}\left(\varphi^{(i)}\right)=0$ $\left(1 \leq j \leq N_{i}^{\prime}\right)$. Notons o la $\mathcal{O}_{\mathbf{K}}$-algèbre engendrée par l'inverse des coefficients de tous les polynômes $P_{j}^{(i)}\left(1 \leq j \leq N_{i}^{\prime}, 0 \leq i \leq n\right)$. Comme $\mathbf{G}_{i}$ est non-singulier (lisse) au voisinage de 0 , nous pouvons choisir $N_{i}^{\prime}=N_{i}-\delta_{i}$ et les polynômes $P_{j}^{(i)}$ $\left(1 \leq j \leq N_{i}^{\prime}\right)$ de telle façon qu'en appliquant le théorème des fonctions implicites, chacune des fonctions $\varphi_{h}^{(i)} / \varphi_{0}^{(i)}$, pour $h=0, \ldots, N_{i}$, soit une série entière en les paramètres $\left(\mathfrak{t}_{\delta_{0}+\cdots+\delta_{i-1}+h}\right)_{0 \leq h \leq \delta_{i}-1}$, à coefficients dans $\mathfrak{o}$, i.e.

$$
\left(\psi_{0}^{(1)}, \ldots, \psi_{0}^{(n)}\right) \in\left(\mathfrak{o}\left[\left[\mathfrak{t}_{1}, \ldots, \mathfrak{t}_{d}\right]\right]\right)^{N_{1}+\cdots+N_{n}+n}
$$

(et, bien sûr, $\left.\psi_{0}^{(0)}=\left(1, \mathfrak{t}_{0}\right)\right)$. En particulier,

$$
Q\left(\psi_{0}^{(0)}, \ldots, \psi_{0}^{(n)}\right)=\sum_{\underline{\alpha} \in \mathbb{N}^{d+1}} \theta_{\underline{\alpha}} \mathfrak{t}_{0}^{\alpha_{0}} \mathfrak{t}_{1}^{\alpha_{1}} \cdots \mathfrak{t}_{d}^{\alpha_{d}}
$$

où $\theta_{\underline{\alpha}}$ est une combinaison linéaire dans o o $[\mathcal{F}]$ des coefficients $q_{\underline{\lambda}}$ de $Q$. De plus $\theta_{\underline{\alpha}}=0$ si $\alpha_{0}>\operatorname{deg}_{\mathbf{X}_{0}} Q=D_{0}$ ). 
Par ailleurs, on notera que (grâce à la normalisation (11) de la jacobienne, p. 13), pour $0 \leq i \leq d$, on a $\mathfrak{t}_{i}=z_{i}+$ termes d'ordres $\geq 2$ en $z_{1}, \ldots, z_{d}$, ce qui implique que $z_{i}=\mathfrak{t}_{i}+$ termes d'ordres $\geq 2$ en $\mathfrak{t}_{1}, \ldots, \mathfrak{t}_{d}$. De plus, d'après l'équation (14), appliquée à chacune des fonctions $\varphi^{(i)}$, on a

$$
\left(\begin{array}{c}
\mathrm{dt}_{1} \\
\vdots \\
\vdots \\
\mathrm{dt}_{d}
\end{array}\right)=\left(\begin{array}{cccc}
1+\star & \star & \cdots & \star \\
\star & 1+\star & \ddots & \vdots \\
\vdots & \ddots & \ddots & \star \\
\star & \ldots & \star & 1+\star
\end{array}\right)\left(\begin{array}{c}
\mathrm{d} z_{1} \\
\vdots \\
\vdots \\
\mathrm{d} z_{d}
\end{array}\right)
$$

où chaque $\star$ représente un élément de $\mathfrak{o}[\mathfrak{F}]\left[\mathfrak{t}_{1}, \ldots, \mathfrak{t}_{d}\right]$, qui s'annule en $(0, \ldots, 0)$ $(\mathfrak{o}[\mathcal{F}]$ est l'algèbre engendrée sur $\mathfrak{o}$ par les éléments de $\mathcal{F})$. Par conséquent, des formules d'inversions de Cramer on déduit

$$
\mathrm{d} z_{i} \in \underset{h=1}{\oplus} \mathfrak{o}[\mathcal{F}]\left[\left[\mathfrak{t}_{1}, \ldots, \mathfrak{t}_{d}\right]\right] \mathrm{d} \mathfrak{t}_{h} .
$$

Ce résultat signifie que, pour $i \in\{1, \ldots, d\}$, on a

$$
z_{i}=\ell_{i}\left(\mathfrak{t}_{1}, \ldots, \mathfrak{t}_{d}\right) \quad \text { où } \quad \ell_{i}=\sum_{\underline{n} \in \mathbb{N}^{d}} a_{\underline{n}}^{(i)} \mathfrak{t}_{1}^{n_{1}} \cdots \mathfrak{t}_{d}^{n_{d}}
$$

est une série formelle (appelée logarithme formel) dont les coefficients $a_{n}^{(i)}$ sont une somme de termes de la forme $b / m$, avec $b \in \mathfrak{o}[\mathcal{F}]$ et $m$ est égal à une des composantes du $d$-uplet $\underline{n}$. Remplaçons alors dans (49) les $z_{i}$ par leur expression ci-dessus en fonction des $\mathfrak{t}_{i}$. Compte tenu de $z_{i}=\mathfrak{t}_{i}+$ termes d'ordres $\geq 2$, on en déduit que le terme (48) est aussi le coefficient de Taylor d'ordre $\underline{t}$ de la série

$$
\sum_{\substack{\alpha \in \mathbb{N}^{\alpha}+1 \\ \alpha_{0} \leq D_{0}}} \theta_{\underline{\alpha}}\left(\beta_{1} \ell_{1}+\cdots+\beta_{d} \ell_{d}\right)^{\alpha_{0}} \mathfrak{t}_{1}^{\alpha_{1}} \cdots \mathfrak{t}_{d}^{\alpha_{d}} .
$$

Posons $\gamma_{\underline{n}}:=\sum_{i=1}^{d} \beta_{i} a_{\underline{n}}^{(i)}$. Alors le coefficient devant $\mathfrak{t}_{1}^{t_{1}} \cdots \mathfrak{t}_{d}^{t_{d}}$ de $(52)$ est une somme de termes de la forme:

$$
\theta_{\underline{\alpha}} \gamma_{\underline{n}_{1}} \cdots \gamma_{\underline{n}_{\alpha_{0}}} .
$$

Compte tenu de la nature arithmétique des coefficients des $\ell_{i}$, rappelée ci-dessus, chacune des quantités (53) est elle-même une somme de termes de la forme $b^{\prime} /\left(m_{1} \ldots m_{\alpha_{0}}\right)$, où $b^{\prime} \in \mathfrak{o}[\mathcal{F}]$ et $m_{1}, \ldots, m_{\alpha_{0}}$ sont des entiers non nuls, dont la somme est $\leq|\underline{n}|$. Par conséquent, la quantité

$$
\delta_{|\underline{\underline{t}}|}\left(D_{0}\right) \theta_{\underline{\alpha}} \gamma_{\underline{n}_{1}} \ldots \gamma_{\underline{n}_{\alpha_{0}}}
$$

(pour $\underline{n}_{1}+\cdots+\underline{n}_{\alpha_{0}} \leq \underline{t}$, au sens lexicographique) est un polynôme en $\beta_{1}, \ldots, \beta_{d}$, à coefficients dans $\sum_{\underline{\lambda}} q_{\underline{\lambda}} \cdot \mathfrak{o}[\mathcal{F}]$, de degré $\leq D_{0}$. Notons $\mathcal{S}$ l'ensemble des places ultramétriques $\mathfrak{P}$ de $\mathbf{L}$ telles que $\mathfrak{o}[\mathcal{F}] \otimes \mathcal{O}_{\mathbf{L}_{\mathfrak{P}}} \neq \mathcal{O}_{\mathbf{L}_{\mathfrak{P}}}$. L'estimation $\mathfrak{P}$-adique, pour $\mathfrak{P} \in M_{\mathbf{L}, f} \backslash \mathcal{S}$, du coefficient de Taylor

$$
\frac{1}{\underline{t} !} \mathcal{D} \underline{\underline{\mathbf{e}}} Q\left(\psi_{0}^{(0)}, \ldots, \psi_{0}^{(n)}\right)(0)
$$

est alors immédiate :

$$
\begin{aligned}
& \left|\underline{\frac{1}{t} !} \mathcal{D} \frac{\underline{t}}{\tilde{\mathbf{e}}} Q\left(\psi_{0}^{(0)}, \ldots, \psi_{0}^{(n)}\right)(0)\right|_{\mathfrak{P}} \\
& \quad \leq\left|\frac{1}{\delta_{|\underline{t}|}\left(D_{0}\right)}\right|_{\mathfrak{P}} \max \left\{1,\left|\beta_{1}\right|_{\mathfrak{P}}, \ldots,\left|\beta_{d}\right|_{\mathfrak{P}}\right\}^{D_{0}} \max _{\underline{\lambda}}\left\{\left|q_{\underline{\lambda}}\right|_{\mathfrak{P}}\right\},
\end{aligned}
$$

majoration de laquelle se déduit (45) via l'égalité (46) et la majoration (47) des coefficients $q_{\underline{\lambda}}$. 
Si $\mathfrak{P} \in \mathcal{S}$, le procédé ci-dessus ne permet pas d'obtenir directement une majoration (même plus faible) de (54) car on ne connaît pas précisément la dépendance en les éléments de o $[\mathcal{F}]$ de ce terme. Dans ce cas, nous disposons cependant d'une majoration similaire légèrement plus faible :

Lemme 6.34. Avec les notations du lemme précédent, il existe une constante $c_{38}$ ne dépendant que de $(\mathbf{G}, \Phi, \mathbf{e})$, telle que, pour toute place $\mathfrak{P} \in \mathcal{S}$, on ait :

$$
\begin{aligned}
& \left|\frac{1}{\prod_{j=0}^{n}\left(\varphi_{\tau_{j}}^{(j)}\left(m \mathbf{u}_{j}\right)\right)^{D_{j}}} \frac{1}{\underline{t} !} \mathcal{D} \underset{\widetilde{\mathbf{e}}}{\underline{t}} F(m \mathbf{u})\right|_{\mathfrak{P}} \\
& \leq c_{38}^{T} \times \max \left\{1,\left|\beta_{1}\right|_{\mathfrak{P}}, \ldots,\left|\beta_{d}\right|_{\mathfrak{P}}\right\}^{D_{0}} \\
& \times \max _{\underline{\lambda}}\left\{\left|p_{\underline{\lambda}}\right| \mathfrak{P}\right\} \times \prod_{h=0}^{n} \frac{1}{\left|A_{\tau_{h}}^{(h)}\left(\psi_{0}^{(h)}(0), \psi_{\tau_{h}}^{(h)}\left(m \mathbf{u}_{h}\right)\right)\right|_{\mathfrak{P}}^{D_{h}}} \\
& \times \prod_{j=0}^{n} \max \left\{\left|\frac{\varphi_{0}^{(j)}\left(m \mathbf{u}_{j}\right)}{\varphi_{\tau_{j}}^{(j)}\left(m \mathbf{u}_{j}\right)}\right|_{\mathfrak{P}}, \cdots,\left|\frac{\varphi_{N_{j}}^{(j)}\left(m \mathbf{u}_{j}\right)}{\varphi_{\tau_{j}}^{(j)}\left(m \mathbf{u}_{j}\right)}\right|_{\mathfrak{P}}\right\}^{\mathbf{c}_{j} D_{j}} .
\end{aligned}
$$

Démonstration. Pour contourner le problème évoqué en préambule du lemme, nous allons majorer ii trivialement i.ं, ce coefficient grâce à la propriété de différentiabilité des fonctions $\varphi_{j}^{(h)} / \varphi_{0}^{(h)}(c f . \S$ 5). Reprenons les notations introduites dans la preuve précédente. Il existe un polynôme $C_{k j}$ ne dépendant que de $\mathbf{G}$ tel que

$$
\frac{\partial}{\partial z_{k}}\left(\frac{\varphi_{j}^{(h)}}{\varphi_{0}^{(h)}}\right)=C_{k j}\left(\psi_{0}^{(0)}, \ldots, \psi_{0}^{(n)}\right)
$$

Nous développons

$$
\mathcal{D} \overline{\tilde{\mathbf{e}}}=\sum_{0 \leq m_{i} \leq t_{i}}\left(\prod_{i=1}^{d}\left(\begin{array}{c}
t_{i} \\
m_{i}
\end{array}\right) \cdot \beta_{i}^{m_{i}} \cdot\left(\frac{\partial}{\partial z_{i}}\right)^{t_{i}-m_{i}}\right)\left(\frac{\partial}{\partial z_{0}}\right)^{m_{1}+\cdots+m_{d}}
$$

Appliquée à $Q\left(\psi_{0}^{(0)}, \ldots, \psi_{0}^{(n)}\right)$, la somme se restreint à $m_{1}+\cdots+m_{d} \leq D_{0}$ car la fonction $Q\left(\psi_{0}^{(0)}, \ldots, \psi_{0}^{(n)}\right)$ est polynomiale en la variable $z_{0}$ de degré $\leq D_{0}$. Utilisons récursivement les égalités du type (56); il vient :

$$
\begin{aligned}
\left|\mathcal{D} \frac{\underline{\underline{e}}}{\underline{\mathbf{e}}} Q\left(\psi_{0}^{(0)}, \ldots, \psi_{0}^{(n)}\right)(0)\right|_{\mathfrak{P}} \leq|\mathcal{F}|_{\mathfrak{P}}^{|\underline{t}|+D_{0}+\cdots+D_{n}} \times \max _{\underline{\lambda}}\left\{\left|q_{\underline{\lambda}}\right|_{\mathfrak{P}}\right\} \\
\times \max \left\{1,\left|\beta_{1}\right|_{\mathfrak{P}}, \ldots,\left|\beta_{d}\right|_{\mathfrak{P}}\right\}^{D_{0}} .
\end{aligned}
$$

Compte tenu de l'évaluation $\mathfrak{P}$-adique de $\frac{1}{t !}$ (une exponentielle de $\left.|\underline{t}|\right)$, on obtient le résultat annoncé, grâce au majorant $(47)$.

\subsection{Estimations archimédiennes.}

Lemme 6.35. Avec les notations du lemme 6.33, il existe une constante $c_{39}$ ne dépendant que de $(\mathbf{G}, \Phi, \mathbf{e})$ telle que, pour toute place archimédienne $\sigma: \mathbf{L} \rightarrow \mathbb{C}$, 
on ait

$$
\begin{aligned}
& \left|\frac{1}{\prod_{j=0}^{n}\left(\varphi_{\tau_{j}}^{(j)}\left(m \mathbf{u}_{j}\right)\right)^{D_{j}}} \frac{1}{\underline{t} !} \mathcal{D} \frac{\underline{\underline{e}}}{\tilde{\mathbf{e}}} F(m \mathbf{u})\right|_{\sigma} \\
& \leq c_{39}^{T} \times \max \left\{1,\left|\beta_{1}\right|_{\sigma}, \ldots,\left|\beta_{d}\right|_{\sigma}\right\}^{D_{0}} \times \max _{\underline{\underline{\lambda}}}\left\{\left|p_{\underline{\lambda}}\right|_{\sigma}\right\} \\
& \quad \times \prod_{h=0}^{n} \frac{1}{\left|A_{\tau_{h}}^{(h)}\left(\psi_{0}^{(h)}(0), \psi_{\tau_{h}}^{(h)}\left(m \mathbf{u}_{h}\right)\right)\right|_{\sigma}^{D_{h}}} \\
& \quad \times \prod_{j=0}^{n} \max \left\{\left|\frac{\varphi_{0}^{(j)}\left(m \mathbf{u}_{j}\right)}{\varphi_{\tau_{j}}^{(j)}\left(m \mathbf{u}_{j}\right)}\right|_{\sigma}, \cdots,\left|\frac{\varphi_{N_{j}}^{(j)}\left(m \mathbf{u}_{j}\right)}{\varphi_{\tau_{j}}^{(j)}\left(m \mathbf{u}_{j}\right)}\right|_{\sigma}\right\}^{\mathbf{c}_{j} D_{j}} .
\end{aligned}
$$

Démonstration. C'est exactement la même démonstration que celle du paragraphe précédent pour les places $\mathfrak{P} \in \mathcal{S}$. La constante $c_{39}$ n'est pas tout à fait $\max _{\sigma}\left\{|\mathfrak{F}|_{\sigma}\right\}^{n+2}$ car, contrairement au cas ultramétrique où la valeur absolue d'une somme est majorée par le maximum des termes de la somme, nous devons tenir compte de la longueur des polynômes $A_{\tau_{h}}^{(h)}$ et $C_{k j}$ (notamment dans la majoration de $\left.\left|q_{\underline{\lambda}}\right|_{\sigma}\right)$.

6.13. Conclusion. Des estimations $\mathfrak{P}$-adiques et archimédiennes du nombre algébrique

$$
\frac{1}{\prod_{j=0}^{n}\left(\varphi_{\tau_{j}}^{(j)}\left(m \mathbf{u}_{j}\right)\right)^{D_{j}}} \frac{1}{t !} \mathcal{D} \frac{t}{\tilde{\mathbf{e}}} F(m \mathbf{u})
$$

nous allons en déduire une minoration de $\left|\frac{1}{\underline{\underline{t}}} \mathcal{D} \underset{\widetilde{\mathbf{e}}}{\underline{t}} F(m \mathbf{u})\right|$. La comparaison avec la majoration de ce nombre complexe obtenue à la suite de l'extrapolation (corollaire 6.28) donnera une contradiction. Nous en déduirons alors les théorèmes 3.1 et 3.2 .

Appliquons la formule du produit au nombre (58). Nous obtenons l'inégalité suivante :

$$
\begin{aligned}
& \frac{1}{D} \log \left|\frac{1}{\underline{t} !} \mathcal{D} \underline{\underline{\underline{e}}} F(m \mathbf{u})\right| \geq \\
& -\left(c_{40} T+D_{0} h(W)+h(P)+\log \delta_{|\underline{t \mid}|}\left(D_{0}\right)\right. \\
& \quad+\frac{1}{D} \log \prod_{h=0}^{n}\left|A_{\tau_{h}}^{(h)}\left(\psi_{0}^{(h)}(0), \psi_{\tau_{h}}^{(h)}\left(m \mathbf{u}_{h}\right)\right)\right|^{D_{h}} \\
& \left.\quad+\sum_{j=0}^{n} \mathbf{c}_{j} D_{j}\left\{\frac{1}{D} \sum_{v \neq \sigma_{0}} \log \max \left\{\left|\frac{\varphi_{0}^{(j)}(m \mathbf{u})}{\varphi_{\tau_{j}}^{(j)}(m \mathbf{u})}\right|_{v}, \cdots,\left|\frac{\varphi_{N_{j}}^{(j)}(m \mathbf{u})}{\varphi_{\tau_{j}}^{(j)}(m \mathbf{u})}\right|_{v}\right\}\right\}\right)
\end{aligned}
$$

(la dernière somme porte sur toutes les places (finies et infinies) de $\mathbf{L}$, différentes de $\left.\sigma_{0}\right)$. La définition des polynômes $A_{\tau_{h}}^{(h)}$ implique l'existence d'une constante $c_{41}$, ne dépendant que de $(\mathbf{G}, \Phi, \mathbf{e})$, telle que, pour tout $h \in\{0, \ldots, n\}$,

$$
\left|A_{\tau_{h}}^{(h)}\left(\psi_{0}^{(h)}(0), \psi_{\tau_{h}}^{(h)}\left(m \mathbf{u}_{h}\right)\right)\right| \leq c_{41} \max \left\{\left|\frac{\varphi_{0}^{(h)}(m \mathbf{u})}{\varphi_{\tau_{h}}^{(h)}(m \mathbf{u})}\right|, \cdots,\left|\frac{\varphi_{N_{h}}^{(h)}(m \mathbf{u})}{\varphi_{\tau_{h}}^{(h)}(m \mathbf{u})}\right|\right\}^{\mathbf{c}_{h}} .
$$

Le minorant de l'inégalité (59) se simplifie alors et il peut être remplacé par

$$
-c_{42}\left\{T \log D_{0}+D_{0} h(W)+h(P)+\sum_{j=0}^{n} D_{j} h\left(m \mathbf{p}_{j}\right)\right\} \text {. }
$$


Choisissons un $n$-uplet $\left(\tau_{1}, \ldots, \tau_{n}\right)$ tel que

$$
\begin{aligned}
\left|\varphi_{\tau_{j}}^{(j)}(m \mathbf{u})\right| & =\max \left\{\left|\varphi_{0}^{(j)}(m \mathbf{u})\right|, \ldots,\left|\varphi_{N_{j}}^{(j)}(m \mathbf{u})\right|\right\} \\
& \geq \exp \left\{-c_{j}^{-}\left(1+m\left\|\mathbf{u}_{j}\right\|\right)^{\rho_{j}}\right\} \text { lorsque } j \neq 0
\end{aligned}
$$

et

$$
\left|\varphi_{\tau_{0}}^{(0)}(m \mathbf{u})\right| \geq 1
$$

La quantité $\log \left|\frac{1}{\underline{t} !} \mathcal{D} \frac{t}{\widetilde{\mathbf{e}}} F(m \mathbf{u})\right|$ est alors minorée par

$$
\begin{aligned}
-c_{43}\{ & D T \log D_{0}+D_{0}\left(D h(W)+\max _{0 \leq s \leq(d+1) S}\left\{D h\left(s u_{0}\right)\right\}\right) \\
& \left.+D h(P)+\sum_{j=1}^{n} D_{j}\left(\max _{0 \leq s \leq(d+1) S}\left\{D h\left(s \mathbf{p}_{j}\right)\right\}+\left(S\left\|\mathbf{u}_{j}\right\|\right)^{\rho_{j}}\right)\right\} .
\end{aligned}
$$

En remplaçant les paramètres par leur valeur, nous obtenons

$$
\log \left|\frac{1}{\underline{t} !} \mathcal{D} \frac{\underline{t}}{\widetilde{\mathbf{e}}} F(m \mathbf{u})\right| \geq-c_{44} C_{0}^{1 / 2} U_{0} .
$$

Ce qui contredit, pour $C_{0}$ assez grand, la majoration du corollaire 6.28 (p. 29). Ainsi, une des trois hypothèses ${ }^{11}$ émises lors de la démonstration est fausse. Donc

(1) Ou bien l'inégalité (38) est fausse et alors

$$
\log |\Lambda| \geq-C_{0} U_{0} \text {. }
$$

(2) Ou bien $\mathbf{u} \in t_{\widetilde{\mathbf{G}}}(\mathbb{C})$ (et nécessairement nous sommes dans le cas périodique). Le théorème se déduit alors du lemme 6.8.

(3) Ou bien $\mathbf{u} \notin t_{\widetilde{\mathbf{G}}}(\mathbb{C})$ et nous sommes dans le cas périodique. Alors

$$
|\Lambda| \geq \frac{1}{2} \mathrm{~d}\left(\mathbf{u}, t_{\widetilde{\mathbf{G}}}(\mathbb{C})\right),
$$

et d'après la première partie du corollaire 6.9, le logarithme de $2|\Lambda|$ est minoré par le logarithme de

$$
C_{0}^{-23 d}\left(D+D h\left(\mathbf{p}_{1}\right)+\cdots+D h\left(\mathbf{p}_{n}\right)+E\left\|\mathbf{u}_{1}\right\|+\cdots+E\left\|\mathbf{u}_{n}\right\|\right)^{-20 d}
$$

et donc par $-U_{0}$.

Pour obtenir le théorème 3.1, il ne reste plus qu'à remplacer dans $U_{0}$, l'entier $S$ par $\mathfrak{a}$, la comparaison entre $|\Lambda|$ et $\mathrm{d}(\mathbf{u}, W)$ s'effectuant via l'égalité (3). Quant au théorème 3.2, les hypothèses de ce théorème impliquent que nous sommes nécessairement dans le cas non-périodique. Tous les calculs effectués dans ce cas restent vrais lorsque $U_{0}$ est remplacé par $U_{0}^{\prime}$. La seule modification notable se situe au niveau du lemme de zéros, en particulier lors du corollaire 6.19, p. 23, où nous invitons le lecteur à se reporter pour de plus amples détails.

\section{Remerciements.}

Ce travail constitue la première partie de ma thèse de doctorat effectuée sous la direction de G. Diaz, S. David et M. Waldschmidt. Je les remercie vivement de m'avoir proposé ce sujet ainsi que de l'aide et de l'attention qu'ils m'ont apportées au cours de ces années passées ensemble. Leur soutien constant et leurs encouragements m'ont été extrêmement précieux. Je remercie également N. HiRATA-KoHNO d'avoir pris le temps de vérifier les nombreux points techniques de cet article en acceptant d'avoir rapporté ce chapitre de ma thèse. Je remercie J.-B. Bost et M. LAUREnT pour l'intérêt qu'ils ont manifesté pour ce travail ainsi que le rapporteur de cet article pour ses remarques qui m’ont permis d'éclaircir et de préciser

\footnotetext{
${ }^{11}$ Deux de ces hypothèses sont dans le corollaire 6.9 (p. 20), la troisième est au début du $\S 6.10$ (p. 28).
} 
certains passages. Enfin, je remercie chaleureusement toute l'équipe de Théorie des Nombres de Saint-Étienne pour les excellentes conditions de travail qu'ils m'ont offertes durant ma thèse.

\section{RÉFÉRENCES}

[1] M. ABLy. Formes linéaires de logarithmes de points algébriques sur une courbe elliptique de type CM. Ann. Inst. Fourier Grenoble, 50(1):1-33, 2000.

[2] M. Ably et É. Gaudron. Approximation diophantienne sur les courbes elliptiques à multiplication complexe. C. R. Acad. Sci, I 337 :629-634, 2003.

[3] D. Bertrand. Galois representations and transcendental numbers. Dans New advances in Transcendence Theory. Cambridge University Press, 1988.

[4] D. Bertrand. Duality on tori and multiplicative dependence relations. J. Austral. Math. Soc., 62 (Series A) :198-216, 1997.

[5] D. Bertrand et P. Philippon. Sous-groupes algébriques de groupes algébriques commutatifs. Illinois J. Math, $23: 263-280,1988$.

[6] E. Bombieri et J. VaAler. On Siegel's lemma. Invent. Math., 73 :11-32, 1983. Addendum 75,377 (1984).

[7] J.-B. Bost. Périodes et isogénies des variétés abéliennes sur les corps de nombres (d'après D. Masser et G. Wüstholz). Séminaire Bourbaki, volume 237 de Astérisque, pages 115-161. Société Mathématique de France, 1996.

[8] S. BRulltet. D'une mesure d'approximation simultanée à une mesure d'irrationalité : le cas de $\Gamma(1 / 4)$ et $\Gamma(1 / 3)$. Acta Arith., 104 (3) :243-281, 2002.

[9] D.V. Chudnovsky et G.V. Chudnovsky. Padé approximations and diophantine geometry. Dans Proc. Natl. Acad. Sci. USA, volume 82, pages 2212-2216, 1985.

[10] G.V. Chudnovsky. Measures of irrationality, transcendence and algebraic independence. Dans Recent progress in Number Theory, pages 11-82. Cambridge Univ. Press., 1980.

[11] G.V. Chudnovsky. Contributions to the theory of transcendental numbers, volume XI. Providence, R.I. : American Math. Soc., 1984. Mathematical Surveys and Monographs.

[12] S. DAvid. Minorations de formes linéaires de logarithmes elliptiques, volume 62 de Mémoire de la Société Mathématique de France. 1995.

[13] S. DAvid et N. HiRATA-Kohno. Recent progress on linear forms in elliptic logarithms. Dans A Panorama in Number Theory, chap. 3. Cambridge Univ. Press., 2002. G. Wüstholz (Éd).

[14] S. David et N. Hirata-Kohno. Linear forms in elliptic logarithms. En préparation, 2002.

[15] G. Diaz. Minorations de combinaisons linéaires non homogènes pour un logarithme elliptique. C. R. Acad. Sci, I 318(10):879-883, 1994. ii Tapuscrit ii de la démonstration détaillée.

[16] É. Gaudron. Mesure d'indépendance linéaire de logarithmes dans un groupe algébrique commutatif. C. R. Acad. Sci, I 333 :1059-1064, 2001.

[17] É. Gaudron. Mesure d'indépendance linéaire de logarithmes dans un groupe algébrique commutatif. Thèse de doctorat, Université Jean Monnet de Saint-Étienne, décembre 2001.

[18] É. Gaudron. Formes linéaires de logarithmes effectives sur les variétés abéliennes. Prépublication 2004, 64 pages.

[19] N. Hirata-Kohno. Formes linéaires de logarithmes de points algébriques sur les groupes algébriques. Invent. Math., 104 :401-433, 1991.

[20] N. Hirata-Kohno. Approximations simultanées sur les groupes algébriques commutatifs. Compos. Math., 86 :69-96, 1993.

[21] M. Laurent et D. Roy. Sur l'approximation algébrique en degré de transcendance un. Ann. Inst. Fourier Grenoble, 49(1) :27-55, 1999.

[22] D.W. MASSER. Linear relations on algebraic groups. Dans New advances in transcendence theory (Durham, 1986), pages 248-262, Cambridge Univ. Press, Cambridge, 1988.

[23] P. Philippon. Lemme de zéros dans les groupes algébriques commutatifs. Bull. Soc. Math. France, 114 :355-383, 1986. Errata et Addenda, id.115 (1987).

[24] P. Philippon. Nouveaux lemmes de zéros dans les groupes algébriques commutatifs. Rocky Mt. J. Math., 26(3) :1069-1088, 1996.

[25] P. Philippon et M. WALdschmidt. Formes linéaires de logarithmes sur les groupes algébriques commutatifs. Illinois J. Math., 32(2):281-314, 1988. 
[26] P. Philippon et M. Waldschmidt. Formes linéaires de logarithmes simultanées sur les groupes algébriques commutatifs. Dans Séminaire de Théorie des Nombres Paris 1986-87, volume 75 de Progress in Mathematics, pages 313-347. Birkhäuser Boston, Inc., 1989. Édité par Catherine Goldstein.

[27] W.M. Schmidt. Diophantine Approximations and Diophantine Equations, volume 1467 de Lecture Notes in Math. Springer-Verlag, 1991.

[28] J.-P. SERRE. Quelques propriétés des groupes algébriques commutatifs. Appendice II de [29].

[29] M. Waldschmidt. Nombres transcendants et groupes algébriques, Astérisque, volume 69/70. Société Mathématique de France, 1979.

[30] M. Waldschmidt. A lower bound for linear forms in logarithms. Acta Arith, 37 :257-283, 1980.

[31] M. WAldschmidt. Nouvelles méthodes pour minorer des combinaisons linéaires de logarithmes de nombres algébriques. Séminaire de Théorie des Nombres de Bordeaux, Ser. II 3(1) :129-185, 1991.

[32] M. Waldschmidt. Approximation diophantienne dans les groupes algébriques commutatifs. (I) : Une version effective du théorème du sous-groupe algébrique. J. reine angew. Math, $493: 61-113,1997$.

[33] M. Waldschmidt. Diophantine Approximation On Linear Algebraic Groups, volume 326 de Grundlehren der mathematischen Wissenschaften. Springer, 2000.

[34] G. Wüstholz. Algebraische punkte auf analytischen untergruppen algebraischer gruppen. Annals of Math., 129(3):501-517, 1989. 